\title{
Adaptive Learning for Model Driven Control of Cloud Applications
}

\author{
by
}

Michael Dang'ana

A thesis submitted to the Faculty of Graduate and Postdoctoral Affairs in partial fulfillment of the requirements for the degree of

Master of Applied Science

in

Electrical and Computer Engineering

Carleton University

Ottawa, Ontario

(C)2019

Michael Dang'ana 


\begin{abstract}
System performance tracking, planning and on-demand provisioning are common techniques employed today for management of cloud applications. This work explores the use of machine learning algorithms to tune Kalman filters, which track system performance metrics to make predictions about the future state of running applications. The benefit of accurate predictions is to enable planning algorithms, for example model-based schemes like layered queueing networks, to determine potential system choke points with high granularity and specificity. The focus of this work is to evaluate the impact of long short term neural networks on improving the accuracy of Kalman filter predictions via tuning, to use these predictions to drive layered queueing network model driven decision control actions, and to evaluate the performance of this controller in different virtual machine types in the cloud.

In addition, a new scheme for layered queueing network model parameter tracking using principal component analysis is proposed and evaluated.
\end{abstract}




\section{Acknowledgements}

Thanks to Prof. Murray Woodside for his mentorship and direction in creating and executing this research project. Also, his extensive research on Layered Queueing Networks and other areas of performance engineering have been indispensable in providing the conceptual foundation for courses taken and for the masters program as a whole.

This effort could not have been possible without Performance Engineering courses and guidance, especially due to direction offered by Prof. Dorina Petriu. It was also impossible to attempt this task without Pattern Classification teaching and project advice provided by Prof. James Green.

Lastly, brainstorming sessions and research demonstrations in the RADS lab were engaging and insightful, and provided the ideas leading to selection of this topic and framing of the tasks. For that, many thanks to the members of the lab, and to Prof. Samuel Ajila for excellent tips and stimulating discussions on these and related research topics. 
$\begin{array}{ll}\text { Abstract } & 2\end{array}$

$\begin{array}{ll}\text { Acknowledgements } & 3\end{array}$

$\begin{array}{ll}\text { List of Notations } & 7\end{array}$

List of Appendices 9

List of Figures \& Illustrations 9

Chapter 1: Introduction $\quad 13$

1.1: The Problem and the Tools 16

1.2: Research Questions and Goals 17

1.3: Contributions 19

1.4: Thesis Organization $\quad 20$

Chapter 2: Background $\quad 22$

2.1: Machine Learning For Kalman Filters 22

2.2: Long Short Term Memory Cell [109] 25

2.3: Extended Kalman Filter 27

2.3.1: Measurement Matrix \& Its Jacobian 28

2.3.2: Kalman Filter Error 29

2.4: Kalman Filters For Model Parameter Tracking 29

Chapter 3: Review Of Related Work 32

3.1: Machine Learning \& Kalman Filters 32

3.1.1: Model Extraction From Sequential Data 32

3.1.2: LSTM For Anomaly Detection \& Kalman Filters For State Tracking 34

3.1.3: LSTM \& RNN Training Cost, Loss Functions \& Learning Rates 35

3.1.4: Improving Time Series Prediction Using LSTM 36

3.1.5: Multiple Regression \& Feature Selection 37

3.2: Principal Component Analysis 39

3.3: Autoscaling Of Microservices 41

Chapter 4: PCA Model Tracking Algorithm (KF-PCA) 44

4.1: PCA-LQN Parameter Inference From Kalman Filter Predictions 44

Chapter 5: Project Description $\quad 48$

5.1: Project Organization $\quad 48$

Chapter 6: Control System \& Experimental Testbeds 50 
6.1: Control System Architecture $\quad 50$

6.1: Control System Overview 50

6.1.1: Software Requirements 52

6.1.2: Learning (LSTM) Unit 52

6.1.2.1: LSTM Unit Logistic Regression Encoding \& Decoding 54

6.1.2.2: LSTM Parameters 56

6.1.2.2.1: Bias Selection, Weights, Cost \& Optimization 57

6.1.2.3: KF-LSTM Accuracy \& Error 57

6.1.2.3.1: Calculation of LSTM (KF-LSTM1) Regression Prediction Error $\quad 58$

6.1.2.3.2: Calculation of LSTM (KF-LSTM2) Classification Accuracy $\quad 58$

6.1.2.4: KF-LSTM Data Acquisition - Active Learning 60

6.1.3: EKF Unit $\quad 62$

6.1.3.1: Measured Variables \& Normalization Factors 63

6.1.3.2: State Variables $\quad 64$

6.1.3.3: Process Noise, Measurement Noise \& State Transition Matrices $\quad 64$

6.1.3.3.1: Coefficient Selection For Q, R \& F Matrices 65

6.1.4: Control Unit 66

6.1.4.1: Monitors 67

6.1.4.2: Control Algorithm 69

6.1.5: Configuration File \& Control Commands 70

6.1.5.1: Application Model 73

6.1.5.1.1: Application Model-based Control Behaviour 74

6.2: Control System Testbed $\quad 74$

6.2.1: Test Application Implementation \& Deployment 75

6.2.2: Testbed Client \& Traffic Generators 76

6.3: Performance Model Tracking Testbed $\quad 78$

6.3.1: Testbed Components $\quad 80$

6.3.2: $\mathrm{H}_{\mathrm{k}}$ Matrix Prediction LSTM Model 81

6.3.3: Model Output \& Training Error $\quad 81$

Chapter 7: Experiment Plan $\quad 83$

7.1: Workload Selection \& Characterization 84

7.2: Tuning Experiments $\quad 85$

7.2.1: Arrival Rates 86

7.3: Experimental Factors 87

$\begin{array}{ll}\text { Chapter 8: Analysis } & 90\end{array}$ 
8.1: Resource Usage Data Parsing \& Normalization $\quad 90$

8.2: Tuning Of The LSTM

8.2.1: Tuning of the Regression LSTM Unit (KF-LSTM1) 92

8.2.2: Tuning of Classification LSTM (KF-LSTM2) 93

8.3: KF-LSTM1 Impact on Kalman Filter Prediction Accuracy 94

8.4: KF-LSTM1 Learning of KF Parameters 102

8.4.1: Estimation of EKF Q, R \& F Matrix Coefficients 102

8.4.2; Convergence of EKF Coefficients In Tuning Mode 103

8.5: KF-LSTM1-Based Model Tracking Testbed Analysis 105

8.5.1: Discovery Of KF Measurement Transform Coefficients Using LSTM 106

8.5.1.1: Speed Of Convergence 107

8.5.2: Direct Calculation Of EKF Measurement Transform 107

8.5.3: Evaluating KF-LSTM Tracking Behaviour 108

8.6: Analysis Of KF-PCA-based Model Tracking Behaviour 111

8.6.1.1: Measurement data $\left(\mathrm{z}_{\mathrm{k}}\right) \quad 115$

8.6.1.2: LQN parameters, real $(\mathrm{p})$ and predicted $\left(\mathrm{x}_{\mathrm{k}-1}\right) \quad 117$

8.6.2: Comparison Of KF-PCA-based and Non-KF-PCA Model Tracking 122

8.6.2.1: Measurement data $\left(\mathrm{z}_{\mathrm{k}}\right) \quad 125$

8.6.2.2: LQN parameters, real $(\mathrm{p})$ and predicted $\left(\mathrm{x}_{\mathrm{k}-1}\right)$

8.7: System Testbed Analysis 132

8.7.1: Active \& Passive Monitor Impact 133

Chapter 9: Controller Evaluation 139

9.1: Evaluating Basic Predictive Control With Varying Burst Sizes $\quad 140$

9.2: Evaluating Average Predictive Control With Varying Burst Sizes 143

9.2.1: Note On Active Control Error Bars 146

9.3: Evaluating Average Non-Predictive Control For Varying Burst Size $\quad 147$

9.4: Evaluating Predictive Control By Burst Size For Virtual Machine Types 150

9.4.1: Predictive Control For Virtual Machine Types At Multiple Burst Sizes 153

Chapter 10: Conclusion $\quad 160$

10.1: Threats to Validity 163

10.2: Future Enhancements \& Research Topics 163

$\begin{array}{ll}\text { Appendices } & 165\end{array}$

$\begin{array}{ll}\text { References } & 176\end{array}$ 


\section{List of Notations}

Notation

ATOM:

BR:

$\mathrm{C}_{\mathrm{k}}$ :

$\mathrm{Ct}:$

DevOps:

EKF:

F:

$f(x)$ :

GPU:

g:

$\mathrm{H}_{\mathrm{k}}$

$\mathrm{J}_{\mathrm{f}}$ :

$\mathrm{KF}$ :

KF-LSTM: $\quad$ LSTM-tuned Kalman Filter

KF-LSTM1: $\quad$ KF tuned by LSTM Linear Regression

KF-LSTM2: $\quad$ KF tuned by LSTM Logistic Regression

KF-PCA: Kalman Filter PCA model tracking algorithm

LQN: $\quad$ Layered Queuing Networks [16]

LSTM: $\quad$ Long Short Term Memory [18] Neural Network
Page

42

58

26

48

27

27

28

81

26

27

28

27

57

58

58

44

43

25 


\begin{tabular}{|c|c|c|}
\hline $\mathrm{m}_{\mathrm{LS}}:$ & Least squares-based PCA to LQN factor & 44 \\
\hline $\max :$ & Maximum value & 138 \\
\hline min: & Minimum value & 139 \\
\hline OS: & Operating System & 52 \\
\hline PCA: & Principal Component Analysis & 39 \\
\hline $\mathrm{p}:$ & Tracked LQN model parameter & 74 \\
\hline QoS: & Quality of service control algorithm [106] & 31 \\
\hline Q: & EKF process noise coefficient matrix [4] & 27 \\
\hline R: & EKF measurement noise coefficient matrix [4] & 27 \\
\hline RMSE: & Root Mean Square Error [62] & 38 \\
\hline $\mathrm{v}_{\mathrm{k}}:$ & EKF measurement noise vector [4] & 27 \\
\hline $\mathrm{w}_{\mathrm{k}}$ : & EKF process noise vector [4] & 27 \\
\hline $\mathrm{x}_{\mathrm{k}}$ : & EKF state vector at time step $\mathrm{k}$ [4] & 27 \\
\hline $\mathrm{X}:$ & EKF state vector $[4]$ & 27 \\
\hline $\mathrm{x}_{\mathrm{k}-1}$ : & EKF state vector prior to update [4] & 27 \\
\hline$\widehat{x}_{\mathrm{klk}-1}:$ & normalized EKF state prior at time step $\mathrm{k}$ [4] & 27 \\
\hline $\mathrm{Z}:$ & EKF measurements vector [4] & 27 \\
\hline $\mathrm{z}_{\mathrm{k}}:$ & EKF measurements at time step $\mathrm{k}$ [4] & 27 \\
\hline$\widehat{z}_{\mathrm{k}}:$ & normalized EKF measurements at time step $\mathrm{k}$ [4] & 27 \\
\hline$\mu(m, s):$ & Normal distribution with mean $\mathrm{m}$ and standard deviation $\mathrm{s}$ & 84 \\
\hline$\sigma$, std: & Standard deviation & 106 \\
\hline
\end{tabular}


$\sigma_{w}: \quad$ Standard deviation over data window w 106

$\sigma_{z j}: \quad$ Softmax computation for value $z_{\mathrm{j}}[7]$

\section{List of Appendices}

Appendix A: $\quad$ Background on the Extended Kalman Filter (EKF) 165

A.1: $\quad$ EKF Prediction-based Controller Actions $\quad 166$

$\begin{array}{lll}\text { A.2: } & \text { EKF Observation and Model Summary } & 167\end{array}$

Appendix B: $\quad$ Long Short Term Memory (LSTM) Implementation 169

$\begin{array}{lll}\text { B.1: } & \text { LSTM Initialization } & 169\end{array}$

$\begin{array}{ll}\text { B.2: } & \text { Training \& Testing } \\ & 170\end{array}$

Appendix C: $\quad$ Generative Models For LQN Graphs 171

C.1: $\quad$ Sequential Graph Generation Process For LQN Models $\quad 172$

C.2: $\quad$ Training Graph Generation Models For LQN Graphs $\quad 173$

\section{List of Figures \& Illustrations}

Illustration 2.2: $\quad$ LSTM Unit $\quad 26$

Figure 2.3: $\quad$ Extended Kalman Filter block diagram [5] 27

Figure 4.1.2: $\quad$ Estimation of application state factor from Kalman filter state 46

Figure 4.1.3: $\quad$ Prediction of LQN parameters from Kalman filter state $\quad 47$

Figure 6.1.1: $\quad$ Control System Architecture $\quad 50$

Table 6.1.1: $\quad$ Output of Linux Top Command 51

Figure 6.1.2.2: $\quad$ Linear Regression LSTM-cell static RNN 56

Illustration 6.1.2.4: Three main active learning scenarios [3] 60 
Figure 6.1.2.5: Active Learning Algorithm for Bootstrapping LSTM Labels 62

Figure 6.1.3.1: Measurement Normalization Factors 63

Figure 6.1.4.1: Host Monitors, Application \& Resource Control 67

Illustration 6.1.5: Sample Application Configuration YAML File 72

Figure 6.1.5: $\quad$ LQN Model of Application \& Resource Components 73

$\begin{array}{lll}\text { Figure 6.2.0: } & \text { Testbed System Layout } & 75\end{array}$

Figure 6.2.2: $\quad$ Test Client Operation Sequence Diagram 77

Figure 6.3.0.1: Model Tracking Testbed Data Flow 79

Table 7.3.0.1: $\quad$ Component Test Scenarios $\quad 88$

Table 7.3.0.2: $\quad$ Controller Evaluation Test Scenarios 89

Figure 8.1: $\quad$ Normalized Process Resource Utilization Over Time 91

Figure 8.2.1: $\quad$ MSE (Mean Square Error) over multiple training epochs 93

Figure 8.2.2: $\quad$ Logistic classification accuracy by epoch (increasing mean) 94

Figure 8.3.0.0: $\quad$ EKF predictions for $\mathrm{z}_{\mathrm{k}}=1.01^{\mathrm{k}} \quad 96$

Figure 8.3.0.1: $\quad$ EKF prediction accuracy for $z_{k}=1.01^{k}$

Figure 8.3.0.4: $\quad$ Raw \& Tuned EKF predictions for $\mathrm{z}_{\mathrm{k}}=\exp (\mathrm{k}) \bmod 50$ (18) 98

Figure 8.3.0.5: Raw \& Tuned EKF accuracies for $\mathrm{z}_{\mathrm{k}}=\mathrm{u}(0,1) \quad 98$

Figure 8.3.0.6: Raw \& Tuned EKF predictions for $\mathrm{z}_{\mathrm{k}}=\mathrm{u}(0,1)(19)$

Figure 8.3.0.7 EKF Raw \& Tuned accuracies for $\mathrm{z}_{\mathrm{k}}=\sin (\mathrm{k})+0.3 \mathrm{u}(0,1)$

Figure 8.3.0.8: $\quad$ Predicted priors for raw and tuned EKFs tracking $z_{k}=\sin (k) \quad 100$

Figure 8.3.0.9: Raw \& Tuned EKF accuracies for $z_{k}=0.3 u(0,1)(20)$

Figure 8.3.0.10: Raw and Tuned EKF accuracies for $\mathrm{z}_{\mathrm{k}}=\operatorname{step}(\mathrm{k})(21) \quad 100$ 
Figure 8.3.0.11: Raw \& Tuned EKF predictions for eq (21)

Table 8.4.1.1: Discovered F-diagonal coefficients for real and simulated

Figure 8.4: Aggregate coefficients, individual coefficients \& gradients

Figure 8.5.1: $\quad \mathrm{H}_{\mathrm{k}}$ Matrix LSTM Training Error By Epoch

Figure 8.5.2: $\quad$ Model Tracking For LQN Model Parameter (\# of users = 7)

Figure 8.5.3: $\quad$ Model Tracking For LQN Model Parameter (\# of users = 12)

Figure 8.6.0.1: LQN Parameter, measurements KF Prior \& Error - Iteration 1

Figure 8.6.0.2: $\quad$ LQN Parameter, measurements KF Prior \& Error - Iteration 2

Figure 8.6.0.3: LQN Parameter, measurements KF Prior \& Error - Iteration 3

Figure 8.6.0.5: Summary LQN Parameter Error Percentages Over Iterations

Figure 8.6.1.0: $\quad$ Non PCA LQN Parameters \& KF Prior \& Error - Iteration $1 \quad 123$

Figure 8.6.1.1: Non PCA LQN Parameters \& KF Prior \& Error - Iteration 2123

Figure 8.6.1.2: $\quad$ Non PCA LQN Parameters \& KF Prior \& Error - Iteration 3124

Figure 8.6.1.4: $\quad$ Summary Of Non-PCA LQN Error Percentages

Figure 8.7.1.1: Passive Control Client Requests Over Time

Figure 8.7.1.2: Active Control Client Requests Over Time

Figure 8.7.1.3: Passive monitor metrics for testbed application host

Figure 8.7.1.4: Active monitor metrics for testbed application host

Figure 9.1.1: $\quad$ CPU Time for various burst sizes in predictive control

Figure 9.1.2: $\quad \%$ Memory usage for various burst sizes in predictive control 
Figure 9.2.2: $\quad$ Shared memory for various burst sizes in predictive control 145

Figure 9.2.3: (24) Metrics for top 3 processes sorted by CPU usage 146

Figure 9.3.1: $\quad$ System metrics in basic mode for bust rate (3) 148

Figure 9.3.2: (24) Metrics for top 3 processes sorted by CPU usage 149

Table 9.4: $\quad$ Amazon Web Services EC2 Instance Types $\quad 151$

Figure 9.4.0.1: CPU time, \% memory and shared memory by machine type 152

Figure 9.4.0.2: $\quad$ CPU time by machine type (minus m4.large for readability) 153

Figure 9.4.1: $\quad$ Metric Values By VM Type For Burst Size (3) 154

Figure 9.4.2: $\quad$ Normalized Metric Values By VM Type For Burst Size (10) 155

Figure 9.4.3: $\quad$ Normalized Metric Values By VM Type For Bust Size (20) 156

Figure 9.4.4: $\quad$ CPU Usage For Increasing Burst Rates $\quad 158$ 


\section{Chapter 1: Introduction}

Running applications can be viewed as time varying systems. A system can be described as a set of objects which are interrelated, and are delineated by some kind of spatial or temporal boundary. Time varying systems are systems whose parameters change over time based on predefined laws or external factors like time-delayed or time-advanced inputs. Controlling time varying systems is a problem that has been tackled by various algorithms, with adaptive control specifically designed for this purpose. One of the main issues with time varying systems is changing process characteristics, that create a need for the controller to recognize these changes and retune itself [93]. Techniques that have been applied to this type of problem include higher order control, fuzzy logic, stochastic approximation, machine learning, often algorithms that model non-linear system parameters. Machine learning algorithms are quite suitable in being able to model such systems for the purpose of tracking and control. Neural networks are an effective algorithm for estimating non-linear system parameters, and recurrent neural networks in particular are quite useful for sequential, often time-based data. Long Short Term Memory (LSTM) neural networks [6] as types of recurrent neural networks are specifically designed for modelling time-varying systems with changing process characteristics.

This research effort is about investigating new ways in which machine learning techniques can be incorporated into algorithms in the area of real time cloud control systems, in order to improve robustness, quality and overall performance. 
One of these algorithms is the Kalman filter [5], which is an algorithm used to estimate unknown variables from known input measurements. One of the aims here was to characterize the effect of LSTMs on Kalman filter initialization and configuration, specifically when used to learn measurement and unknown variable noise correlation coefficients.

A motivation for this goal was to see if there were any performance gains, and to characterize the nature of these gains to provide clues for further enhancements. Overall, there were improvements in Kalman filter performance in various cases, such as for fast changing signals with exponential derivatives, and for tracking sinusiod signals, in which small gains in accuracy were realized.

Another aim was to determine how to efficiently model real time applications, and to characterize the benefits of a model-based cloud controller. Under what cloud environments are there gains or losses in performance? Using a model driven engineering approach, explore how to update these models during the application's life cycle, and use them to optimize performance in real time by implementing an adaptive controller. This controller will incorporate LSTM and Kalman Filter units for operating system and application tracking. The controller served as a case study of how these tools can be utilized in an industry standard cloud platform, to characterize the utility of these techniques in a real world environment.

To this end, Layered Queueing Networks (LQN) [16] was selected due to its popular use in the field of performance modelling. Queuing networks are systems of interconnected queues, and are layered when the service time at a node is determined by the response 
time of the subtending network at the node [99]. One of the advantages for having such a model in the controller is due to both the support for service time specification on inputs, and the ability to run scenarios. It also allows for bottleneck analysis in real-time. LQN driven decision making can account for deep complexity in the structure of the application, allowing for fine-grained computation of resource overcapacity and overutilization.

In the model-based controller's analysis, it was found that all virtual machine types had controller driven performance gains that were very sensitive to user specified thresholds. More nuanced threshold specification is recommended, tailored to each machine type. In the cloud system analysis it was seen that ad hoc user-specified trigger thresholds worked in around $90 \%$ of the cases in terms of enabling the controller to reduce the monitored application's resource footprint, and ensure adequate resource allocation. Overall, for proper performance of the controller, a targeted cloud system analysis is essential in determining optimal trigger thresholds and controller configuration parameters. A key contribution was to propose a scheme employing a well known algorithm called Principal Component Analysis (PCA) [100] in novel combination with the Kalman filter. PCA transforms a set of observation variables into a set of linearly independent variables (which form a basis). The aim was to potentially improve estimation accuracy by eliminating noise in the observed variables, and also benefit from ease of implementation. The combination of PCA and Kalman filters reduced the error rate in tests, where variance in absolute error (due to noise) is regularly reduced by an order of magnitude. This improvement in accuracy was influenced by the measurement noise levels in the test 
data used in the evaluation. The PCA-Kalman approach is more accurate because there are essentially two levels of noise reduction, when compared to a pure Kalman filter.

\subsection{The Problem and the Tools}

A system can be described as a set of objects which are interrelated, delineated by some kind of (spatial or temporal) boundary. Time varying systems are systems whose parameters change over time based on predefined laws or external factors like time-delayed or time-advanced inputs. Control of time varying systems such as running applications is a useful task for the purpose of resource management, maximizing utility and risk management.

Various control algorithms are used for this task, as outlined in the previous section. One of these mentioned is neural networks. Artificial neural networks are control algorithms based on biological neural networks. They are a graph structure of neurons with weights and biases, which are used to learn to execute tasks without any prior programming. They do this by observing large sets of examples as inputs during a configuration phase called training. Typically each neuron has multiple weighted incoming connections from other neurons, a non-linear activation function, and outgoing connections to other neurons. A recurrent neural network is one where input data is in similar repeating sub-structures that form a graph. The connections between the sub-structures form a directed graph. This conveys the ability to display temporally dynamic behaviour, where the internal state of the graph can determine behaviour, as much as inputs themselves can. The reverse edges of the graph provide feedback connections. 
Long-short-term-memory is a recurrent neural network that has specific repeating sub-structure involving a cell, input and output gates, and a forget gate. This allows the network to selectively purge internal state ('memory'), and to operate selectively based on internal state or inputs.

Another algorithm useful for control of time-varying systems is a tracking algorithm called the The Kalman filter (KF). Also known as linear quadratic estimation, it is an algorithm that estimates unknown variables from a series of measurements. These measurements typically contain noise and other inaccuracies. The extended Kalman filter is the nonlinear version of the $\mathrm{KF}$ that estimates using estimates of the mean and variance of measurement values.

\subsection{Research Questions and Goals}

One of the main issues with tracking applications which are time varying systems is changing process characteristics, that create a need for the controller to recognize these changes and retune itself [93]. Machine learning algorithms are an effective class of algorithms have been applied to this problem. Recurrent neural networks are an effective algorithm for estimating non-linear system parameters on sequential data from real time systems. Long Short Term Memory neural networks [6] as types of recurrent neural networks are specifically designed for modelling time-varying systems.

These research goals are about how machine learning techniques can be incorporated into Kalman filters on real time control systems to improve performance in the cloud. These goals include: 
1. Characterize and quantify the effect of LSTM neural networks tuning on Kalman Filter performance, specifically when used to learn measurement and process noise coefficient matrices. Are there any performance gains? (Section $8.2 \& 8.4$ )

a. Explore various LSTM learning methodologies (linear regression versus logistic regression - classification) and their effects on noise coefficient matrix discovery (Section 8.2)

2. Determine how to efficiently model real time applications (Section 6.1.5.1). Explore how to update these models during the application's life cycle. Use the updated models to optimize performance in real time, essentially using the model (Section 6.1.4.2):

a. To predict application state

b. To reason about and select optimal actions

Model parameter estimation from measurement data is necessary for model tracking. The proposed algorithm for model parameter estimation is principal component analysis (PCA - chapter 4). There is risk associated with PCA around the scores (i.e. the corresponding eigenvalues) of the principal components. Small eigenvalues are indicative of a lack of information associated to the corresponding dimension and eigenvector. Overcoming this risk is the assumption that there are more measurements than model parameters, and that the measurements contain sufficient information about the unknown model parameters.

3. Implement an adaptive controller using the LSTM and Kalman Filter (Section 6.1). 
a. Evaluate performance gains, exploring two main modes of adaptive control (Section 6.1.4)

i. Active control where control actions are taken whenever thresholds are crossed (Section 6.1.4.1)

ii. Passive control where application monitoring occurs, measurements are collected but no control actions are issued (Section 6.1.4.1)

b. Create a specification format for application control commands (Section 6.1.5). This should enable a separation of concerns between the control logic and design, and application specific scripts and commands. It will also allow for a ubiquitous system portable to any operating system and cloud environment. System administrators will use this format to configure the controller for their particular environment.

\subsection{Contributions}

These are the contributions made in the course of this thesis:

1. Characterized the effect of LSTM neural network tuning on Kalman Filter measurement and process noise coefficient matrices (KF-LSTM - Section 8.2 \& 8.4). 
a. Explored various LSTM learning methodologies for KF noise coefficient matrices, linear regression (KF-LSTM1) and logistic regression (KF-LSTM2) in section 8.2.

2. Proposed a control algorithm (Section 6.1.4.2) using LQN model-driven actions to monitor and control a real application:

a. To predict application metrics using KF-LSTM1 algorithm (Section 8.4)

b. To reason about and select optimal actions (Section 6.1.4.2)

3. Implemented an adaptive controller using the LSTM and KF (Section 6.1), and evaluated performance gains, exploring active and passive modes of adaptive monitoring (Section 8.7)

a. Create a specification format for application control commands. (Section 6.1.5).

4. Explored how to track these models during the application's life cycle. Proposed PCA for model parameter estimation from measurement data (Chapter 4).

\subsection{Thesis Organization}

The thesis is organized into the following parts:

1. Introduction, background and a review of related research

2. PCA Layered Queueing Network (LQN) parameter inference algorithm

3. The controller and its components 
a. LSTM Unit for learning short and long term prediction horizons system measurement data, and tuning Kalman filter input parameters

b. EKF Unit for tracking system measurements, inferring of application state, and making predictions about future system state

c. Control Unit for computation of control actions and orchestrating application and resource instances

d. Configuration \& Application Unit for interacting with the operating system, monitored application and its resources

4. Experiment plan

5. The controller testbed and its components

6. The model tracking testbed and its components

7. Components analysis

8. Controller evaluation

9. Conclusion 


\section{Chapter 2: Background}

\subsection{Machine Learning For Kalman Filters}

Kalman filters are a popular tool for state estimation of systems where measurements are generally noisy. However they require a measurement and model to be specified which requires modelling work, and the models themselves are approximations of the real world [17]. In order to estimate and track dynamic systems, temporal filters like Kalman filters are especially good at this. Ignoring temporal information can lead to very noisy estimates and potentially lead to feature confusion.

Measurement and transition models need to be specified apriori in these types of problems, and to this end Kalman Filters are poor, hence the need for model estimation techniques, like the use of long short term memory (LSTM) [18] and support vector machines (SVM) [19]. The architecture proposed here tackles the problem of learning the internal model of the system being tracked in order to lead to enhanced Kalman filter performance.

Some techniques for noise covariance and model estimation include static optimization. For example for pose estimation, a Levenberge-Marguardt optimizer was used by Du et al [21] to find dictionary coefficients that minimize the 2D backprojection error on the RGB input frame. That allowed temporal information in joint velocities be used to sanitize joint position estimates.

Dynamic regression of Kalman filter parameters has been studied, such as using support vector regression to estimate parameters at each filter prediction step [20]. In the research 
effort by Salti and Di Stefano, much like in this project, the noise covariance and state transition matrix models are learned jointly and updated during state updates. Their system is able to handle time-varying data and outperforms manually tuned Kalman filters in their benchmarking tests.

Bayes estimators like Kalman filters have been used in sensor networks to integrate multiple sources of information and improve estimates of unknown factors [22]. 'Deep' Kalman filters (second order Extended Kalman Filter) have been used that project to integrate information from Global Navigation Satellite System (GNSS) and Inertial Measurement Unit (IMU) sensors, to ultimately outperform basic Kalman filters. Estimation of unknown desired signals from a run of data from a known signal is a problem also known as online regression [23]. Neural network-based regression algorithms are able to compute non-linear and complex models [24], especially so with deep neural networks [25]. These are unable to capture time dependencies in processing temporal data and time series [26], hence recurrent neural networks are introduced in order to handle memory from past information [27].

Second order gradient descent schemes provide superior performance to first order approaches like backpropagation recursion [28]. A good example of a second order approach is the decoupled Extended Kalman Filter (EKF), which has been shown to combine with recurrent neural networks (LSTMs) to perform well in problems not easily solvable by traditional RNNs [29]. This combination of and EKF is done where EKF is used as a means of updating feedback weights, and is thus an alternative to gradient descent. Whereas this thesis also uses LSTM and EKF, it uses gradient descent algorithm 
rather than decoupled EKF training used in this approach. These higher order approaches have higher complexity and suffer from saddle points in the neural network gradient descent [30]. There is a tradeoff between neural network complexity for higher order techniques (like EKF), and saddle points in lower order approaches [31]. Exploding and vanishing gradient problems can occur due to long term events [32], where second order training methods provide stronger performance at the cost of higher complexity.

Vanishing gradients are caused by long time lags between events, and refer to errors moving back in time which exponentially decay, while exploding gradients refer to those which exponentially blow up. Particle filtering techniques are one way of reducing the complexity of second order techniques to the order of first order techniques for training RNNs, while maintaining the same performance [23].

Particle filtering of this kind, for example Rao-Blackwellized filtering, can be used in such problems of tracking series of noisy measurements from multiple sources like sensors [34]. The problems are simplified via sampling (i.e. Monte Carlo sampling), and reduced to single sample sub-problems solvable techniques like Kalman filtering, which are outperformed by LSTMs for single source tracking by 2x [33]. Probabilistic LSTM tracking of multiple targets is shown to be significantly better than vanilla Kalman filter tracking. Some of the top performing alternatives on the KITTI benchmark include a tracking-by-detection method where computer vision techniques are used to curate detections between datasets (frames), whereas local interest point detectors are used along with optimal flow algorithms to create point trajectories within datasets [35]. Computing the min-cost flow trajectory between nodes in the detection graph is another 
improvement of this technique [36], where connections between nodes are inferred through a quadratic interactions, and the graph model parameters are learned using structured support vector machines (SVM). Updating of model parameters has been done using Markov decision processes with reinforcement learning for state transitions. If the multiple target tracking problem is considered as a two step process, individual object trajectory tracking, and multiple trajectories puring steps, Blackwellized particle filtering works best with LSTM, which performs twice as well as K-nearest neighbour and Kalman filtering on the individual object tracking step. However for multiple object tracking, LSTM and Kalman filtering performed equally well for end-to-end tests on the KITTI benchmark [33].

\subsection{Long Short Term Memory Cell [109]}

Nonlinear state space models are a recurrent pattern in real time systems, especially where sequential event streams are to be modelled. Transition and generative distribution patterns are to be taken into account for effective predictive modelling. Hidden Markov Models, and Recurrent Neural Networks are popular techniques for modelling such distributions, usually Gaussian in nature [10]. In this effort, LSTM neural networks were considered as holding potential in modelling time-based event streams such as are apparent in real time cloud compute systems.

The structure of each LSTM cell [109] is illustrated below: 


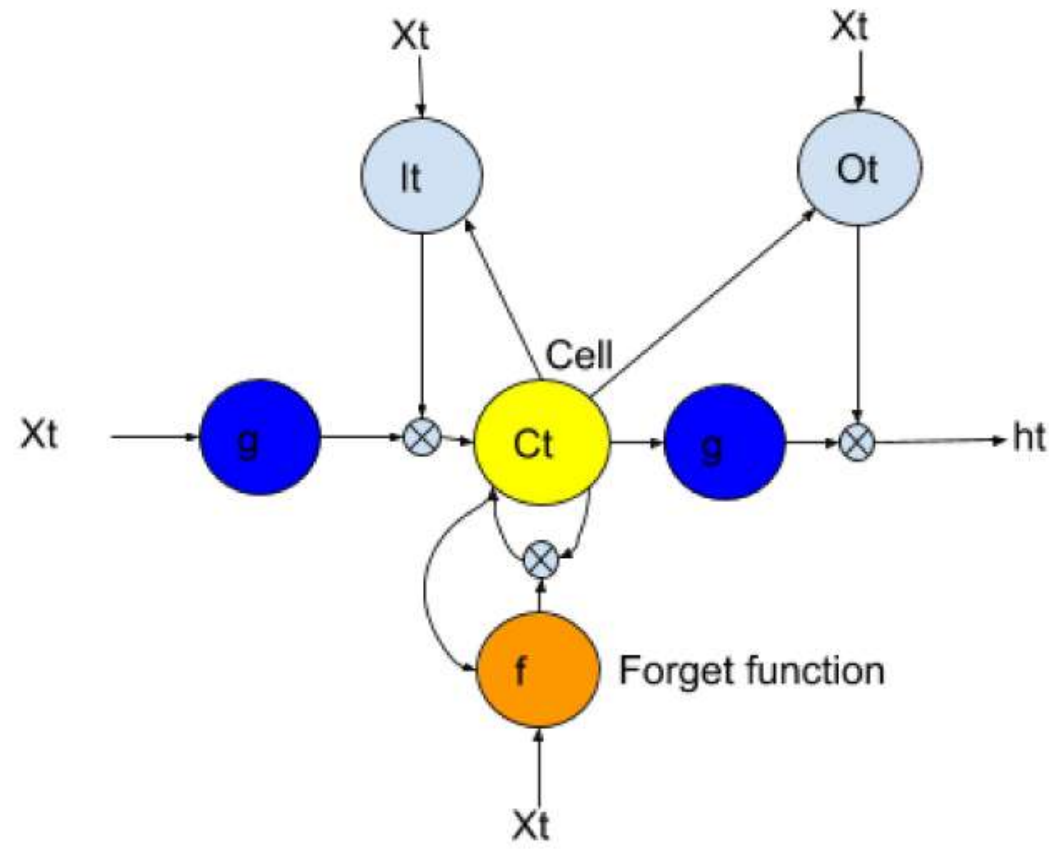

Illustration 2.2 LSTM Cell Structure[109]

Each individual cell consists of the input signal (encoded input measurements as provided by controller unit) encoded to a 0 to 1 scale. The signal is then passed through a nonlinear function (g) which performs an integral (a key component of a neural network cell). This nonlinear output is then convolved with the original signal through a multiplier and into the memory cell $(\mathrm{Ct})$.

The memory cell contains a feedback input that encodes long term memory, as well as input from a forget function which performs the role of purging select data in the memory unit (i.e. forgetting). This is key to ensure the LSTM cell can discard memory information which is efficient for increasing accuracy. 


\subsection{Extended Kalman Filter}

The Extended Kalman Filter (EKF) consists of an estimation and an update block. From the system overview, it orchestrates the tune and update operations, taking the measurement $(\mathrm{R})$ and process noise coefficient $(\mathrm{Q})$, and state transition $(\mathrm{F})$ matrices as tuned input. It also takes in measurements (z) as update inputs, and produces predictions $\left(\mathrm{x}_{\mathrm{k}-1}\right)$ used by the application monitor for decision making.

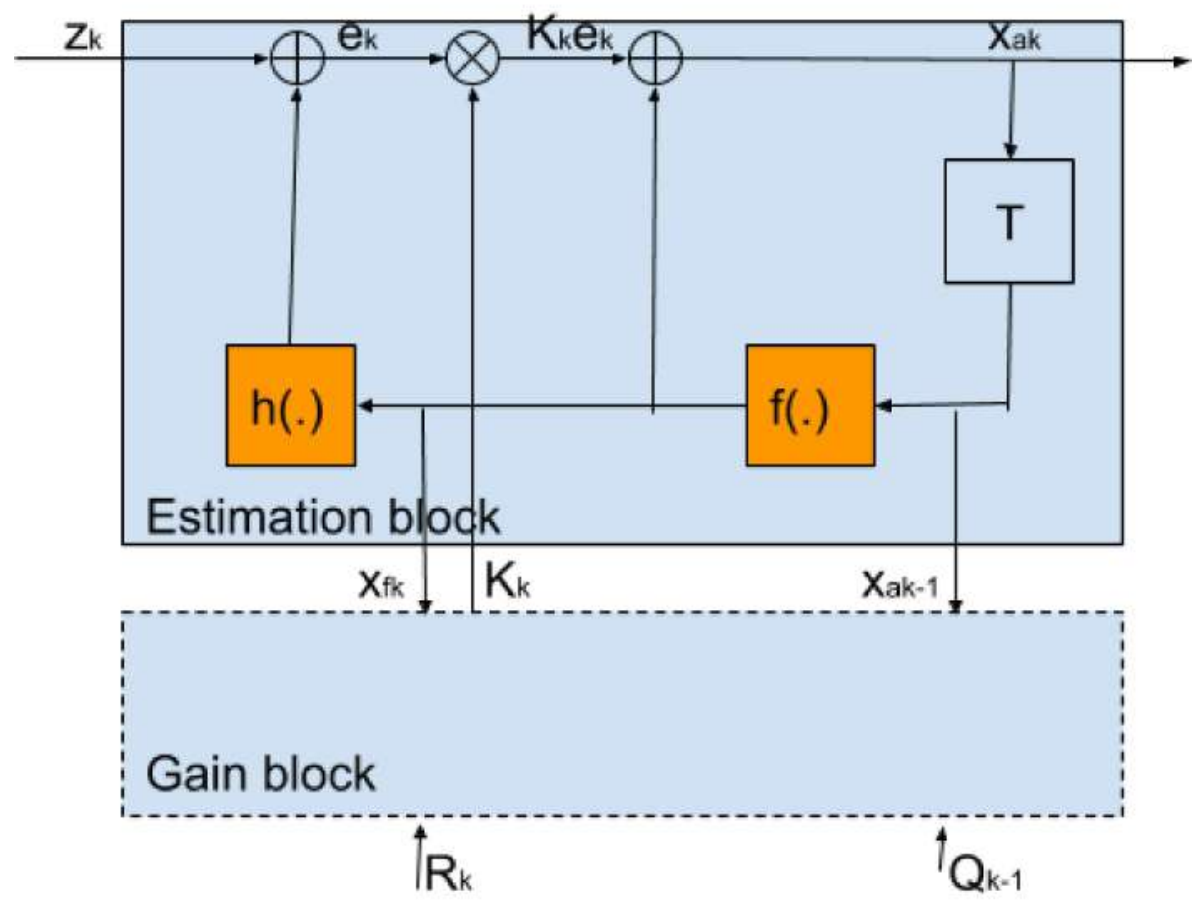

Figure 2.3: Extended Kalman Filter diagram [5]

Its operation includes predictor and update steps that attempt to predict the state of a nonlinear system described by a difference equation and observation model with noise.

$x_{k}=f\left(x_{k-1}\right)+w_{k-1}$

$z_{k}=h\left(x_{k-1}\right)+v_{k}$ 
$\mathrm{x}_{\mathrm{k}}$ is the state vector at time step $\mathrm{k}, \mathrm{w}_{\mathrm{k}}$ is the process noise vector, $\mathrm{z}_{\mathrm{k}}$ is the observation vector and $v_{k}$ is the measurement noise vector. With $E\left[w_{k} w_{k}^{T}\right]=Q_{k}$, and $E\left[v_{k} v_{k}{ }^{T}\right]=R_{k}$, $f($.$) is the process nonlinear vector function, h($.$) the measurement nonlinear vector$ function and $\mathrm{J}^{\mathrm{f}}$ is the Jacobian of $\mathrm{f}($.$) .$

The state forecast error of the EKF is given as

$$
\begin{aligned}
e_{k} & =x_{k}-x_{k}^{f} \\
& =f\left(x_{k-1}\right)+w_{k-1}+f\left(x_{k-1}^{a}\right) \\
& \approx J^{f}\left(x_{k-1}^{a}\right) e_{k-1}+w_{k-1}
\end{aligned}
$$

The optimal forecast error covariance is

$$
\begin{aligned}
P_{k}^{f} & =\left[e_{k}^{f}\left(e_{k}^{f}\right)^{T}\right] \\
& \approx J^{f}\left(x_{k-1}^{a}\right) P_{k-1} J^{f T}\left(x_{k-1}^{a}\right)+Q_{k-1}
\end{aligned}
$$

Where $x_{k}^{a}$ is the optimal state estimate and $x_{k}^{f}$ the state forecast,

The goal of this to minimize the state estimate error $\mathrm{e}_{\mathrm{k}}$. The update step, posterior error estimate and covariance are other key steps of the EKF algorithm [4].

\subsubsection{Measurement Matrix \& Its Jacobian}

The Measurement function $h\left(x_{k}\right)$ is used to calculate a measurement from a state (eq (2)).

$z_{k}=h\left(x_{k-1}\right)+v_{k}$ 
This function can be nonlinear. The Jacobian $\mathbf{J}^{\mathrm{H}}$ is used to estimate the desired value of $\mathrm{H}_{\mathrm{k}}$ around values of $\mathrm{x}$ at time step $\mathrm{k}$, specified as a matrix of partial derivatives. $\mathrm{H}_{\mathrm{k}}$ is then used in place of $h\left(x_{k}\right)$ in the above equation.

The Kalman Filter itself uses the identity function as $\mathrm{f}($.$) using the actual operating$ system metric (from the 'top' command) as measurements (z) and filter states (x). The state prediction then represents the next expected operating system metric. Determination of the inferred application state variable is then done using principal component analysis and least squares estimation.

\subsubsection{Kalman Filter Error}

The prediction error for the EKF is given as :

$$
\frac{\left.\mid \widehat{z}(i)_{k}-\widehat{h(x}(i)_{k \mid k-1}\right) \mid}{N(i)}
$$

where $\widehat{x}(i)_{k \mid k-1}$ is the prior state prediction for process metric $\mathrm{i}$, and $\widehat{z}(i)_{k}$ is the measurement at step $\mathrm{k}$, and $\mathrm{h}\left(\mathrm{x}_{\mathrm{k}}\right)$ is the measurement function.

\subsection{Kalman Filters For Model Parameter Tracking}

Controllers can make intelligent changes to systems based on descriptions of the systems known as system models [102]. These include dynamic regression models by Hellerstein 
and co. [103] used in text editors and thread managers, and queueing network models [104] for predictive models. Combinatorial approaches like hierarchical autonomic architectures [105] have proved useful in capturing system changes using dynamic models and making predictions using layered queueing networks. That approach uses the EKF to track changes in the software system in order to keep the predictive performance model up-to-date. This applies best to transactional systems which are described as closed models. Generally with increasing numbers of samples, there are reductions in error and convergence in estimated model parameters (demand times, numbers of users) both in actual values and in variance. Varying the noise coefficient matrices on the measurement and parameter variables ( $\mathrm{P} \& \mathrm{Q}$ respectively) by over four orders of magnitude did not affect the behaviour of the estimator. One key advantage of this approach is the ability to use incomplete or sparse measurement data, and to accommodate different types of measurements. It was also robust to the use of a model which merely approximates the real software system (i.e. missing one or two components), and was robust to bottlenecks in the system, which usually cause loss of sensitivity to workloads and therefore cause model-divergent system behaviour. Self-managing computer systems [106] are another approach to model driven control not dissimilar to the hierarchical autonomous architecture. This includes a service demand computation, a workload analyzer, quality of service (QoS) control algorithm and a performance model solver. Computed service demands are used as inputs to the queuing model solver, while the workload analyzer computes the request arrival rates for the current and next time steps using statistical analysis (Markov Chain and queuing model) 
on the running application. The controller then solves the performance model to get a QoS value for a set of points around the inputs (arrival rates and demand times) to get the optimal value, which is then used to create reconfiguration commands sent to the application by the controller. Overall the QoS value for the controlled application improved to remain stable even under high workloads for all the search (hill \& beam) algorithms.

These approaches are similar to this research project in the overall components. There is a computation of service demands and workloads, a search of the optimal related performance model and controller decisions about optimal reconfiguration actions. The key differences are in the details of each component, especially in the computation of service demands and workload (EKF, Markov Chain), and in the optimization of the QoS value (layered queue networks, hill/beam search). When compared to this thesis, the hierarchical autonomous controller is closer in its components, with the key difference being the combination of EKF with principal component analysis for determining service demands and model parameters, and the use of machine learning techniques in configuring the measurement and process noise coefficient matrices, state transition and measurement matrices. There is also added analyses of multiple machine learning schemes, and a cloud based virtual machine-type controller analysis. 


\section{Chapter 3: Review Of Related Work}

\subsection{Machine Learning \& Kalman Filters}

Kalman filters have a smoothing feature that makes them more desirable than other non-linear predictive techniques like neural networks when discontinuities in outputs are highly undesirable. This is why they are used for time series prediction in this thesis. The sub-problem to tackle is how to estimate the noise coefficients for the measurement and process functions of the Kalman filters. The techniques surveyed below shed some light on other combinations of Kalman filters with machine learning in order to highlight the strengths and deficiencies of these algorithms. They show how LSTM in particular is used for time series prediction, multiple regression, and shed light on some of the issues involving training cost, loss functions and learning rates of LSTM.

\subsubsection{Model Extraction From Sequential Data}

The unscented Kalman filter has been used with LSTM networks for tracking nested interval noisy hand gesture data with reasonable success [37]. Noise in the sequential data is revised by using two sampling intervals to alter the sigma distribution and improve amelioration accuracy. Key components of this approach include an efficient model [39] for noise detection, nested interval unscented Kalman filter for noise reduction (NIUKF), and the NIUKF-LSTM network for detection of hand gestures from noisy data. The unscented Kalman filter is a combination of a nondestructive transformation and a 
Kalman filter, where the transformation is a way of calculating statistics (sigmas) of a random variable (KF state transition) that undergoes a nonlinear transformation. Convolutional, bidirectional, long short term memory networks (CBLSTM) have been shown to enable learning of machine health monitoring methods from raw machine data [40]. A type of deep neural network, these employ convolutional neural networks (CNNs) to extract robust local features from raw sensory input, and LSTMs to capture longer term effects and model time dependencies. The bidirectional structure allows for past and future relationship inferrance. Where model extraction is very difficult, methods like Markov models and Kalman filters are seen to be ideal for handling sequential data, especially those with raw time series [41-42]. CNNs are able to encode more critical information localized in sequential data, as well as compress the length of the input sequence, enabling subsequent RNNs to capture higher quality time-based information. LSTM encoder-decoder systems (LSTM-ED) have been used to extract models (health index - HI) and reconstruct these models back to time series data [55]. This is in the domain of creating a health index for in an unsupervised fashion for the purpose of estimating the Remaining Useful Life (RUL) of health equipment using operational multi-sensor data. The reconstruction error on the $\mathrm{HI}$ is then used to determine the RUL due to prior knowledge of the expected error accumulation over time. Other schemes have been used to determine the remaining useful life using sensor data via estimation models [56], using wavelet neural networks \& computational estimation techniques. A variant of LSTM-ED has been used for sequence to sequence learning tasks like in learning phrase representations [58] and general sequence-to-sequence translation [59]. The system 
consists of an LSTM encoder which maps a sequence of sensor data to a fixed length vector representation, and a LSTM decoder which maps this vector to the target representation. This has been used in anomaly and novel subsequence detection. Linear regression is then performed on the $\mathrm{HI}$ curves, and ordinary least squares method is used to determine the distance between the extracted curves representing the current system state, and the curves of a perfectly healthy system. These distance parameters are the indicator of system health. C-MAPSS Turbofan Engine Dataset [60] and the Milling Machine Dataset [61] were used to do evaluation. There were fairly accurate reconstructions in the C-MAPSS dataset with significant variations in reconstruction error per dataset machine $(23 \%, 17 \%$ and $23 \%$ on some LSTM-ED models), and the prediction error increases with an increase in RUL. It was concluded that significant correlation between reconstruction error and cost incurred indicates that reconstruction error is able to the health of the mill [55].

\subsubsection{LSTM For Anomaly Detection \& Kalman Filters For System State Tracking}

A Metromechanical Inertial Measurement Unit (MEMS-IMU) is usually paired with GPS in order to mitigate nonlinear errors in measurements [44-45]. However GPS signals are not always available or are blocked, so errors persist. As alternatives, learning approaches were tested. LSTM-RNN denoising outperformed Auto Regressive and Moving Average (ARMA) with fixed parameters, reducing standard deviation on gyroscope readings by $42.4 \%, 21.4 \%$ and $21.4 \%$, and reduced attitude errors by $47.6 \%, 42.3 \%$ and $52.0 \%$ [43]. 
Anomaly detection on real time light curve data from satellites has been done using LSTMs in the Ground-based Wide Angle Camera array telescope [46]. In this problem, LSTM results are more reliable if the light curve time series is stationary, hence the need to process differences between time steps rather than raw luminescence data. Kalman filtering is seen as an optimal approach to tracking system states from light curve observation data including noise and interference [47].

\subsubsection{LSTM \& RNN Training Cost, Loss Functions \& Learning Rates}

LSTM neural networks have been used for statistical parametric speech synthesis (SPSS) for mobile environments with optimizations for reduced computation and disk footprints [48]. There was increased robustness to errors in training data. However the LSTM-SPSS was significantly slower than Hidden Markov Model (HMM) based SPSS in terms of real time ratio [49]. Some of the optimizations included weight quantization to 8 bit integers (from 32 bit floating point numbers), multiframe bundling for inference to reduce computation costs, and robust linear regression. Learning with linear regression using squared loss functions can suffer from outliers like long tails [50], which in the realm of speech processing can come from recordings, transcriptions, and extraction errors. Linear regression with heavy-tails, or minimum density power estimator [52], do reduce the effect of outliers. $\epsilon$-contaminated Gaussian distributions can be used to reduce outlier effects on Gaussian error distributions [53]. In the results, disk footprint of the LSTM-RNN was reduced from 1.05 MB to $272 \mathrm{~KB}$. Multiframe inference required a reduction of learning rate (1e-5 to 2.5e-6) with a bundling of 4 frames, leading to a $40 \%$ 
reduction in walltime. The $\epsilon$-contaminated loss functions allowed for an increase in learning rate (2.5e-6 to $5 \mathrm{e}-6)$ with a similar or better naturalness when compared to the squared loss function. Overall the LSTM-RNN was $15-22 \%$ slower than the HMM-RNN system in terms of total synthesis time (on older devices), but was able to produce more natural sounding speech [54].

\subsubsection{Improving Time Series Prediction Using LSTM}

Predicting short term travel time uses real travel time values in a sliding window to predict travel times several steps into the future. Non-stationary effects and abrupt changes in travel time can make predictions less accurate. Long short term memory with deep neural networks (LSTM-DNN) has been applied to this problem [62]. This method was applied to the Caltrans Performance Measurement System (PeMS) 90 day travel time dataset, and compared to linear models like linear regression, Ridge and Lasso regression, autoregressive integrated moving average (ARIMA) [63] and DNN. Two categories of methods had been applied to the problem: statistical methods like ARIMA for traffic flow parameter prediction [64], and machine learning methods. Variants like H-ARIMA+ [65] and spatio-temporal (ST-ARIMA) have also been explored. Bayesian networks for traffic flow prediction [68]. Machine learning techniques like local online kernel models for Ridge regression, support vector regression, DNNs [70] and LSTMs with gated recurrent units (GRUs) have been applied to traffic flow prediction problems. Deep belief networks (DBN) have been proposed together with DNN models for traffic flow prediction [71]. A common issue in these studies is the need to study the impact of varying hyperparameters 
of the models on accuracy and overall performance. In this research effort [62], short term travel time predictions of 16 LSTM-DNN models were built by applying 16 combinations of 3 hyperparameters (number of LSTM cells, layers and DNN fully connected layers) over 128 experiments. It is found that increasing depth and width does not lead to gains, while maintaining a middle scale deep learning layer leads to more accurate and consistent prediction results in mean absolute percentage error (MAPE) and root mean square error (RMSE). When compared against regression techniques like linear regression, Ridge and Lasso regression, LSTM-DNNs show superior performance producing useful predictions even when sliding windows are narrow and prediction horizons are far ahead, with both MAPE and RMSE lower than benchmark methods at the time of the study.

\subsubsection{Multiple Regression \& Feature Selection}

A happiness intensity prediction challenge submission was made to the Emotion Recognition in the Wild challenge [72], employing multiple regression. Images of groups of people in a social setting were to be analyzed to determine happiness level. Convolutional neural networks were used to extract facial features, and RNNs were used to memorize important features and predictions. A RMSE reduction of 0.5 units on the baseline algorithm (which used only global information) was observed in benchmarking. The Happy People Images dataset (HAPPEI) [73] was used, which provides group and individual level feature labelling like group happiness \& occlusion intensity, and location. 
Existing work on group level analysis involved using the Riesz transform and local binary pattern as features, with continuous random fields as group models [74]. Other researchers performed group level arousal and valence recognition by using face, body and context information [75]. This challenge came with the CENTRIST [76] baseline approach which has scene descriptors per image. These should capture background, foreground and face descriptors. This study proposes an improvement model on CENTRIST where face descriptors are given priority over scene descriptors, as they are considered more important in happiness level determination. The proposed method selectively memorizes features considered important for happiness prediction, and forgets the rest. Off the shelf CENTRIST is used as the scene descriptor, an 18 layer residual CNN (ResNet-18) as the face features, trained on face emotion recognition, and LSTM learns faces continuously from sequences of these scene and face descriptors. For happiness level prediction two kinds of regression are done using LSTM neural networks: linear regression and ordinal regression. Linear regression uses mean square error as the loss function, while ordinal regression uses a combination of 6 binary classifiers where each output is a truth indicator of whether the happiness level is greater than the classifier's rank. To improve facial recognition performance, a facial landmark detector [76] was employed. Locations of eye \& mouth centres are used in an affine transformation, occlusion issues in eyes are tackled using the filtering mechanism of the facial landmark detector. An alignment pipeline is used to crop and align the filtered faces, which reduces the LSTM RMSE from 0.546 to 0.516 . Feature aggregation was the next optimization step, done using mean encoding, where the average of the face features 
as the representation of the image. Adding scene features to the model reduced the RMSE further from 0.515 to 0.509 . Ordinal regression does better than linear regression overall (RMSE of 0.489 versus 0.494 ), and a combined approach is the best, showing the two are complementary.

\subsection{Principal Component Analysis}

Input variables to learning algorithms can be partially linearly dependent. This collinearity inhibits regression analysis, and it gets worse as the degree of correlation increases. One way to reduce this dependency is to project the variables onto a lower dimensional subspace. Principal Component Analysis (PCA) is one way to do this, by using the matrix of correlation coefficients, decomposed into eigenvectors which are sorted by eigenvalue score. The most significant of these can then be selected as the lower dimensional subspace, a form of model order reduction.

Cross-Gramian based frequency-weighted model order reduction [90] was an augmentation of the balanced truncation algorithm [88] to enable error minimization over desired frequency ranges. It is more stable and requires less computation than other techniques. Independent Component Analysis (ICA) [91] is a popular dimensionality reduction technique that is usually used together with two dimensional principal component analysis (PCA) [91]. Two dimensional PCA is usually used here in preprocessing to reduce computational complexity for ICA. In the image processing facial recognition task by X. Zhang \& X. Ren, the combined approach performed better than classical PCA, 2dPCA and ICA algorithms in isolation for processing both images 
and audio in raw format and vector form. The combination of PCA and fast ICA has been used with K-Nearest neighbour classification in the task of face recognition [111]. The PCA and fast ICA algorithms are used for dimensionality reduction and mixed matrix projection to the feature space, and fed into the classifier for good results on the ORL face database [94].

Principal Components Analysis (PCA) has been used for multiscaled local image orientation estimation in conjunction with Kalman filters [95]. PCA is used to find the maximum likelihood (ML) local image orientation, and Kalman filter is used for multiscale pyramid decomposition. The combined approach proved very robust against noise compared to other state-of-the-art techniques, and is applicable in computer vision, image segmentation, texture analysis, optical flow analysis and other areas of image processing. PCA has also been used in combination with Kalman filters for on-line aircraft engine diagnostics [96]. Three algorithms are compared here, Kalman filter, PCA and combined approach. The Kalman filter approach directly estimates faults from turbine readings, while the PCA is used to determine the identity of the faulty component (categorical classification). The combined approach uses the PCA tool to determine the process noise coefficient matrix of the Kalman filter via weighting, a modification of the Robbins-Monro scheme for estimating Kalman innovations [97]. The key benefit of the combined approach is to mitigate the combined errors of the individual approaches, where the weight vector prevents destabilization of the filter in case of bad fault detections in the PCA. 
Principal Component Analysis (PCA) is one of the classic dimensionality reduction algorithms [92], providing minimal mean square error and a linearly independent basis in the target subspace. PCA has been used in linear system analysis for model reduction [88]. It has been developed as a way of analyzing signals through singular value decomposition. Kalman minimal realization theory [89] is recast using this approach to analyze the sensitivity of subspaces to small perturbations in associated models. Typically for perturbed models there is no lower order model with the same impulse response. The main advantages of principal components analysis (and single value decomposition) is it provides convenient tools for coping with multiple signals (also, limitations in physical hardware can easily be explained in terms of signals).

\subsection{Autoscaling Of Microservices}

Below is a representative research effort in the area of autoscaling of microservices based on their unique qualities. Microservices are a cloud-based software architecture leveraging decoupling of services and decentralization of resources [78] popular in software lifecycle management.

Workload forecasting and optimal resource allocation are key obstacles in autoscaling. A model-predictive algorithm for workload forecasting, involving look-ahead optimization, is a useful approach to tackling these issues [112]. Heuristics-based virtual machine allocation and migration [113] and has been used to provide dynamic provisioning for multi-tier applications using queueing network models. These approaches focus on 
minimizing cloud resource footprint and also tier-based cost with the introduction of a pricing model. Other approaches include the use of control-theoretic algorithms where a tiered application is black-box profiled by tier to build approximate models which characterize resource needs that are then used to automatically provision virtual machine resources, coined as autonomic management of virtual machines [114]. Also included are load balancing controllers, and elastic management of cluster-based services [115], which autoscales resources with minimal impact on the user. Workload based and profile based approaches have also been explored to solve the problem of just-in-time scalability. These approaches attempt to increase throughput of the application only at the expense of the cloud provider, or they do not provide multiple classes of service, where multiple classes of service are on a single virtual machine. These are potential limitations on this research effort too, but the key distinction is that in this project these concerns are decoupled from the main controller. The user has complete responsibility for choosing the application model, threshold type and criteria, and scaling application tiers and target virtual machine distribution. The user might not be able to account for the overall utility value to the cloud provider though, so this is a shared limitation.

Independently monitoring and scaling a subset of microservices using resource demands from packet sniffers and layered queueing models for dynamic computational optimization is the approach taken in ATOM [77], a controller employing model driven scaling of microservices. Other approaches to autoscaling include the use of meta-heuristics [79], application profiling and analytical methods [80], but these do not take platform-specific effects into consideration. ATOM uses layered queueing networks 
(LQN) [81] with Bard-Schweitzer single step mean value analysis to reason about potential workload changes and their impact on subsystem states, and make appropriate scaling decisions (a similar approach used in this thesis). Searching for optimal workload selections to minimize resource usage is done via genetic algorithm (whereas this thesis uses gradient descent). Compared to simple rule based controllers, ATOM has $26 \%$ to $45 \%$ higher throughput, using vertical and horizontal scaling actions only. 


\section{Chapter 4: PCA Model Tracking Algorithm (KF-PCA)}

Estimation of model parameters is a problem relevant to the task of modelling and tracking real time systems, one of the key research goals above. The Kalman filter model tracking with PCA (KF-PCA) algorithm is introduced which provides a means of estimating model parameters from Kalman filter predictions. The aim is to potentially improve estimation accuracy by eliminating noise in observed variables, and also benefit from ease of implementation. The goal of KF-PCA here is to estimate the LQN model parameters given the measurement data.

The combination of PCA and Kalman filters could reduce error rates when compared to a pure Kalman filter because there are multiple steps of noise reduction, such as PCA's eigenvector decomposition and dimensionality reduction, and also the Kalman filter measurement and state process noise reduction steps.

\subsection{PCA-LQN Parameter Inference From Kalman Filter Predictions}

This section covers the interpretation of the output of the Kalman filter. Projection from operating system measurement predictions to model (LQN) parameters is used to estimate the model parameters when model inputs are not completely known. Measurements of the operating system state (using the 'top' command) are used by the host monitor to make inferences about the internal state of the application under observation. This is done using the KF state prediction prior to update $\left(\widehat{x}_{k-1}\right)$. 
This prediction and the measurement set it belongs to are then passed through principal component analysis (PCA) to determine the covariance eigenvectors where the number of components is the number of (LQN) model parameters to be inferred.

A least squares linear system solver is then used to project the principal components to the model state space to determine the LQN model parameters associated to the Kalman Filter prediction $\left(\widehat{x}_{k-1}\right)$.

A summary of the algorithm is shown in the figure below. The derived state $p_{k}$ is used in the controller for threshold crossing determination. The PCA factor, $\mathrm{m}_{\mathrm{LS}}$, is stored in the application state file after tuning, and used later in operational mode. When new measurements are taken, a PCA is calculated and used with the saved $\mathrm{m}_{\mathrm{LS}}$ vector as described above until the next tuning phase.

Given:

$\mathrm{n}_{\text {comp }}=$ number of principal components, $\mathrm{p}_{\mathrm{k}}=\mathrm{LQN}_{\mathrm{p}}=$ layered queueing network model solver parameter vector, $\mathrm{z}_{\mathrm{k}}=$ operating system metrics vector from 'top' command ( $1 \times 24$ vector) $\mathrm{x}_{\mathrm{k}}=$ Kalman filter's current state matrix $(10 \times 24$ matrix - including 9 previous states $)$ $\mathrm{x}_{\mathrm{k}-1}=$ Kalman filter state prediction prior to update, where it is tracking $\mathrm{z}_{\mathrm{k}}$, and has the identity state transition function $\mathrm{f}().(10 \times 24$ matrix - including 9 previous states $)$ $\widehat{x}_{k-1}=$ normalized $\mathrm{x}_{\mathrm{k}-1}$ $\boldsymbol{\sigma}^{\mathrm{xk}}=$ Covariance matrix for $\mathrm{x}_{\mathrm{k}}$, each entry is a covariance between a row and column $\mathrm{PCA}^{\sigma \mathrm{x}}=$ Principal components matrix for covariance matrix $\boldsymbol{\sigma}_{\mathrm{xk}}$ 
$\mathrm{m}=\mathrm{PCA}^{\sigma \mathrm{x}}$ to $\mathrm{LQN}^{\mathrm{p}}$ factor, a series of time-based weights applied on a variable's history $\mathrm{m}_{\mathrm{LS}}=$ Least squares linear system solution of $\mathrm{m}$,

$$
\begin{gathered}
\text { Define } n_{\text {comp }}=\left|L Q N^{p}\right| \\
x_{k}=\left[\begin{array}{ccccc}
x_{11} & x_{12} & x_{13} & \ldots & x_{1 n} \\
x_{21} & x_{22} & x_{23} & \ldots & x_{2 n} \\
\ldots & & & & \\
x_{d 1} & x_{d 2} & x_{d 3} & \ldots & x_{d n}
\end{array}\right] \\
=>\sigma^{x_{k}}=\left[\begin{array}{ccccc}
r_{11} & r_{12} & r_{13} & \ldots & r_{1 n} \\
r_{21} & r_{22} & r_{23} & \ldots & r_{2 n} \\
\vdots & \vdots & \vdots & \ddots & \vdots \\
r_{d 1} & r_{d 2} & r_{d 3} & \ldots & r_{d n}
\end{array}\right] \\
=>P C A^{\sigma^{x}}=\left[\begin{array}{ccccc}
p_{11} & p_{12} & p_{13} & \ldots & p_{1, n_{\text {comp }}} \\
p_{21} & p_{22} & p_{23} & \ldots & p_{2, n_{\text {comp }}} \\
\vdots & \vdots & \vdots & \ddots & \vdots \\
p_{d 1} & p_{d 2} & p_{d 3} & \ldots & p_{d, n_{\text {comp }}}
\end{array}\right] \\
=>P C A^{\sigma^{x} T} * m=L Q N^{p}=\left[\begin{array}{c}
l_{1} \\
l_{2} \\
\vdots \\
l_{n c o m p}
\end{array}\right] \\
=>m_{L S}=\left(P C A^{\sigma^{x} T}\right)^{-1} * L Q N^{p}=\left[\begin{array}{l}
m_{1} \\
m_{2} \\
\vdots \\
m_{d}
\end{array}\right]
\end{gathered}
$$

Figure 4.1.2 : Estimation of least squares factor $\left(m_{L S}\right)$ from Kalman Filter predictions

1. As shown in Figure 4.1.2, a measurement of operating system metrics is made (stored as the Kalman filter state $\mathrm{x}_{\mathrm{k}}$ ), and the covariance matrix of this prediction is calculated as $\boldsymbol{\sigma}^{\mathrm{xk}}$. 
2. The principal component matrix $\mathrm{PCA}^{\sigma \mathrm{x}}$ is then calculated for $\boldsymbol{\sigma}^{\mathrm{xk}}$ via eigenvalue decomposition, with $\mathrm{n}_{\text {comp }}$ linearly uncorrelated principal components, where each component is an eigenvector of the covariance matrix $\boldsymbol{\sigma}^{\mathrm{xk}}$

3. $\mathrm{m}_{\mathrm{LS}}$ is then calculated and stored (for future use) as the linear system least squares solution to the system $\mathrm{PCA}^{\sigma \times \mathrm{T}} * \mathrm{~m}=\mathrm{LQN}^{\mathrm{P}}$

4. As shown in Figure 4.1.2.0, when a Kalman filter state prediction is made as $\mathrm{x}_{\mathrm{k}-1}$, the application variables (modelled as $\mathrm{LQN}^{\mathrm{P}}$ ) can be inferred by multiplying the transpose of the principal components matrix by the previously calculated $(\&$ stored) application state factor $\mathrm{m}_{\mathrm{LS}}$, as shown in step 3 (where $\mathrm{m}=\mathrm{m}_{\mathrm{LS}}$ ).

$\begin{aligned} \text { Given } x_{k-1}= & {\left[\begin{array}{ccccc}x_{11} & x_{12} & x_{13} & \ldots & x_{1 n} \\ x_{21} & x_{22} & x_{23} & \ldots & x_{2 n} \\ \ldots & & & & \\ x_{d 1} & x_{d 2} & x_{d 3} & \ldots & x_{d n}\end{array}\right] } \\ =>P C A^{\sigma^{x_{k-1}}}= & {\left[\begin{array}{ccccc}p_{11} & p_{12} & p_{13} & \ldots & p_{1, n_{\text {comp }}} \\ p_{21} & p_{22} & p_{23} & \ldots & p_{2, n_{\text {comp }}} \\ \vdots & \vdots & \vdots & \ddots & \vdots \\ p_{d 1} & p_{d 2} & p_{d 3} & \ldots & p_{d, n_{\text {comp }}}\end{array}\right] } \\ =>L Q N_{k-1}= & {\left[\begin{array}{c}l_{1} \\ l_{2} \\ \vdots \\ l_{n c o m p}\end{array}\right]=P C A^{\sigma^{x_{k-1}} T} * m_{L S} }\end{aligned}$

Figure 4.1.3 : Inferring $L Q N$ parameters $\left(L Q N_{k-1}\right)$ from Kalman Filter state prior 


\section{Chapter 5: Project Description}

This is a research project studying the use of machine learning to tune Kalman filters employed in cloud controllers. Measurement and process noise parameters for the Extended Kalman Filter (EKF) [8] are typically selected by hand and distributed together with the filter once desirable performance is achieved during design testing. This approach produces tracking devices that work well enough for ideal conditions, but that can perform poorly for less optimal conditions, or unforeseen environments.

In order to provide strong performance in all environments where the Kalman filter devices are used, a learning approach for parameter specification is better. This project attempts to accomplish and study that goal by using Long Short Term Memory (LSTM) neural nets to estimate channel and noise covariance matrices. It also learns system process models to estimate the Kalman filter state transition matrix. This method is applied to designing an adaptive controller for performance management of cloud systems. The system utilizes the above techniques for predicting workload states and resource utilizations, for use in the realm of system architecture, design and DevOps [9].

\subsection{Project Organization}

The project is organized into four subprojects: 
1. PCA Layered Queueing Network (LQN) Parameter Inference Algorithm in chapter 4 . This covers the detailed steps of the algorithm, including the relevant math behind PCA. There is also the dimensionality reduction algorithm which is outlined in section 8.6.

2. The controller and its components in section 6.1.

a. LSTM Unit for learning short and long term prediction system measurements, and tuning Kalman filter input parameters in section 6.1.2

b. EKF Unit for tracking system measurements, inferring of application state, and making predictions about future system state in section 6.1.3

c. Control Unit for computation of control actions and orchestrating application and resource instances in section 6.1.4. It also interacts with the operating system, monitored application and its resource

d. User-specified application model and configuration file format and samples in section 6.1.5

3. The controller testbed and its components in section 6.1

4. The LSTM \& Kalman filtering testbed for model tracking in section 6.2

5. There is a system-wide component-based analysis, including related algorithms like KF-PCA in chapter 8

6. An overall black box controller system testbed-based analysis is in chapter 9 


\section{Chapter 6: Control System \& Experimental Testbeds}

This chapter covers the implementation details of the control system itself, and the testbeds used to evaluate it and its components. The testbeds include the control system testbed and the performance model tracking testbed.

\subsection{Control System Architecture}

\subsubsection{Control System Overview}

The control system consists of the LSTM learning unit, Extended Kalman Filter (EKF) unit, Application unit and Control unit.

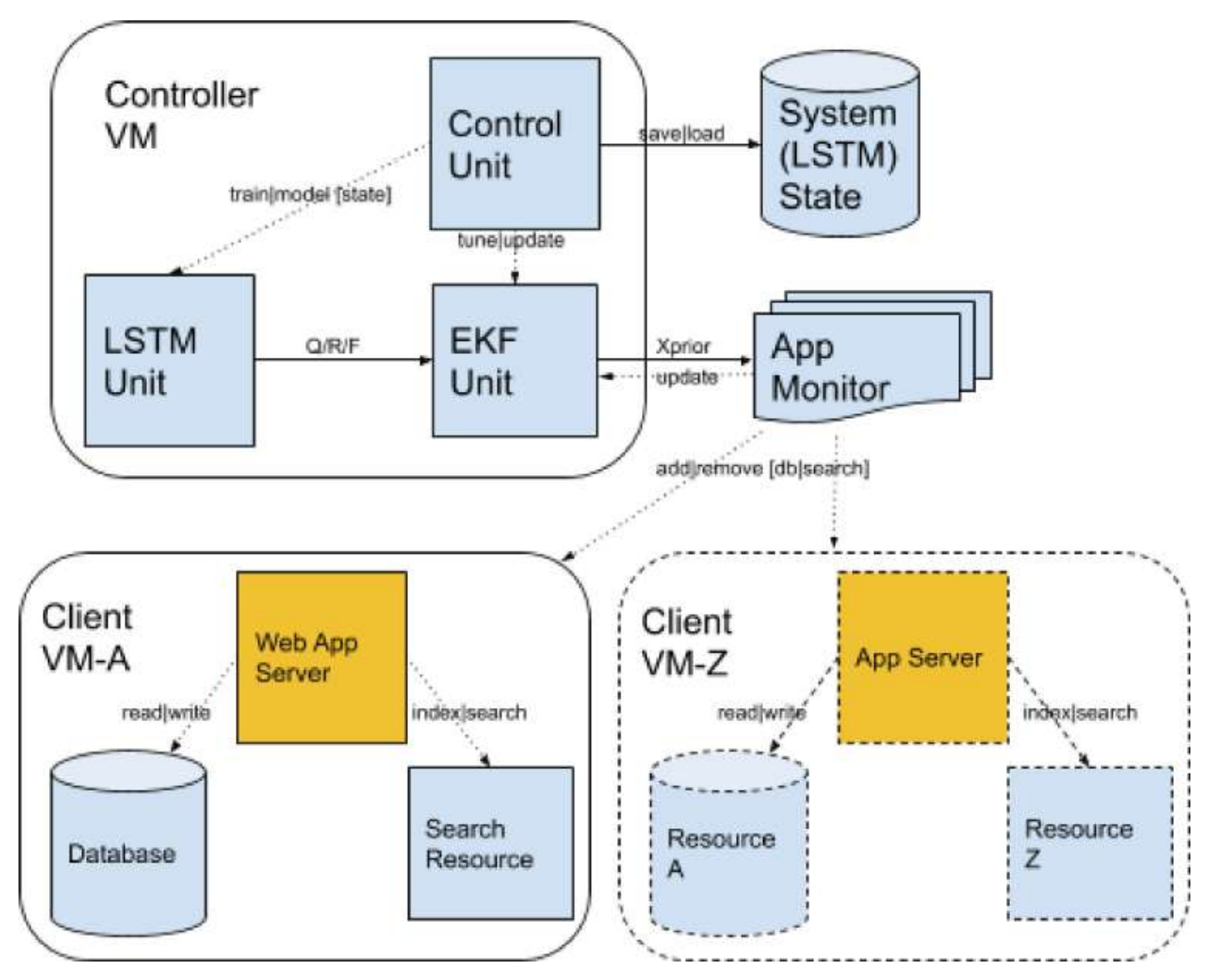

Figure 6.1.1: Control System Architecture 
Table 6.1.1: Output of Linux Top Command

\begin{tabular}{|r|l|r|r|r|r|r|r|r|r|l|l|}
\hline PID & USER & PR & NI & VIRT & RES & SHR & S & $\%$ CPU & $\% M E M$ & TIME+ & COMMAND \\
\hline 2368 & root & 20 & 0 & 92900 & 2108 & 1900 & S & 0.3 & 0.1 & $0: 40.45$ & irqbalance \\
\hline 1 root & 20 & 0 & 19688 & 2500 & 2172 & $S$ & 0 & 0.1 & $0: 01.92$ & init \\
\hline 2 root & 20 & 0 & 0 & 0 & 0 & $S$ & 0 & 0 & $0: 00.00$ & kthreadd \\
\hline
\end{tabular}

The LSTM unit is prompted by the train \& model commands to take in operating system (OS) performance measurements (top 3 processes from the 'top' linux command - see table 6.1.1), encodes them to neural network inputs and produces EKF state model (F), process noise $(\mathrm{Q})$ and measurement noise $(\mathrm{R})$ coefficient matrix estimates. It calculates the EKF state model $(\mathrm{F})$ in case the performance measurement variables are autoregressive rather than random walks.

N.B: The top 3 processes are used in terms of default sort order due to tuning done to determine the optimal input dimension in terms of the GPU resources available for this experiment.

The EKF unit is prompted by the update command to first predict the next measurements $\left(\mathrm{x}_{\mathrm{k}-1}\right)$ and then update its state $\left(\mathrm{x}_{\mathrm{k}}\right)$ using the current Kalman filter measurement value $\left(\mathrm{z}_{\mathrm{k}}\right)$. The Control unit consists of the main controller thread, plus the application monitors in separate threads. Each monitor manages interaction between the main control unit thread, the application model (Layered Queueing Network - LQN), system resources and the application itself. It processes the operating system measurement predictions $\left(\mathrm{x}_{\mathrm{k}-1}\right)$ using the KF-PCA algorithm to predict information about the LQN model, regarding 
utilizations and demands (LQN parameters). It executes model-based scenarios to drive provisioning actions (add or remove) on the monitored application.

The Control unit manages the lifecycle of the various components. It orchestrates the persistence of controller state, the train \& model calls to the LSTM unit, tune and update calls to the EKF unit coordinates the flow of data between the controller's components. It also interacts with the OS regarding taking in performance measurements, and collecting and reporting results throughout operation.

\subsubsection{Software Requirements}

1. The testbed and controller were implemented on an Amazon Web Services (AWS) image. The specific image used was the Deep Learning AMI Version 11.0 image for Ubuntu. It is a tier 2 medium system with 2 vCPUs, and comes with Python pre-installed. These are available on the free tier of compute services, despite being designed for industry-standard compute workloads, hence their appeal for research projects.

2. Install Pip, Tensorflow \& Filterpy Python libraries.

3. Install Wine 2.0 or higher to run the LQN solver [1]

\subsubsection{Learning (LSTM) Unit}

This unit orchestrates the receives operating system measurement metrics and controller state maps. It produces a trained LSTM model and Q, R and F coefficient matrices. 
Estimation of $\mathrm{F}$ is not strictly necessary, but is added as extra functionality, primarily for purposes of analysis, and to verify the correctness of the tuning algorithm.

There are 72 LSTM cells structured as shown in section 2.2 that make up the LSTM Unit. The unit itself is shown in illustration 6.1.2.0.1 as shown below.

An array of these LSTM cells (Section 2.2) are employed with weighting and biasing in order to provide an expandable LSTM unit as shown below. The weight and bias values are to be analyzed iteratively as the LSTM unit runs. The weight and bias connections are shown below. Where:

$\mathrm{msmts}_{\mathrm{k}}=$ vector of 8 normalized measurements from the 'top' 3 processes at time step $\mathrm{k}$ $\mathrm{I}=\mathrm{LSTM}$ input $=\left\{\mathrm{msmts}_{\mathrm{k}} \mathrm{Umsmts} \mathrm{k}-1_{1} \mathrm{Umsmts}_{\mathrm{k}-2}\right\}$,

$\mathrm{O}=$ LSTM output

$\mathrm{n}=|\mathrm{I}|=|\mathrm{O}|=3 \times 24=72$,

$\mathrm{Q}=\mathrm{O}[0: 23], \quad \mathrm{R}=\mathrm{O}[24: 47], \quad \mathrm{F}=\mathrm{O}[48: 72]$ 


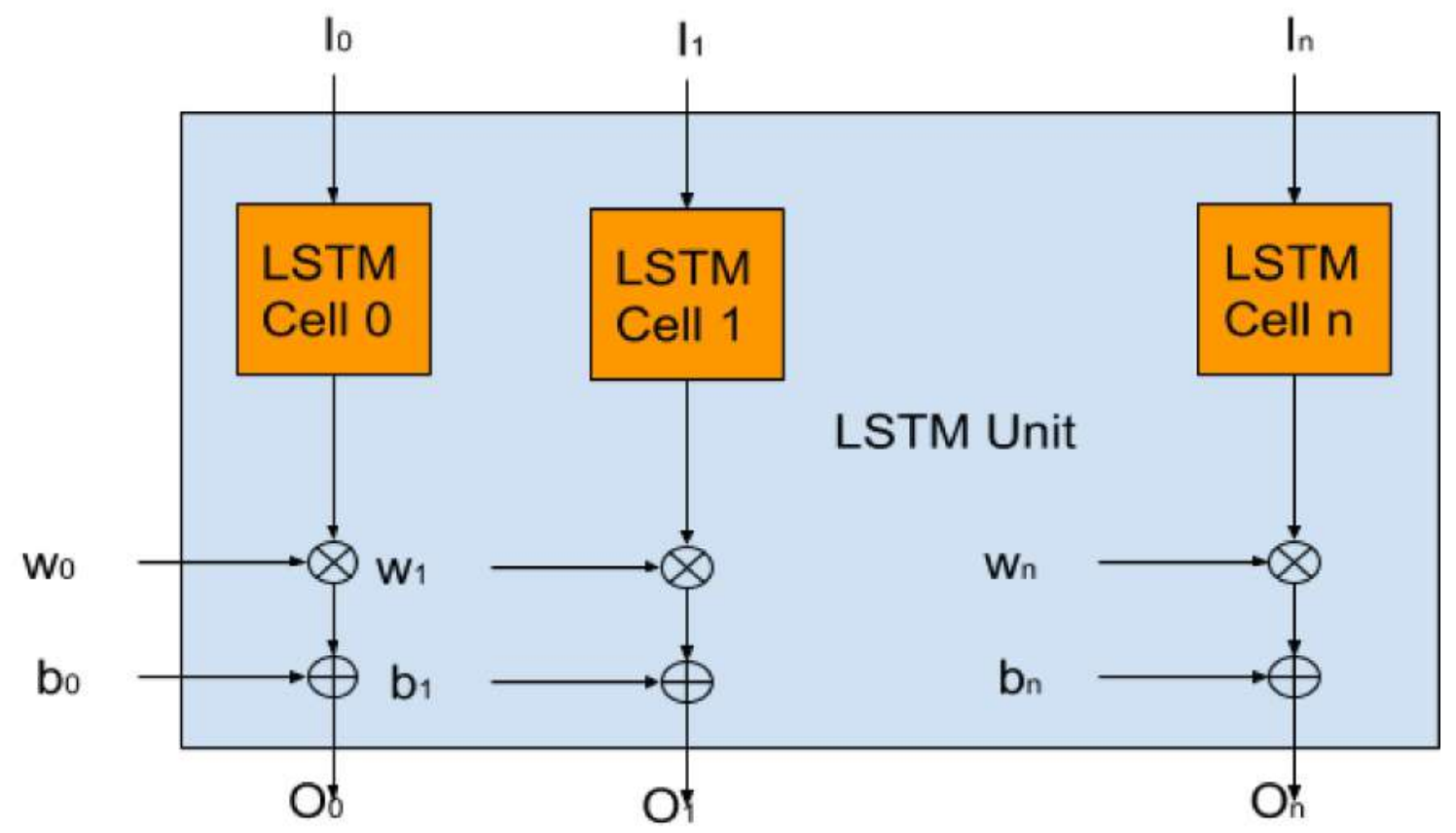

Illustration 6.1.2.0.1: LSTM Unit

\subsubsection{LSTM Unit Logistic Regression Encoding \& Decoding}

For softmax used in this logistic regression setup to work, there needs to be encoding of the input data from real number measurement values to categorical features.

softmax, $\sigma_{z j}=\frac{e^{z(j)}}{\Sigma_{k=1}^{K} e^{z(k)}}$

where $z(j) \& z(k)$ are the output and the label coefficients

To do this, first the values are normalized to the $[-1,1]$ range using $Z^{\prime}(k)=\operatorname{erf}(\mathbf{z}(\mathbf{k}) / \mathbf{N}(\mathbf{k}))$ where $\mathrm{N}(\mathrm{k})$ is the normalization factor as described in Section 7.2.1. The values vector 
$\mathrm{Z}^{\prime}(\mathrm{k})$ is then multiplied by the number of features, $\mathrm{n} \_$features, and used to index the identity matrix $\mathbf{I}_{\mathbf{n} \_ \text {features }}$ to get a set of 'one-hot' vectors.

N.B: A 'one-hot' vector is a feature vector with a single 1 for the indicated feature's index and zeros for the remaining indices.

$$
z^{e}(k)=I_{n \_ \text {features }}\left[\operatorname{erf}(z(k) / N(k)) * n_{-} \text {features }\right](7)
$$

with each entry representing a feature. Encoding of coefficient label values is equivalent to that of measurement values, with the number of output classes, n_classes in place of $\mathrm{n} \_$features and $\mathrm{z}(\mathrm{k})$ replaced by the coefficient value $\mathrm{c}_{\mathrm{k}}$

$$
c^{e}(k)=I_{n \_ \text {classes }}\left[\operatorname{erf}(c(k) / N(k)) * n \_ \text {classes }\right](8)
$$

Conceptually speaking each feature and class represents sub-range of the $[-1,1] \operatorname{erf}(\mathrm{x})$ error function domain.

The decoding procedure is the reverse of encoding, using the inverse error function and normalization factors,

$$
z(k)=N(k) * e r f^{-1}\left(z^{e}(k) \text {. range }\left(n_{\text {features }}\right) / n_{\text {features }}\right) * n_{\text {features }}(9)
$$

where range(n_features) is the vector of increasing column indices

$$
\operatorname{range}(k)=\left[\begin{array}{c}
1 \\
2 \\
\cdots \\
k
\end{array}\right]
$$

and for coefficient one-hot vectors

$$
c(k)=N(k) * \operatorname{erf}^{-1}\left(c^{e}(k) . \text { range }\left(n_{\text {classes }}\right) / n_{\text {classes }}\right) * n_{\text {classes }}(10)
$$




\subsubsection{LSTM Parameters}

There are two methodologies followed in learning the model parameters, and these are reflected in two types of LSTM units. One uses Linear Regression with Gradient Descent optimization (KF-LSTM1). The other uses classification via Logistic Regression (KF-LSTM2). These two variants are supported and fully implemented, though KF-LSTM1 is used by default.

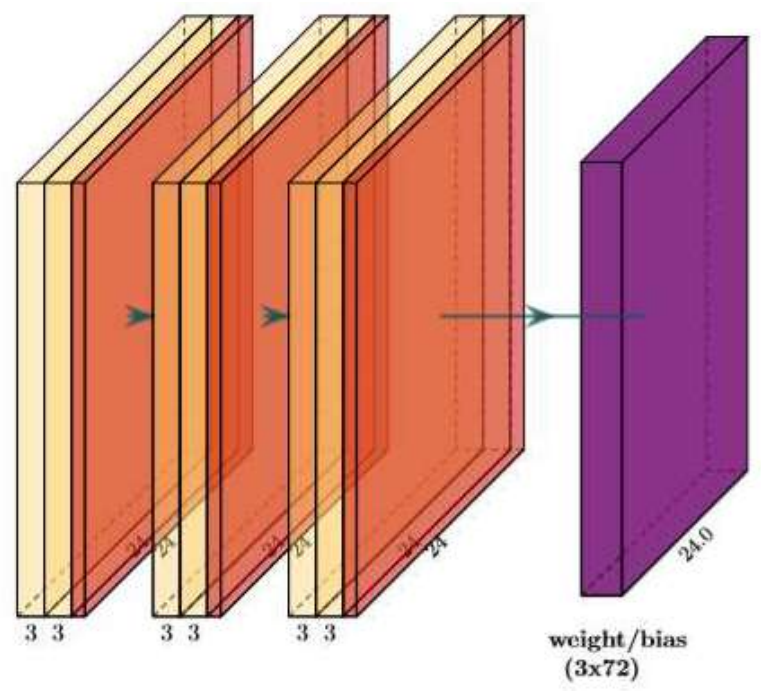

Figure 6.1.2.2: Linear Regression LSTM-cell static RNN

For linear regression, a static RNN network is used with an input size of [n_entries, n_msmts] where $n \_$entries $=3$ (number of time steps in the past) and $n \_m s m t s=24$ (number of measurement values parsed from the linux 'top' command - see table 6.1.1).

\begin{tabular}{|l|l|l|l|l|l|}
\hline $\begin{array}{l}\text { Learning } \\
\text { rate }\end{array}$ & Epochs & $\begin{array}{l}\text { Samples } \\
\text { per epoch }\end{array}$ & $\begin{array}{l}\text { Hidden } \\
\text { layers }\end{array}$ & Cell type & Network type \\
\hline 0.01 & 10 & 10 & 2 & LSTM / MultiRNN & static / dynamic RNN \\
\hline
\end{tabular}


The weights and biases used have dimensions [n_hidden, $\left.n \_l s t m \_o u t\right]$ and [n_entries, n_lstm_out] where n_hidden $=2$, n_entries $=3$ and n_lstm_out $=72$. These are applied to the network and output layer accordingly.

\subsection{Bias Selection, Weights, Cost \& Optimization}

Bias selection is an important part of learning algorithms. In this case, the best results were seen when the bias point was selected from the best label data. The initial training data is sorted based on Kalman filter performance, and the active learning procedure in the following. The corresponding weights are initialized to ones and updated through back-propagation. For logistic regression, the weights are a zero mean normal distribution truncated at two standard deviations.

The cost function used in linear regression is a mean square error reduction (minimizing the difference between output and labels), with gradient descent optimization, specifically using momentum optimization [108] with a momentum factor of 0.9. Logistic regression uses cross entropy mean reduction on the softmax of the model's output as the cost function. It then does a gradient descent optimization [108] to select minima.

\subsubsection{KF-LSTM Accuracy \& Error}

This section describes the tuning of the KF-LSTM. KF-LSTM is used to track performance measurements to predict future values of performance measurements. There are two variants of KF-LSTM based on the type of LSTM: regression-based KF-LSTM1 and classification-based KF-LSTM2. 


\subsection{Calculation of LSTM (KF-LSTM1) Regression Prediction Error}

This section defines the prediction error of KF-LSTM1. Error is used because it is the proper measure for linear regression training, since classification accuracy is not defined on this type of LSTM network as labels are real numbers rather than classes.

The training cost function is defined as the mean square difference between the model output and label data. This is calculated using Tensorflow [109] during the training phase. In the testing phase, the mean squared error between the LSTM output layer (predicted coefficients) and labels (best coefficients from prior iterations) is used to determine the cost (see below).

Where $\mathrm{N}$ is the number of training labels, $\mathrm{C}(\mathrm{k})$ is the predicted coefficient, and $\mathrm{C}^{\mathrm{A}}(\mathrm{k})$ is the actual coefficient for label $\mathrm{k}$ :

$M S E, \varepsilon=\frac{\sum_{k=1}^{N}\left(C(k)-C^{A}(k)\right)^{2}}{N}$.

NB: MSE is calculated from LSTM output (real number prediction score $\mathrm{S}(\mathrm{k})$ ) and the labeled optimal coefficient (real number coefficient $\mathrm{C}(\mathrm{k})$ ) over $\mathrm{N}$ coefficients as:

$$
\frac{\sum_{k=1}^{N}(S(k)-C(k))^{2}}{N} .
$$

\subsection{Calculation of LSTM (KF-LSTM2) Classification Prediction}

\section{Accuracy}


This section defines the classification accuracy of the KF-LSTM2.

The training cost function is defined as the mean square difference between the model parameters and label data. This is calculated using Tensorflow [109] during the training phase.

In the testing phase, the accuracy is determined as the mean number of correct predictions in the output. These predictions are produced through softmax, so add up to a maximum accuracy of 1 for a perfectly matching output. To produce them, the output tensor [109] of the LSTM model is passed through softmax, and a correct prediction is determined as equality between the largest argument vectors of the output and label tensors/vectors.

A summary of the above algorithm is shown below:

softmax, $\sigma_{z j}=\frac{e^{z(j)}}{\sum_{k=1}^{K} e^{z(k)}} \quad[13]$, where $\mathrm{z}(\mathrm{j}) \& \mathrm{z}(\mathrm{k})$ are the output and the label

coefficients

correct prediction, $P^{C}(i)=1-\left|\arg \max \left(\sigma_{z}(M)\right)-\arg \max (Y)\right|$

$\operatorname{LSTM}$ Accuracy $=\sum_{i=1}^{N^{P C}} \frac{P^{C}(i)}{N^{P C}}$

where $i$ is the index of each entry in the output tensor, M is the LSTM model output, Y is the test label tensor, $\mathrm{N}^{\mathrm{PC}}$ is the number of entries in the output tensor (and label tensor) 


\subsubsection{KF-LSTM Data Acquisition - Active Learning}

Data acquisition by active learning, employed here as an iterative approach to training the

\section{LSTM.}

Active learning [3] is a type of machine learning where greater accuracy can be achieved with few or no training labels if the algorithm can choose its own training data. It sends queries in the form of unlabeled data to the labeling module, commonly called an oracle [3], for labeling.

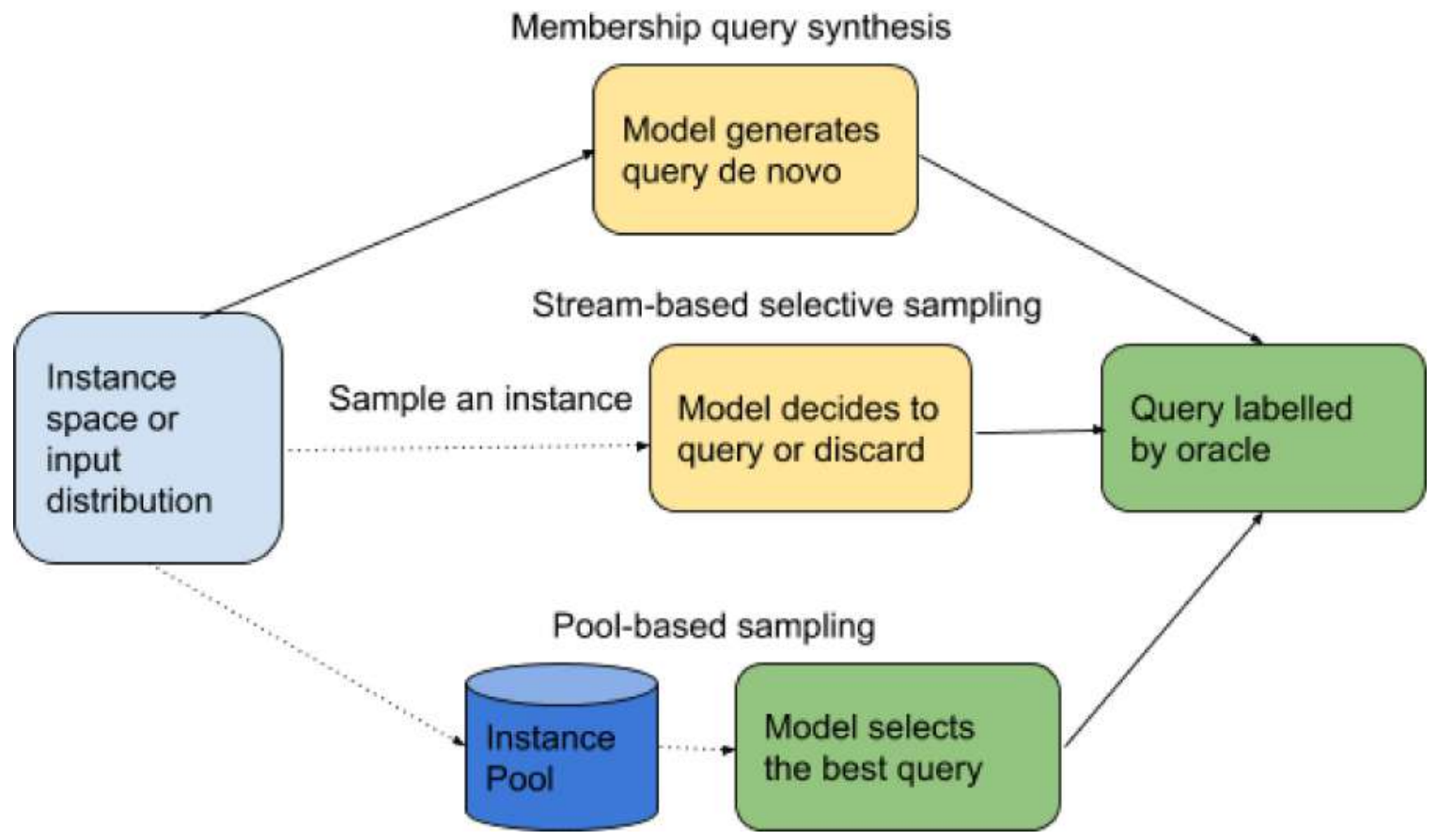

Illustration 6.1.2.4: Three main active learning scenarios ${ }^{[3]}$

The following steps describe the process: 
1. In the first iteration, a batch of random coefficients are used to initialize the Extended Kalman Filter (EKF)

a. The performance of the EKF by prediction accuracy is then calculated for each set in the batch, and the top performing sets from the batch are selected.

2. In the next iteration, the selected coefficient sets and their corresponding measurements are used as labels for training the LSTM. The trained network is then used to predict a new batch which is then analyzed in the first iteration, and a new subset selected.

3. It is expected that with each iteration the accuracy of the EKF will improve provided the measurements are consistently rich. Perturbations in the system should cause dips in accuracy as the network learns the characteristics of the new measurements. After a sufficient number of iterations, the system could be said to be well tuned. Pseudocode for the algorithm can be seen below:

\# Comments are indicated using the left most '\#' has symbol on each line \# Summary: Return highest accuracy LSTM-predicted coefficients as labels \# $\quad$ To be used in bootstrapping (training) the LSTM unit \# Generates model training labels by a bootstrap active-learning approach def bootstrap_labels(model, X, sample_size $=5$, action_interval $=40$ ):

(labels, ekf, msmts, history, pred_per_sample $)=([]$, None, [], [], 10) $($ accuracies, minimum_accuracy $)=([], 0.7)$ 


$$
\begin{aligned}
& \text { for } \mathrm{i} \text { in range(0, sample_size): \# Loop through all samples } \\
& \text { new_msmts }=\text { measurements }() \quad \text { \# Get new measurements } \\
& \text { if len(msmts): } \\
& \text { (best_coeffs, best_accuracy) }=([], 0) \\
& \text { for j in range(0, pred_per_sample): \# Repeat for all predictions } \\
& \text { coeffs }=\text { predict_coeffs }(\text { model }, \text { msmts }, X) \quad \text { \# Predict coefficients } \\
& \text { logger.info("pre build_ekf.coeffs }=\text { " + str(coeffs)) \# Log coefficients } \\
& \text { ekf = build_ekf(coeffs, [msmts]) \# build new EKF } \\
& \text { accs, accuracy }=\text { ekf_accuracies(ekf, new_msmts) \# calculate EKF accuracy } \\
& \text { if accuracy }>=\max ([\text { best_accuracy, minimum_accuracy]): \# if best accuracy } \\
& \text { best_coeffs = to_size(coeffs, 1, n_coeff) \# resize coefficients } \\
& \text { best_accuracy }=\text { accuracy \# update the best accuracy } \\
& \text { accuracies.append( [accs, best_accuracy]) \# extend the accuracies list } \\
& \text { if len(best_coeffs): \# if any good coefficients available } \\
& \text { labels.append([to_size(msmts, n_msmt, n_entries), best_coeffs]) \# add a label } \\
& \text { sleep }(0.5) \quad \text { \# Reduce load on system resources } \\
& \text { if } \mathrm{i} \% \text { action_interval }==0 \text { : \# Every 'action_interval' intervals } \\
& \text { do_action(ekf, msmts) \# Controller actions from predictions } \\
& \text { msmts }=\text { new_msmts } \quad \text { \# Save new measurements } \\
& \text { history.append(msmts) \# \# Update measurement history } \\
& \text { return labels \# Return the created labels }
\end{aligned}
$$

Figure 6.1.2.5: Active learning algorithm for bootstrapping LSTM training labels

\subsubsection{EKF Unit}

The Extended Kalman Filter unit consists of an EKF as described in Section 2.3. 


\subsubsection{Measured Variables \& Normalization Factors}

The EKF unit takes as inputs measurements of system utilization in the form of the top linux processes (from the "top" command in this implementation). On Ubuntu this yields the process identifier (PID), priority (PR), time running niced processes as a percentage (NI), virtual memory in Kb (VIRT), resident memory size in Kb (RES), shared memory size in $\mathrm{Kb}(\mathrm{SHR})$, central processing unit utilization (\%CPU), physical memory utilization (\%MEM), and CPU time in hundredths of a second (TIME+) [13].

The normalization factors for each measurement variable are shown in Figure 6.1.3.1.

Figure 6.1.3.1: Measurement Normalization Factors

N.B: Measurement normalization is calculated as: $\widehat{z}(i)=\frac{z(i)}{N(i)}$ for each process

\begin{tabular}{|l|l|l|l|l|l|l|l|}
\hline N(PR) & N(NI) & N(VIRT) & N(RES) & N(SHR) & N(CPU) & N(MEM) & N(TIME) \\
\hline 40 & 1 & $10^{9}$ & $10^{9}$ & $10^{9}$ & 1 & 1 & $10^{6}$ \\
\hline
\end{tabular}




\subsubsection{State Variables}

As shown in the section above, the EKF unit takes as inputs measurements of system utilization in the form of the top linux processes. The state variables are then taken to be the same quantities (and units) as the measured variables, with a one state variable per measurement. The state prediction is then interpreted as a direct prediction of the next measurement value.

The set state equations used by the Extended Kalman Filter are covered in Section 2.3, and in Appendix A.

\subsubsection{Process Noise, Measurement Noise \& State Transition Matrices}

Typically zero and identity matrices can be used as default values for $\mathrm{Q}, \mathrm{R}$ and $\mathrm{F}$ matrices of the Kalman Filter. When system dynamics are known or can be inferred, such as using the active learning approach, higher performance can be achieved by the tuned Kalman Filter. The use of LSTM for learning Q, R and F matrices to tune the Kalman filter is referred to as the KF-LSTM algorithm.

All the matrices converged after a few iterations, and a significant boost was seen in the Kalman Filter's prediction accuracy (section 8.3), in cases where the operational data is similar to the training data, i.e. high application activity during both tuning and operation, or low application activity seen in both modes.

Two matrix layouts were considered: diagonal matrices and full symmetric matrices. This was done to meet requirements and avoid errors during the update and prediction 
operations of the filter. Both layouts were tested with the LSTM unit learning the matrix coefficients and updating the filter in turn, and there was minimal difference in terms of performance. Before settling upon fully symmetric matrices, random full matrices were considered. These would occasionally produce invertibility errors, but overall gave equally stable Kalman Filters. Due to this invertibility issue, it was preferable to ensure full matrices were symmetric.

For a description of the best performing state transition $(\mathrm{F})$ matrix, please see the features section of the LSTM unit above.

\subsection{Coefficient Selection For Q, R \& F Matrices}

The LSTM unit attempts to learn the values for the Q, R and F matrices. Various estimation methods are used to add features to the training data for these coefficients. For the $\mathrm{Q}$ and $\mathrm{R}$ noise matrices, an approximation of the autocovariance least squares [101] method is used. In this case, the time delayed autocovariance of the Kalman filter innovations (Section 2.4 eq (3)) are added as feature values for the LSTM. The time delay values are an increasing series up to a user-specified maximum.

For the F state transition matrix, values chosen are the ratios between the current and previous state. Again, noise estimation is also done here to determine the relevant covariance values.

All possible $\mathrm{Q}, \mathrm{R}$ and $\mathrm{F}$ matrix combinations are compiled and evaluated to determine their utility through average filter error. The best performing combinations are retained as features and class labels for the LSTM unit's training and test data. 


\subsubsection{Control Unit}

The Control Unit (see figure 6.1.1) manages the flow of information in the control system and its components. It ensures the flow of data through the system, through these functions:

1. Reading and encoding performance measurement data from the operating system into tensor [109] variables. This is done in a loop with a delay of less than $10 \mathrm{~ms}$ between readings on average, although this duration depends on the specific runtime environment

2. Feeding input tensor variables to the LSTM unit in the tuning and operational phases.

3. Decoding LSTM output and updating the EKF unit during tuning and operation

4. Feeding EKF output to the App unit during the operation phase to drive optimization actions

5. Saving and loading the LSTM unit state to/from persistence (the file system)

There are two modes of operations of the Control unit, determined by user input:

1. Tuning mode, which involves training and testing the LSTM unit and initializing the EKF unit

2. Control mode, which involves taking in operating system real time data, encoding and passing it to the EKF unit and Application unit (in the next section) 


\subsubsection{Monitors}

The control unit in operational mode consists of a set of host monitors managing application components and resources on separate hosts. These monitors run on dedicated threads continually taking operating system measurements and executing control actions for optimal performance. From the system overview diagram in section 6.1 .1 , they issue the add \& remove (database \& search engine) operations using operating system metric predictions $\left(\mathrm{X}_{\mathrm{k}-1}\right)$ to make model driven decisions about which resources to provision.

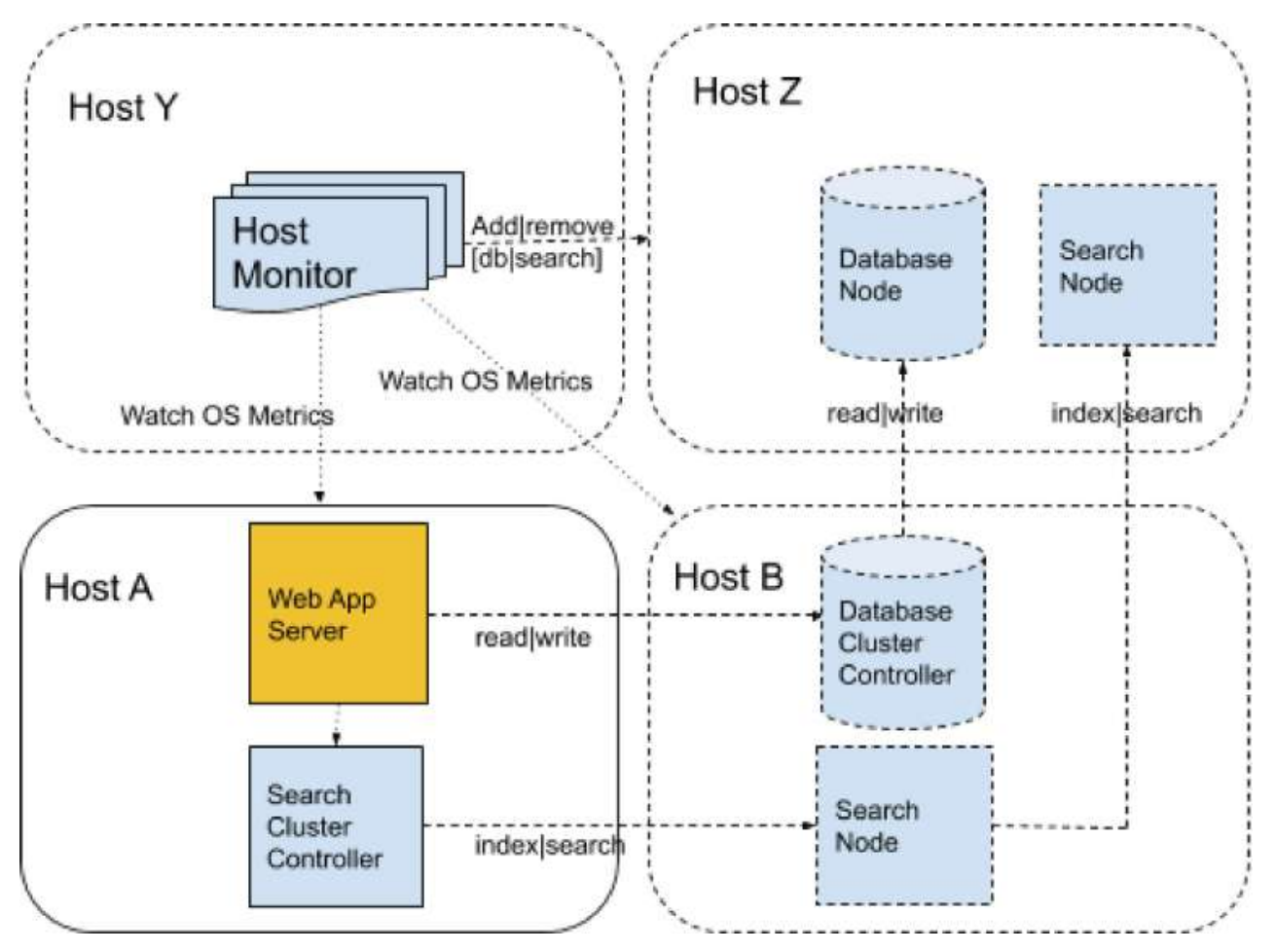

Figure 6.1.4.1: Host Monitors, Application \& Resource Control

Each monitor operates in two modes: tuning mode and control mode. 
Tuning mode happens just after creation of the monitor thread. The training of the LSTM unit for EKF coefficient discovery is done using measurements from the newly enrolled host. The measurements, coefficients and linear measurements-to-LQN model are determined and saved to system state, and persisted in the state repository.

Control mode involves taking measurements from the host via configured commands and making predictions about future resource state using LQN model-based reasoning. Using these predictions or the current measurements, and resource thresholds, actions may be then issued by each host monitor to scale up (or down) the affected host resource. Monitors persist in control mode until the end of the controller's life cycle, at which point all the threads are shut down, and metric data compressed into host-specific tarball archives.

There are two types of control mode: predictive and non-predictive control. Predictive control is control mode using predictions of future measurements (from the Kalman filter), while non-predictive control is control mode using current measurements. Configuration options to choose between the two control mode types are supported (see Section 6.1.5). Predictive control is useful when avoiding potential performance degradation is important. Non-predictive control is geared towards mitigating existing performance issues, and also in scenarios where assessing the impact of the measurement predictions is desired. Active control refers to control mode where actions are issued by the host monitors to scale up/down resources. Passive control refers to control mode where no actions are 
issued, the monitor simply collects and logs measurement data. An active monitor is one in active control mode, while a passive monitor is one in passive control mode.

Provisioning actions taken by the monitor are user-specified (configuration parameter 'provision-cmds' \& 'deprovision-cmds' - Section 6.1.5). Provisioning actions are taken when the configured metric thresholds are exceeded ('lqn-thresholds' - Section 6.1.5), and deprovisioning actions are taken when metrics cross below configured thresholds. The controller state of each monitoring step of control mode is stored in the system state (System Overview section) repository if a provisioning or deprovisioning action was taken.

\subsubsection{Control Algorithm}

The control algorithm covers the decision making process for determining control actions to be taken. It is executed by the control unit, in each host monitor thread. It involves the following steps (NB: The issued commands are user-specified, defined in section 6.1.5):

1. Take measurements from target application host using the 'login-<application-host>' and 'memory-cmd-<application-host>' commands, parse and normalize metrics (Section 6.1.3.1) as $\widehat{z}_{k}$

2. Use KF-LSTM1 to predict the next measurements $\widehat{x}_{k \mid k-1}$

3. Run KF-PCA to estimate the model parameters $p$.

4. Update and solve the LQN model with 'model-update-cmd' and 'model-solve-cmd', 
5. Compare user-specified thresholds in 'lqn-thresholds' with LQN model utilization output from 'model-solve-cmd'

6. Select and execute specific 'provision-cmd' for upward threshold crossings, and 'deprovision-cmd' for downward threshold crossings. Start again from step 1).

\subsubsection{Configuration File \& Control Commands}

The user specified control commands are configured using a system configuration file in enhanced YAML format. This format includes user-specified variables which allow for concise command templates which are expandable to accommodate an arbitrary command space on an arbitrary number of applications. A template is shown below:

\# Config file specifying application/domain specific commands and scripts \# Variables are user-defined to be used within the scope of the yaml file only variables:

search-host: []

db-host: []

application-host: 127.0.0.1

lqn-model: '/home/user/downloads/system.lqn'

solr-url: 'https://127.0.0.1:8983/solr'

db-home: '/home/user/downloads/apache-cassandra-3.11.2'

\# Provisioning commands are user-specified name-value pairs.

\# The names are ignored, and values can be lists. 
\# They refer to the monitored application, and are strictly a concern of the user only provision-cmds:

-cmd1: "/<solr-url>/solr/admin/collections?action=ADDREPLICA"

$-\mathrm{cmd} 2$ :

- "sed -i 's/seeds:(.*)/seeds:\$1, <db-host>/' \"<db-home>/conf/cassandra.yaml।" "

- "<db-home $>/$ bin/stop"

- "<db-home $>/$ bin/start"

\# Deprovisioning commands are name-value pairs.

\# The names are ignored, and values can be lists

deprovision-cmds:

-cmd1: "<solr-url>/solr/admin/collections?action=DELETEREPLICA"

-cmd2:

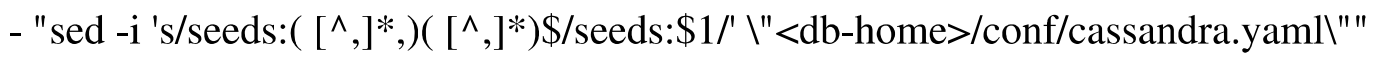

- "<cassandra-home>/bin/stop"

- "<cassandra-home>/bin/start"

\# Logins are required for all monitored application hosts. They have the 'login-' prefix login-<application-host>: "ssh -i 'keypair.pem' ec2-user@<application-host>" login-<db-host>: "ssh -i 'keypair.pem' ec2-user@ < db-host $>$ " login-<search-host>: "ssh -i 'keypair.pem' ec2-user@<search-host>" \# lqn-tasks are tasks in the LQN model referenced in model-update-cmd \# \& model-solve-cmd. They are used to initialize the control unit \# indicating the quantities of model resources in the monitored application 
lqn-tasks:

- nSearch: 0

- nDb: 0

- nWebServers: 1

\# 'upper' or 'lower' thresholds

\# to be crossed when values pass 'upwards' or 'downwards' respectively lqn-thresholds:

- upper: 0.9

- upper: 0.9

- upper: 0.3

\# The memory command is needed for non-linux monitored application hosts memory-cmd-<application-host >: "top -b -n 1 -a"

model-update-cmd: "sed -i 's/<param0> ||$s^{*}=\| l s * \backslash[?[0-9,] \backslash] ? * /<$ param $1>/$ '

$<$ lqn-model $>"$

model-solve-cmd: "wine lqns <lqn-model>"

\section{Illustration 6.1.5: Sample Application Configuration YAML File}

These commands (such as 'model-update-command', 'model-solve-command', etc) are used in the application monitors (Section 6.1.4.1) to interact with the operating system, file system, the monitored application and its resource components. The use of these commands is covered in the control algorithm section.

The application unit ensures that every configuration parameter, including commands, has all variable references resolved. A configuration cache is kept in memory when the application unit starts, so a restart is needed to refresh it. YAML nested data types such as strings, maps and lists are supported. 


\subsubsection{Application Model}

Measurements taken from the operating system state (using the 'top' command) are used by the application host monitor to make inferences about the LQN model parameters of the application under observation. This is done using the output of the Kalman Filter unit $\left(\mathrm{x}_{\mathrm{k}-1}\right)$.

An accurate application model is important for state maintenance, prediction and scenario execution.

A sample layered queueing network (LQN) model is shown below. It references the test client and application described in section 6.3.1. It consists of the test client, application service and backend systems (database and search engine). For more details on the runtime environment and deployment, see section 6.3.1.

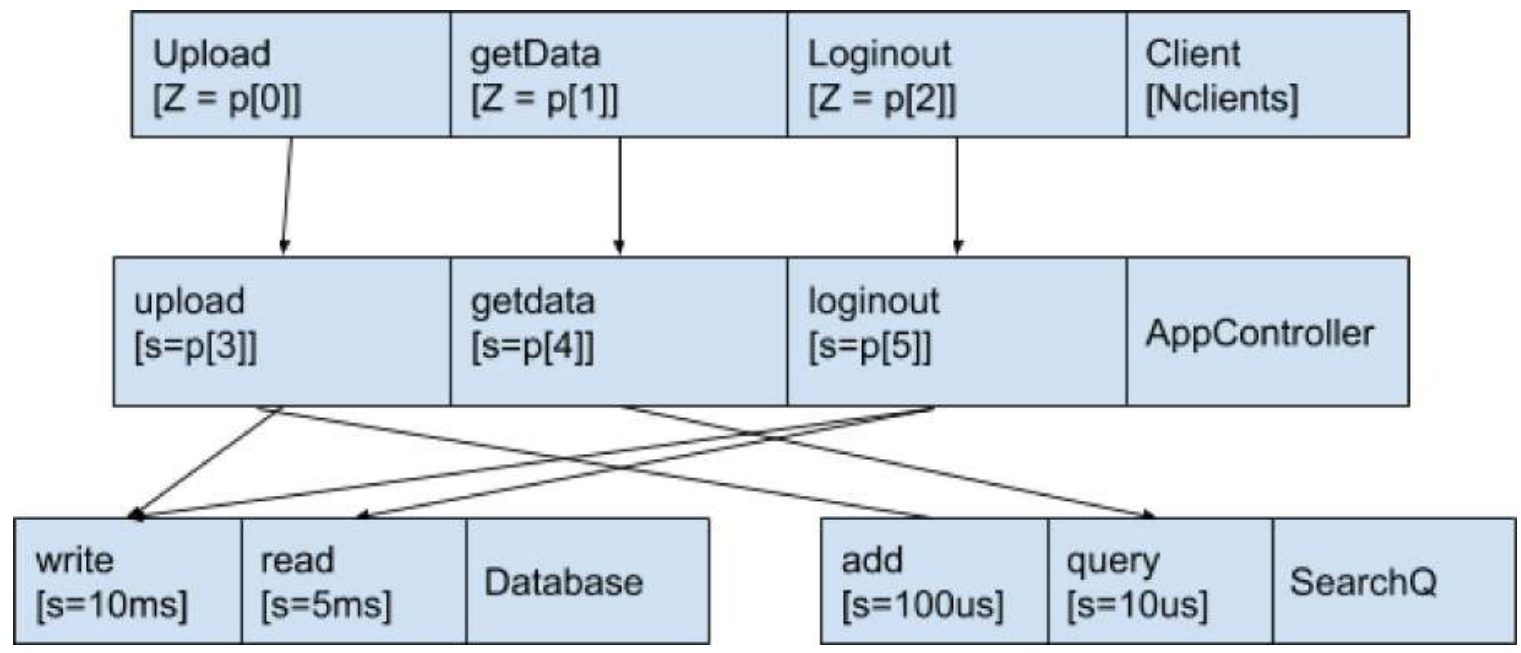

Figure 6.1.5.1: LQN Model of Application System \& Resource Components 


\subsection{Application Model-based Control Behaviour}

In control mode (Section 6.1.4.1), the host monitor will execute the 'model-update-cmd' to set inputs for the LQN model, run the model solver using the 'model-solve-cmd' and read the outputs of the model solver to determine the best scenario to use for the next control action.

The model resource output values associated with that scenario are then used as the desired resource levels for determining if provisioning ('provisioning-cmds') or deprovisioning ('deprovisioning-cmds') actions are to be taken, based on whether the threshold was crossed to a high or low value. Also, the actual provisioning and deprovisioning command executed is quite specific, depending on which threshold was triggered.

\subsection{Control System Testbed}

To accomplish the goal of evaluating a model driven controller for real applications, a controller system testbed was created. This system testbed consists of an instance of the controller, and an application system with associated resources. The controller runs on a dedicated machine, while the application runs on a cluster of virtual machines as shown in Figure 6.2.0. 


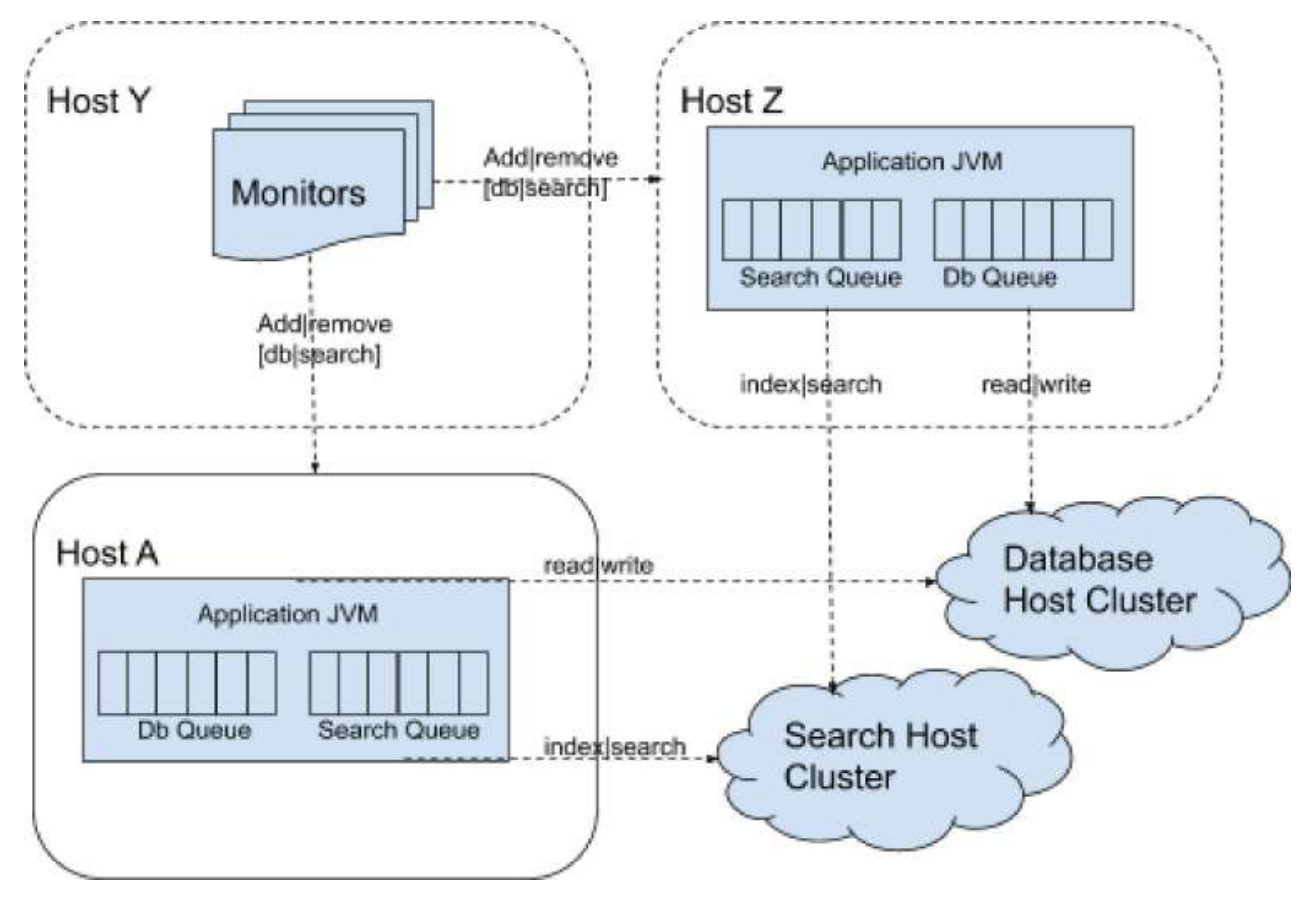

Figure 6.2.0: Testbed System Layout

\subsubsection{Test Application Implementation \& Deployment}

The testbed application was developed in Java for the purpose of investigating the capabilities and potential benefits of the adaptive controller. Various open source tools were used to provide compute and data storage components, and details about the implementation and deployment environment are outlined below.

- The testbed and controller were implemented on an Amazon Web Services (AWS) image. The specific image used was the Deep Learning AMI Version 11.0 image for Ubuntu. It is a tier 2 medium system with 2 vCPUs, and comes with Python pre-installed. Install pip, Tensorflow \& filterpy python libraries. 
- The application JVM is deployed on EC2 compute instances as shown on Figure 6.3.0, running Redhat Linux 7.2. These are tier 2 micro systems with single vCPUs. It is a custom Java application written specifically for the testbed, using the Spring Boot web server consisting of REST controller and session services.

- These services contain two queues, one for database host instances and a second for search host instances, selected via a round robin scheme.

○ The services use SolrJ 6.6.0 and Datastax 3.3.0 clients for search engine and database access.

- The database and search engine applications are running on similar virtual machine hosts to the application JVM.

The database is a Apache Cassandra cluster containing two nodes with only one enrolled at startup. The controller will look to add a new node at runtime when the configured thresholds for resource utilizations and demands (more on this later) are crossed. Similarly, the search engine is an Apache Solr cluster containing two nodes, with only one at startup. A similar provisioning and deprovisioning policy is employed for the search engine via its replica addition and deletion API.

See Section 6.1.5 for examples of the provisioning and deprovisioning scripts used for the monitored resources.

\subsubsection{Testbed Client \& Traffic Generators}


A test client was also scripted to automate the launch of the testbed and generation of client traffic. This was to enable consistency in timing and coordination between different test runs. A summary of the testbed client algorithm is shown in the diagram below:

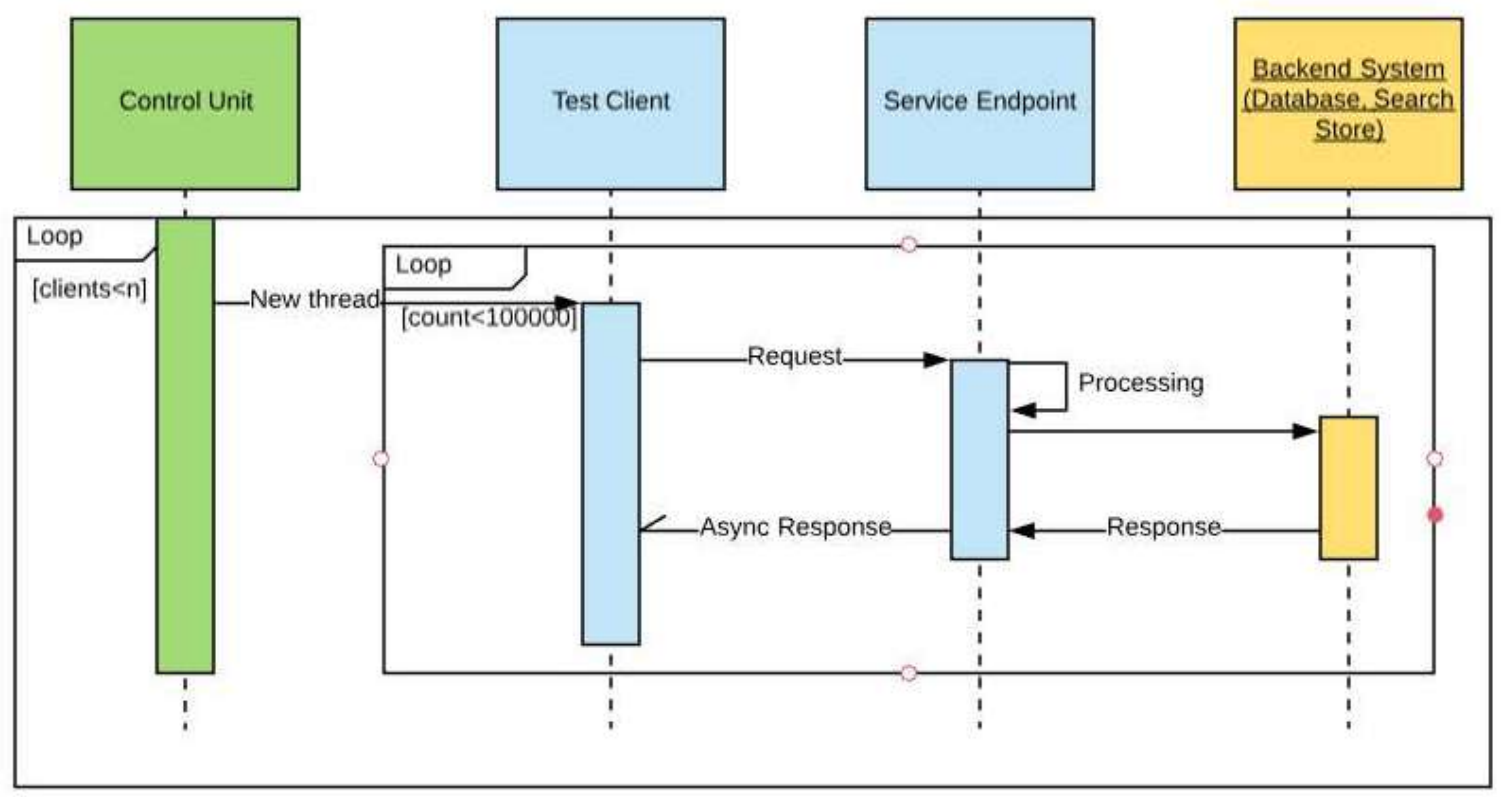

Figure 6.2.2: Test Client Operation Sequence Diagram

Client traffic was produced using a script, with requests arriving as described in section 7.2. This basic traffic generation scheme should ensure a full vertical request trajectory involving the major layer queues in the application model.

It is expected that most traffic requests should have standard service demands in the low millisecond range, but over time latency will accumulate as the service queues fill up, eventually leading to a large drop off if/when the queues are full and service threads start to fill up the threadpool.

This threadpool acts as a second level of queueing which could cause request errors to occur (as HTTP code 500 server errors) and would represent potentially catastrophic 
server failure should the web server not be able to recover from the condition. Mitigating such a scenario is one key motivation for active monitoring, and for this investigation.

\subsection{Performance Model Tracking Testbed}

In the research goal of determining how to model real time applications, tracking these application models through estimation is an important task. To solve the problem of estimating relevant unknown parameters of system performance models, it is useful to track them using hidden parameters [16]. The control system in passive control mode has been used here for its model tracking capability to fulfill this task. Overall the performance model tracking testbed displayed quick convergence to model parameters $p$ from measurement data, and the ability to detect changes in measurement data requiring recalculation of $p$.

To this end a second testbed was created, with the task of estimating parameters in a nonlinear system of known structure. The unknown vector $p$ is a parameter of this system. The Kalman filter $\mathrm{H}_{\mathrm{k}}$ matrix (Section 2.3.1) is the matrix of partial derivatives of measured values (z) with respect to $p$. It can be found by solving the model with $p$ changed for $(\Delta p)$. The operation of the testbed involves two subproblems:

1. The learning problem of converging to unknown $p$ from known system parameters 
2. The tracking problem of detecting changes in the known system parameters and appropriately triggering step 1) above.

A prepared data sequence with parameter and noise drift is tracked by the testbed in order to estimate the underlying model parameters of interest. Various metrics are then collected associated with this tracking method, such as speed of convergence, tracking accuracy and starting point robustness (i.e. consistency from different starting points in the data).

The testbed is also capable of estimating the noise covariance coefficients of the data sequence.

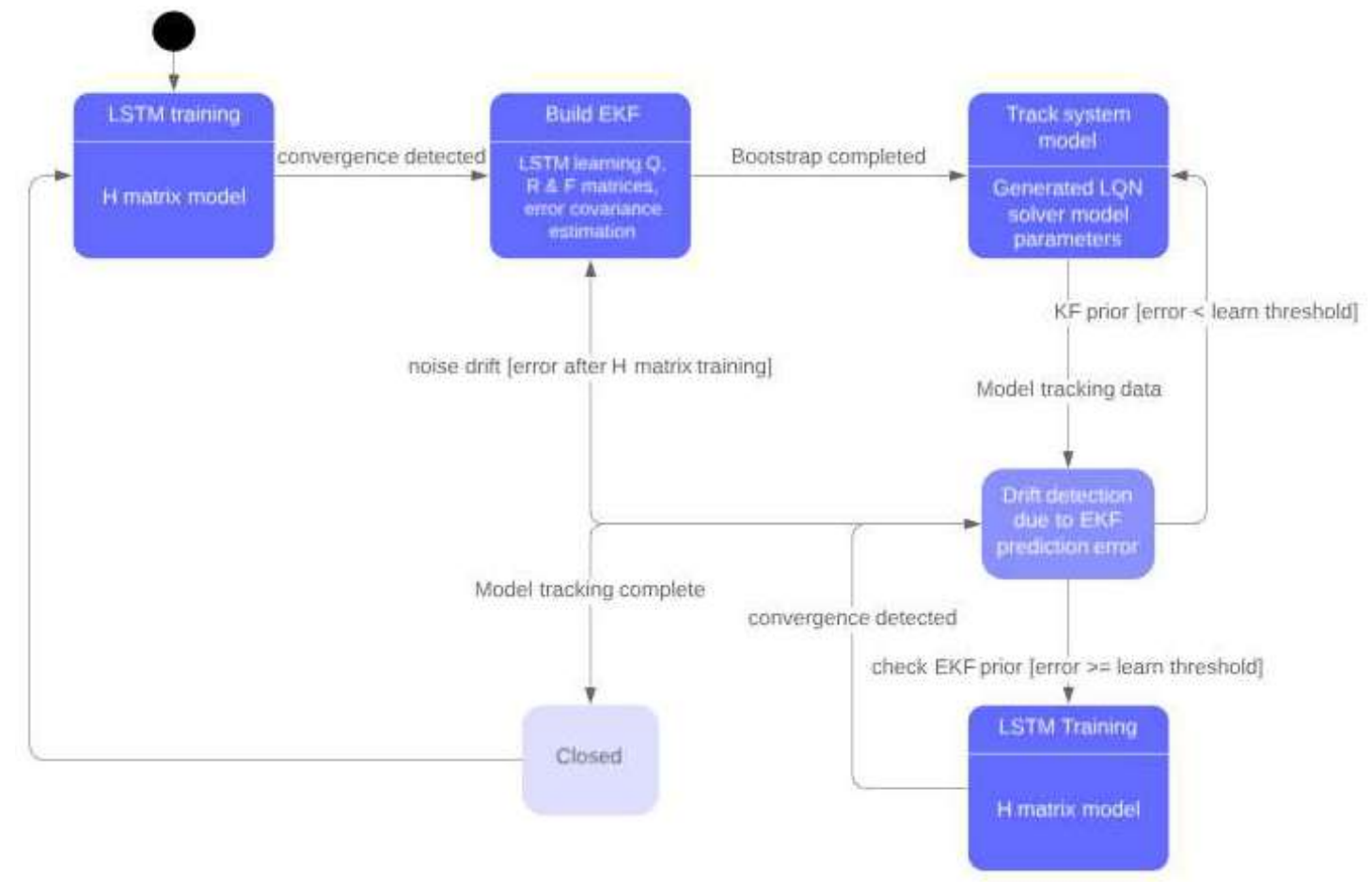

Figure 6.3.0.1: Model Tracking Testbed Data Flow 
When drift is detected, a recalibration is activated which re-estimates the $\mathrm{H}_{\mathrm{k}}$ matrix, and the noise parameters of the new data in the sequence, after which it returns to tracking.

\subsubsection{Testbed Components}

Five main components are needed:

- LSTM model to learn initial $\mathrm{H}_{\mathrm{k}}$ matrix parameters

- $\quad$ LSTM model to learn Q, R \& F matrix parameters

- Kalman filter to track model parameters

- LSTM model to relearn drift $\mathrm{H}_{\mathrm{k}}$ matrix parameters

- Model Training \& Tracking Data Generators (with noise)

Lastly a control script is needed to execute the life cycle of the components. The 'learning threshold' used to determine when drift has occurred in the underlying $p$ vector and trigger recalibration of the $\mathrm{H}_{\mathrm{k}}$ matrix is configured by the user. A suitable threshold is chosen by observing test data.

An alternative approach suggested for determining the 'learning threshold' is to do several runs with known $p$ vector values. These runs would have known changes in the $p$ value, and the response of the Kalman filter tracker via an increase in prediction error is logged. The standard deviation (or a suitable statistical error) in the error change can be used to determine a suitable threshold, providing an automated method for threshold determination.

An analysis of the tracking testbed will be covered in the following chapters. 


\subsection{2 $\mathrm{H}_{\mathrm{k}}$ Matrix Prediction LSTM Model}

This component's logical flow is shown in Figure 6.3.0.1. The basic structure of the learning unit is the same as the $\mathrm{Q} \& \mathrm{R}$ matrix learning unit structure. The dimensions of the neural network are different $(|p|)$, and so are the cost function and optimization method.

The goal of the LSTM model is to estimate the unknown model parameter vector $p$ given measurable model parameters. The input and output layers of the LSTM network must therefore match the size of the measured and unknown parameter vectors.

The cost function is a simple mean square error on the output, while the optimizer is a momentum gradient descent on the cost function.

\subsubsection{Model Output \& Training Error}

In order to create a tensor [109] to calculate the error on the model output $p$, it is necessary to evaluate the LQN model parameters associated with the $p$ vector, from within the GPU. Neural network models using tools like Tensorflow [109] are designed to run on the GPU for speed and scale purposes. Only operations expressed as tensor operations can run on the GPU. Hence, since it is not possible to run the LQN system solver in the GPU environment, it was necessary to create a lookup table tensor operation representing the search space of LQN model parameters relevant to the system under consideration. This tensor is the error function [1]:

$$
c o s t=\sum_{n=1}^{N_{B A T C H}} l q n t b l\left[\operatorname{row}\left(p^{n}\right)\right]-y^{L Q N n}
$$


where lqntbl is the labelled LQN model table, $y^{L Q N n}$ is the measured parameters vector, $\mathrm{p}_{\mathrm{n}}$ is the unknown parameters vector and $\operatorname{row}\left(p^{n}\right)$ [1] is a function that computes the LQN solver output table row from a parameter vector by place value summation [1]. Place values are specified in the config YAML file as the 'lqn-range' configuration.

Overall the performance model tracking testbed provides an algorithm displaying quick convergence to model parameters $p$ from measurement data, and the ability to detect changes in measurement data requiring recalculation of $p$. An analysis of the tracking testbed will be covered in the system analysis chapter. 


\section{Chapter 7: Experiment Plan}

Now that the system architecture has been covered in the previous chapter, it is necessary to present an evaluation of it. This chapter covers the experiment design influencing the experiments and testing in this project. It provides general information about the experimental setup and can serve as a guideline for the details of any experiment encountered in the project. Section 7.1 describes the workload principles and characterization. Section 7.4 covers experiment tuning involving arrival rates, and section 7.5 provides the details of the key characteristics of each experiment, and the outline of the experiment plan's design.

The overall goal of this research is to demonstrate effective use of machine learning with Kalman filter tracking of an LQN model, and to show that KF-PCA is an effective method. The key components of the experimental plan with respect to this goal include:

1. Verify resource usage data during tuning and operational modes show reasonable operation of the control system, and highlight any skews or divergence

2. Verify that the KF-LSTM (section 6.1.3.3) training process is working correctly, with reduction in error and/or increase in accuracy over a large number of training epochs

3. Verify that the LSTM does improves KF accuracy in KF-LSTM algorithm.

4. Evaluate KF-LSTM model tracking behaviour over a step change in model parameter values with random noise 
5. Evaluate KF-PCA model tracking behaviour and compare it to KF-LSTM model tracking over a step change in model parameter values with random noise

6. Show that system control is effective over step changes in model parameters and varying workloads

Various approaches are taken to provide a robust experimental design encompassing aspects of performance analysis concepts [82], measurement techniques and tools involving workload selection and characterization, software and distributed system monitors, capacity planning, data presentation principles, and factorial experimental design.

\subsection{Workload Selection \& Characterization}

This section describes the workload selection and characterizes its nature and level of detail relevant to experiments in this project. Much like the SPEC benchmark suite ([82] Section 4.6.8), and the SPEC Cloud IaaS 2018 benchmark [83], a workload suitable for benchmarking cloud platforms is selected and characterized. Four aspects of the workload are considered: its services, level of detail, representativeness and timeliness [107].

The workload's services are the client requests from external sources. The level of detail provided involves client request arrival rates, response times, and request types. The test clients generate http GET requests with arrival rate $1 \mathrm{~ms}$ (1000 requests per second).

Typical response times are less than $300 \mathrm{~ms}$ as observed by the test clients in this project. Any response times less than $1 \mathrm{~ms}$ are usually considered instantaneous and are thus ideal, 
with any response times over 1s interrupting the users flow of thought, and any above 10s will lose the user's attention [107] . The generated client requests have selected rates per type, and expected response times. Subsystem components under test (CUT) are monitored to track operating system utilization levels, CPU utilizations, memory usage and process metrics. Trace information via logging is used to track the arrival rates and demands for each CUT. These are stored in pickle files generated after each test case. Representativeness in terms of request arrival rates, demands and resource usage for the SUTs is ensured such that these closely match expected values for real systems. In order to ensure timeliness of the workload, real CUTs such as database and search engine components can be represented by test applications simulating their behaviour while providing reasonable response times $(<500 \mathrm{~ms})$ for request processing, provisioning and deprovisioning actions.

Workload characterization techniques capture system views of workload parameters and features. Averaging, dispersion, histograms, Markov models \& clustering are typical techniques. The request arrival rates are fixed in value, but vary across test scenarios. The following section covers the details of request arrival rate distributions, request types and associated SUT configurations.

\subsection{Tuning Experiments}

For each scenario, various testing steps are followed to characterize the behaviour of the system under different load regimes and configuration environments. The principles of 
blackbox testing are done where the test cases are agnostic towards the factor combination setup. These are the phases followed in each test scenario:

1. Persistence targeting request traffic (R1) of specified arrival rates (see arrival rate test details section below)

2. In-memory search request traffic (R2) of specified arrival rates

3. Application cache request traffic (R3) of specified arrival rate

4. Mixed random request traffic (R4) of varying arrival rates

\subsubsection{Arrival Rates}

Arrival rates are referred to using the symbol $\lambda$. For each scenario, client requests to the SUT are set up to gradually increase from upper bound $(\chi=1 / 1 \mathrm{~ms}=1000)$ to lower bound $(\chi=1 / 10 \mathrm{~s}=0.1)$ depending on the application under test, to gradually approach system saturation. Saturation is defined here as the condition where system response time diverges or system failure occurs, or when usability is adversely affected (see section 7.1). The request types examined in each test scenario (as described in the main section above) are shown below:

1. Resource usage data goal: 10 s of R1, then 10 s of $\mathrm{R} 2$, then 10 s of $\mathrm{R} 3$

2. KF-LSTM training process goal: 10s of R1, then 10s of R2, then 10s of R3

3. LSTM improves KF accuracy goal: 10s of R1, then 10s of R2, then 10s of R3.

4. KF-LSTM model tracking behaviour: 10s of R1, then 10s of R2, then 10s of R3 
5. Evaluate KF-PCA model tracking behaviour and compare it to KF-LSTM model tracking over a step change in model parameter values with random noise goal: 10s of R1, then 10 s of R2, then 10 s of R3

6. Show that system control is effective over step changes in model parameters and varying workloads: 10 s of $\mathrm{R} 1$, then 10 s of $\mathrm{R} 2$, then 10 s of $\mathrm{R} 3$, and 10 s of $\mathrm{R} 4$

\subsection{Experimental Factors}

Factors considered in the design of test scenarios include the overall controller, LSTM unit, Kalman Filter (KF) unit, and fixed LQN model \& generative model. An evaluation of each factor is done with the goal of determining its effect and variation on overall performance. Various performance aspects are considered.

A factored model with replication describes the experimental approach (chapter 18 in [82]). Replication errors and confidence intervals are computed for relevance determination of results.

Scenarios will include testing with and without Kalman Filter tuning (with/without LSTM), basic and predictive control (section 6.1.4.1), multiple provisioning burst sizes, multiple virtual machine types, and all done statistically over multiple iterations. Summaries of these test scenarios are provided in the system evaluation chapter. 
Table 7.3.0.1: Component Evaluation Test Scenarios

\begin{tabular}{|c|c|c|c|}
\hline Section & Tracking Algorithm & Measure & Goal \\
\hline 8.2 .1 & KF-LSTM1 & Mean Squared Error & $\begin{array}{l}\text { Training success/failure; } \\
\text { compare to KF-LSTM2 }\end{array}$ \\
\hline 8.2 .2 & KF-LSTM2 & Accuracy & $\begin{array}{l}\text { Training success/failure; } \\
\text { compare to KF-LSTM1 }\end{array}$ \\
\hline 8.3 & KF vs KF-LSTM1 & KF Accuracy & Compare KF/KF-LSTM \\
\hline 8.4 .2 & KF-LSTM1 & Variance & $\begin{array}{l}\text { Convergence of KF } \\
\text { coefficient matrices }\end{array}$ \\
\hline 8.5 & KF-LSTM1 & Parameter Value & Show model tracking \\
\hline 8.6 & KF-PCA & Parameter Value & Show model tracking \\
\hline 8.6 .1 & $\begin{array}{l}\text { KF-PCA vs } \\
\text { KF-LSTM1 }\end{array}$ & $\begin{array}{l}\text { Mean Error \& } \\
\text { Variance }\end{array}$ & Compare model tracking \\
\hline 8.7 & Control System & Mean \& Variance & $\begin{array}{l}\text { Compare active to passive } \\
\text { control }\end{array}$ \\
\hline
\end{tabular}


Table 7.3.0.2: Controller Evaluation Test Scenarios

\begin{tabular}{|l|l|l|l|l|l|}
\hline Section & $\begin{array}{l}\text { Tracking } \\
\text { Algorithm }\end{array}$ & Control & BR & Metric & Goal \\
\hline 9.1 & KF-PCA & Predictive & $3,7,11$ & $\begin{array}{l}\text { CPU, } \\
\text { Shared \& \% } \\
\text { Memory } \\
\text { usage }\end{array}$ & $\begin{array}{l}\text { lompare } \\
\text { step } \\
\text { response }\end{array}$ \\
\hline 9.2 & KF-PCA & Predictive & $3,7,11$ & $\begin{array}{l}\text { CPU, } \\
\text { Shared \& \% } \\
\text { Memory } \\
\text { usage }\end{array}$ & $\begin{array}{l}\text { Statistical } \\
\text { comparison }\end{array}$ \\
\hline 9.3 & KF-PCA & Non-predicti & $3,7,11$ & $\begin{array}{l}\text { CPU, } \\
\text { Shared \& \% } \\
\text { Memory } \\
\text { usage }\end{array}$ & $\begin{array}{l}\text { Statistical } \\
\text { comparison }\end{array}$ \\
\hline 9.4 & KF-PCA & Predictive & 1 & $\begin{array}{l}\text { CPU, } \\
\text { Shared \& \% } \\
\text { Memory } \\
\text { usage }\end{array}$ & $\begin{array}{l}\text { Statistical } \\
\text { comparison } \\
\text { by VM type }\end{array}$ \\
\hline 9.4 .1 & KF-PCA & Predictive & $1,3,10,20$ & $\begin{array}{l}\text { CPU, } \\
\text { Shared \& \% } \\
\text { Memory } \\
\text { usage }\end{array}$ & $\begin{array}{l}\text { Statistical } \\
\text { comparison } \\
\text { by VM type }\end{array}$ \\
\hline
\end{tabular}




\section{Chapter 8: Analysis}

This chapter examines the effectiveness of the control system components. The purpose is to fulfil the research goals of evaluating the impact of machine learning on tuning Kalman filters, and also of evaluating KF-PCA as an algorithm for estimating model parameters. Overall, machine learning improves the Kalman Filter prediction accuracy (see below), with a high level of fluctuation in performance over the first training epochs which is as expected [6], which stabilizes as the number of epochs rises with Kalman Filter accuracies consistently above $90 \%$ for higher epochs. More detailed discussion of this is in the epoch impact analysis in the following subsections.

One key part of evaluation is the Kalman Filter accuracy, defined as:

$K F$ Accuracy $=\max \left(0,1-\frac{\left|\widehat{x}(i)_{k \mid k-1}-\widehat{z}(i)_{k}\right|}{N(i)}\right)$,

where $\mathrm{N}(\mathrm{i})$ is the normalization factor for metric i (equal to its maximum absolute value in the data record being evaluated - see Section 6.1.3.1), $\widehat{x}(\mathrm{i})_{\mathrm{kk} k-1}$ is the predicted value at time step $\mathrm{k}$, and $\mathrm{z}(\mathrm{i})_{\mathrm{k}}$ is the measured value for time step $\mathrm{k}$.

\subsection{Resource Usage Data Parsing \& Normalization}

In order to fulfil the research goal of using LSTM to tune KF, it is important to parse and sanitize input data. This is measurement data from application system processes. The 
behaviour of these processes (described in Section 6.1.3.1 - sorted by process identifier PID) is shown in the surface plot below. This type of data is used in the evaluations in subsequent sections. From the colour legend, blue indicates low resource utilization, while red indicates high resource utilization, normalized as per section 6.1.3.1. Note that the plot has a scale $[-1,1]$, a feature of the display library, not the data.

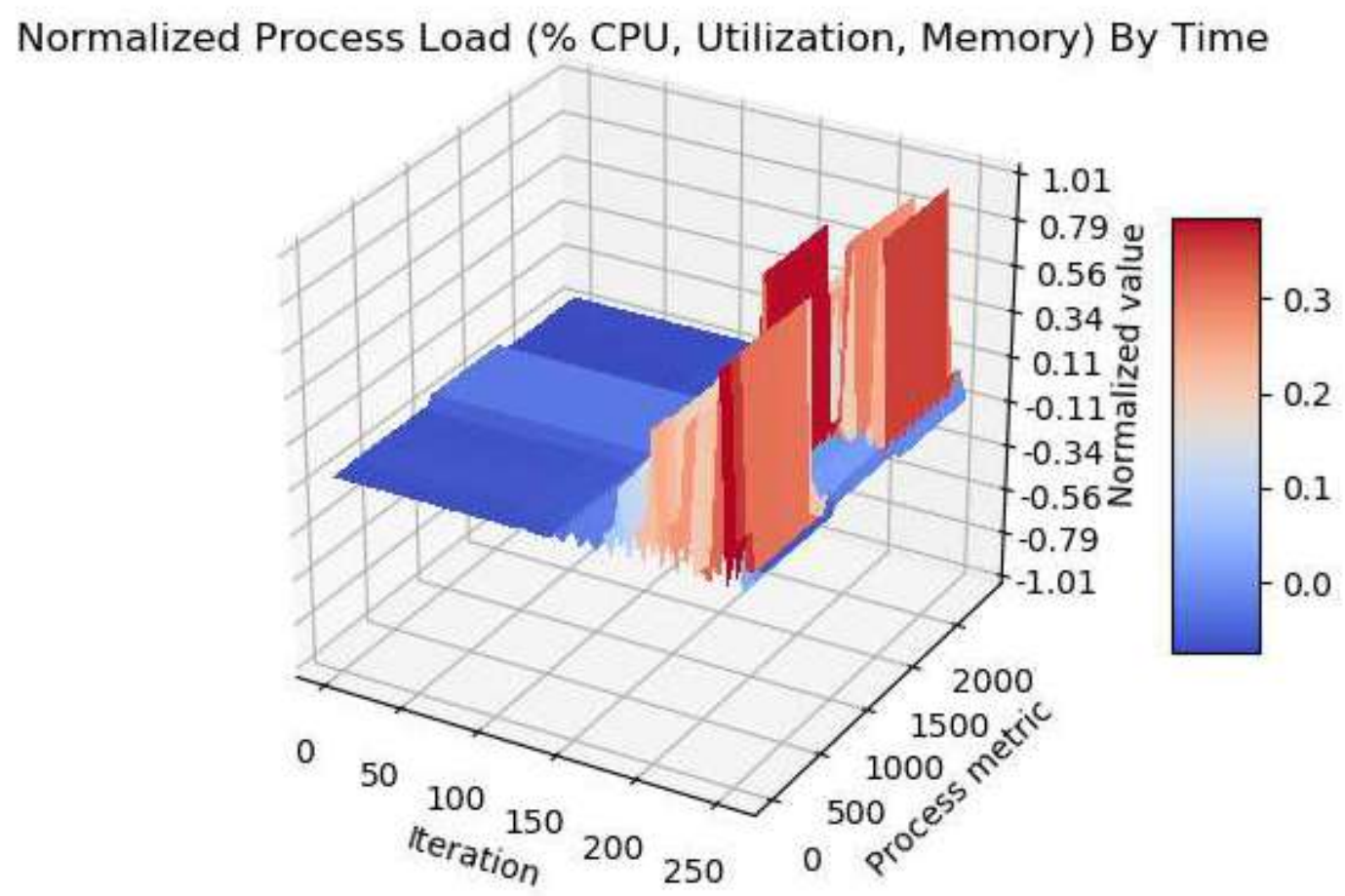

Figure 8.1: Normalized Process Resource Utilization Over Time

The high resource usage shown towards the end is likely due to heavier processing in the final epochs of the LSTM which was able to impact most processes on the system, on most metrics. This kind of dynamic input is richer for training the LSTM unit and leads to better trained LSTM models, which in turn leads to more robust outputs. Measurements are parsed into numbers and normalized to the $[0,1]$ range (figure 8.1) 


\subsection{Tuning Of The LSTM}

This section describes the process of training the KF-LSTM algorithm (Section 6.1.2.3), fulfilling the research goal of evaluating the tuning of KF using LSTM neural networks. Selection of the variant to use is dependent on the speed of convergence during training based on number of epochs. Overall the KF-LSTM1 variant is selected after evaluation since both variants show reasonable improvement by epoch during LSTM training, but KF-LSTM1 does not rely on ordinal class categorization (but uses regression instead) avoiding the need to select classes for values close to arbitrary class boundaries, and the potential limits of a finite class range.

\subsubsection{Tuning of the Regression LSTM Unit (KF-LSTM1)}

This section covers the linear regression variant of KF-LSTM. The training cost function is defined as the mean square difference between the model parameters and label data. This is calculated using Tensorflow during the training phase.

In the testing phase, the mean squared error is as defined in section 6.1.2.3.1. A plot of error by epoch is shown below: 


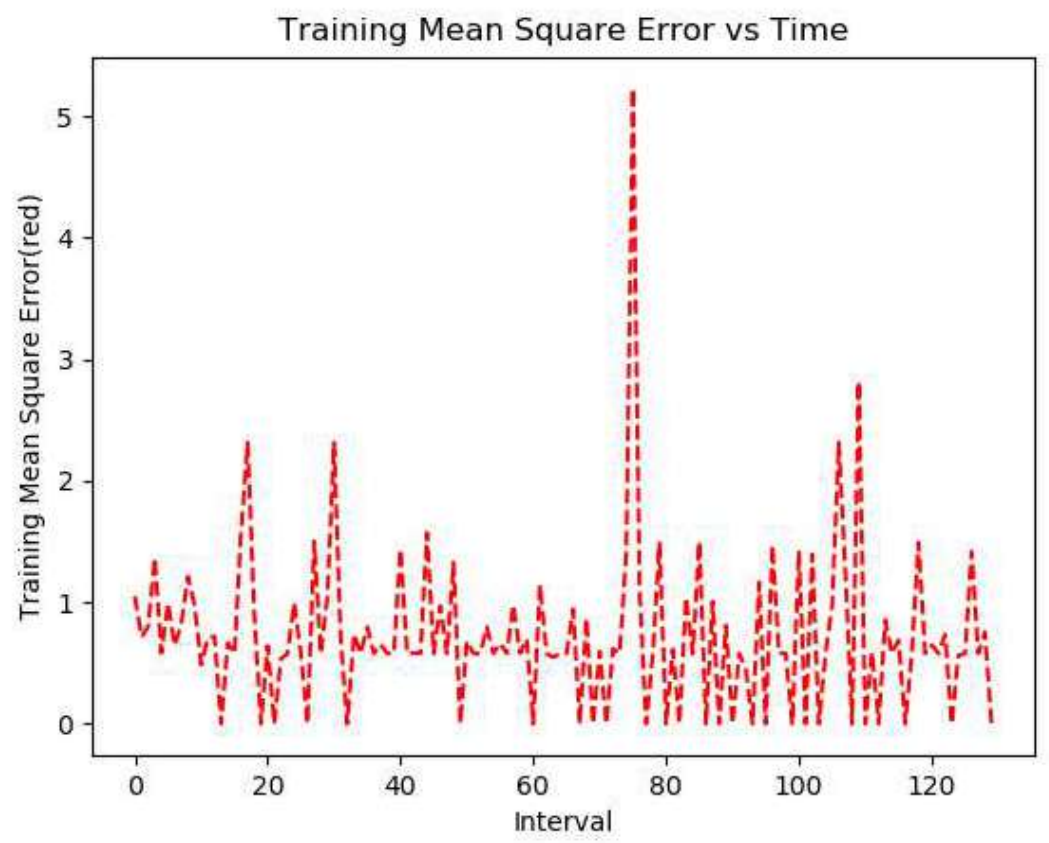

Figure 8.2.1: Reducing MSE (Mean Square Error) over multiple training epochs The cumulative MSE reduces rapidly and stabilizes at around 70 intervals.

\subsubsection{Tuning of Classification LSTM (KF-LSTM2)}

This section defines the classification accuracy of the LSTM unit This section covers the linear regression variant of KF-LSTM. The training cost function is defined as the mean square difference between the model parameters and label data. This is calculated using Tensorflow [109] during the training phase.

In the testing phase, the classification accuracy is as defined in section 6.1.2.3.2. A plot of the accuracy by epoch is shown below: 


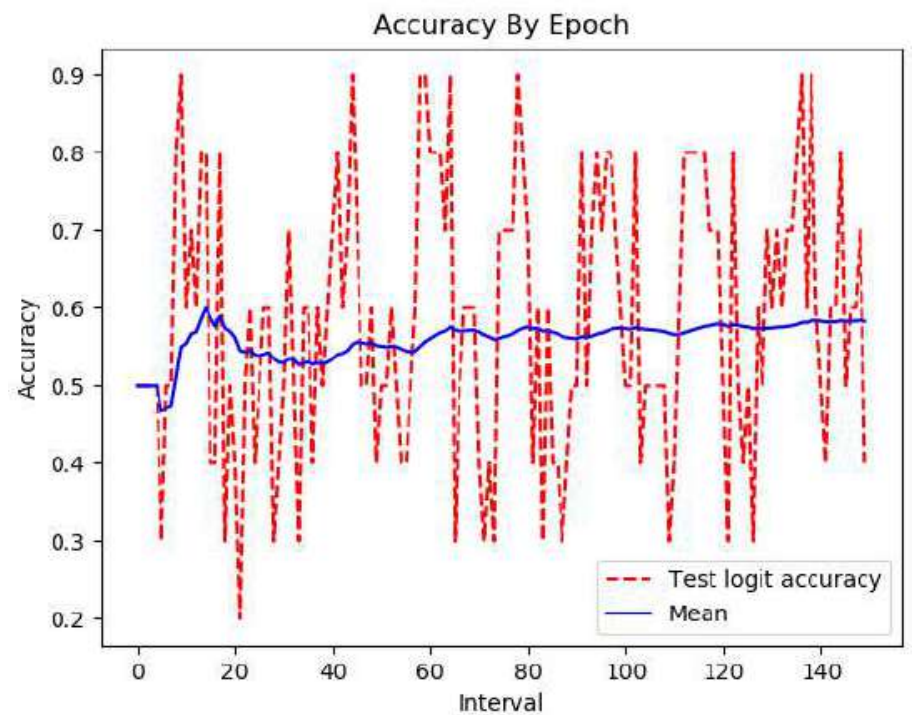

Figure 8.2.2: Logistic classification accuracy by epoch (increasing accuracy)

Classification accuracy increases slowly and converges at around 120 intervals.

The KF-LSTM1 variant is selected since both variants show reasonable improvement by epoch during LSTM training, but KF-LSTM1 needs fewer epochs to converge (70 versus 120).

\subsection{KF-LSTM1 Impact on Kalman Filter Prediction Accuracy}

The value of using machine learning to tune the KF is examined by comparing KF-LSTM1 with an untuned KF with fixed Q and R parameters, in tracking and predicting values for a set of deterministic and random time-sequences.

This section examines the KF-LSTM1 algorithm in control mode, using measurement data generated from the control unit to evaluate KF-LSTM1 prediction accuracy. It compares and contrasts the basic KF and KF-LSTM1 algorithms. As seen in the previous 
section, KF-LSTM1 after a long period shows a reduction in mean square error with training, hence machine learning is effective for improving the basic KF.

This section attempts to identify and quantify differences in performance in those two scenarios.

Notation: $\mu(m, s)$ represents a normal distribution with mean $\mathrm{m}$ and standard deviation $\mathrm{s}$ The cases considered were:

1. A deterministic time function $\mathrm{z}_{\mathrm{k}}=1.01^{\mathrm{k}}$

2. A deterministic time function $\mathrm{z}_{\mathrm{k}}=\exp (\mathrm{k}) \bmod 50$

3. A normally distributed time function with independent samples $z_{k}=\mu(0,1)$

4. A sine wave with normally distributed errors $\mathrm{z}_{\mathrm{k}}=\sin (k)+0.3 \mu(0,1)$

5. A square waver $z_{k}=0.3 \mu(0,1)$

6. A repeated square wave $z_{k}=\left\{\begin{array}{lll}1 & \text { if }\lfloor x / 50\rfloor & \bmod 2=0 \\ 0 & \text { if }\lfloor x / 50\rfloor & \bmod 2 \neq 0\end{array}\right.$

N.B: The untuned KF has Q \& R noise matrices set to null matrices, and F matrix set to identity. The tuned KF has these matrices set by the LSTM unit as in section 8.4.1. The approach taken is to track a known signal using a Kalman filter. The signal will typically contain noise. A plot is then presented of the raw signal, the predictions made by the raw (untuned) filter, and also those of the tuned filter. A separate plot is also presented showing the raw signal, and the prediction errors of both the raw and tuned filters. Where needed, the plot may be zoomed in to a subrange to highlight interesting behaviour. Also, summary statistics are shown presenting the mean and standard 
deviation of the error values to capture any quantitative differences between the two filter types.

For an analysis of coefficient convergence during tuning, please see Section 8.4 .2 below. The plot below shows the trajectories of prediction accuracies for plain vanilla (red) and LSTM tuned (green) Kalman filters. Their accuracies were evaluated for a duration after tuning where measurement samples were compared to their a priori state predictions. Note that both Kalman filters are initially tuned for a time using real time system measurements, and are updated accordingly both during this phase and also after each prediction.
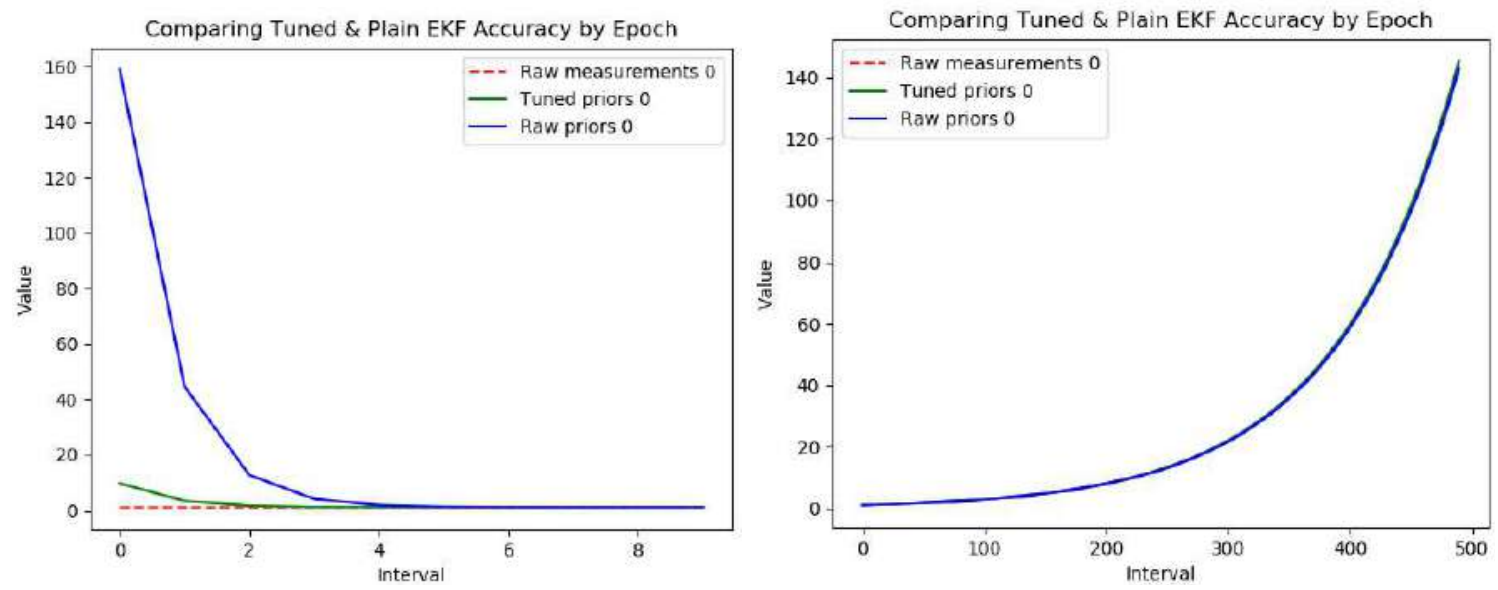

Figure 8.3.0.0: EKF predictions for $\mathrm{z}_{\mathrm{k}}=1.01^{\mathrm{k}}$ at different scales 


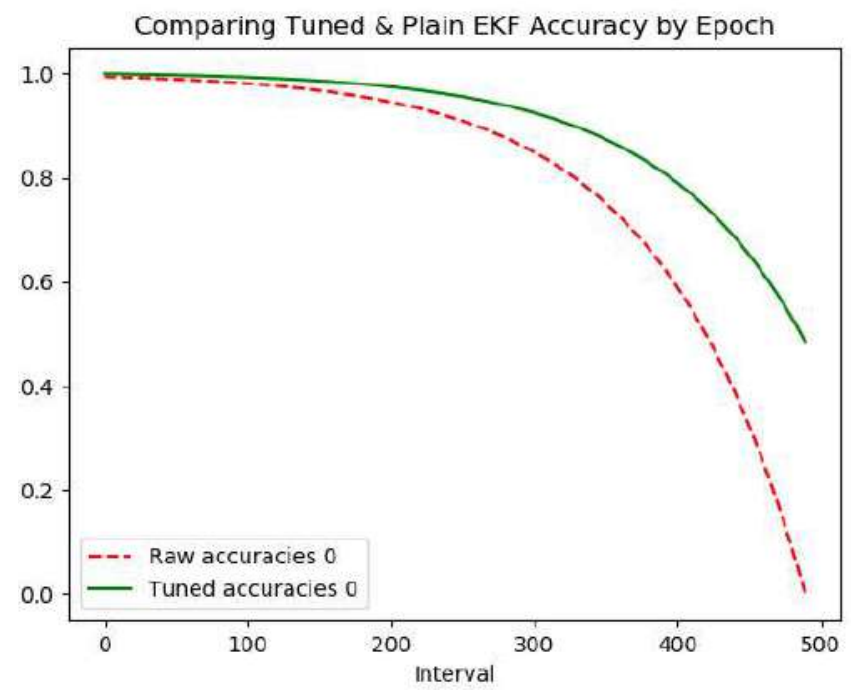

Figure 8.3.0.1: EKF prediction accuracy for $\mathrm{z}_{\mathrm{k}}=1.01^{\mathrm{k}}$

Tuned accuracy: std $=0.12857$, mean $=0.89415$

Raw EKF accuracy: std $=0.48472$, mean $=0.600928$

NB: Faster convergence and higher accuracies for the tuned EKF

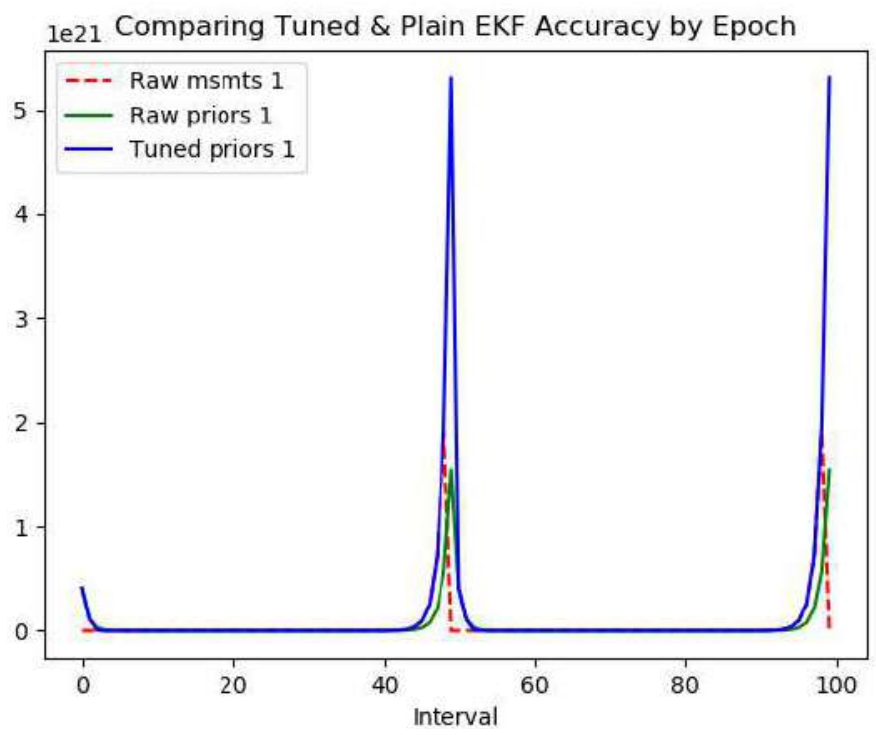

Figure 8.3.0.4: Raw \& Tuned EKF predictions for $\mathrm{z}_{\mathrm{k}}=\exp (\mathrm{k}) \bmod 50$ (100 time steps) 
NB: For the exponential function, the accuracies for the tuned EKF spike higher showing better prediction of the function divergence

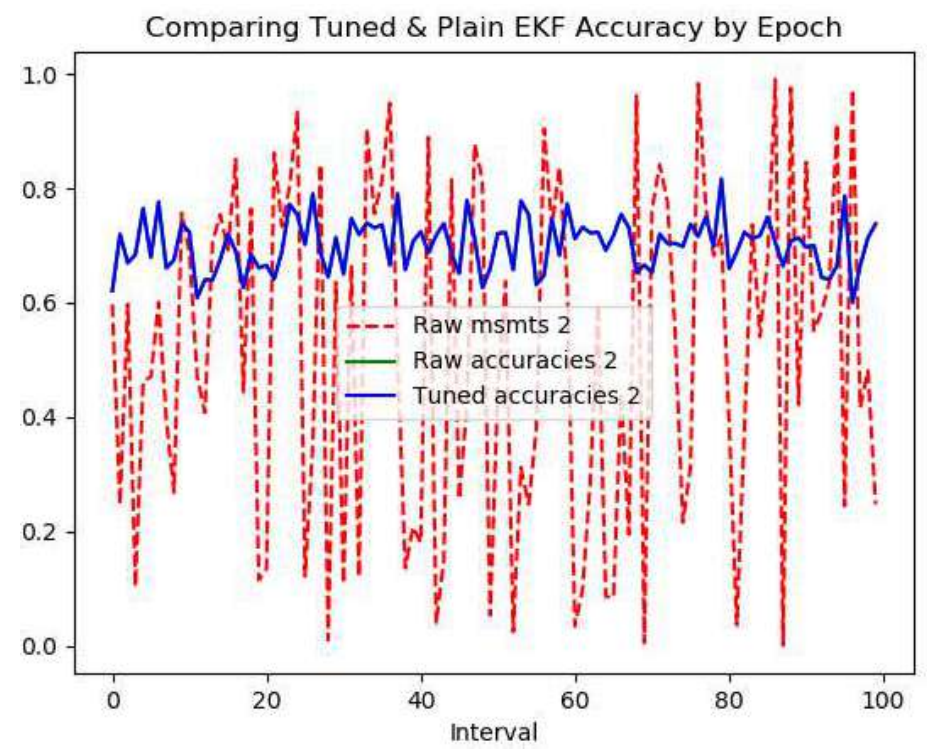

Figure 8.3.0.5: Raw \& Tuned EKF accuracies for $\mathrm{z}_{\mathrm{k}}=\mu(0,1)(19)$

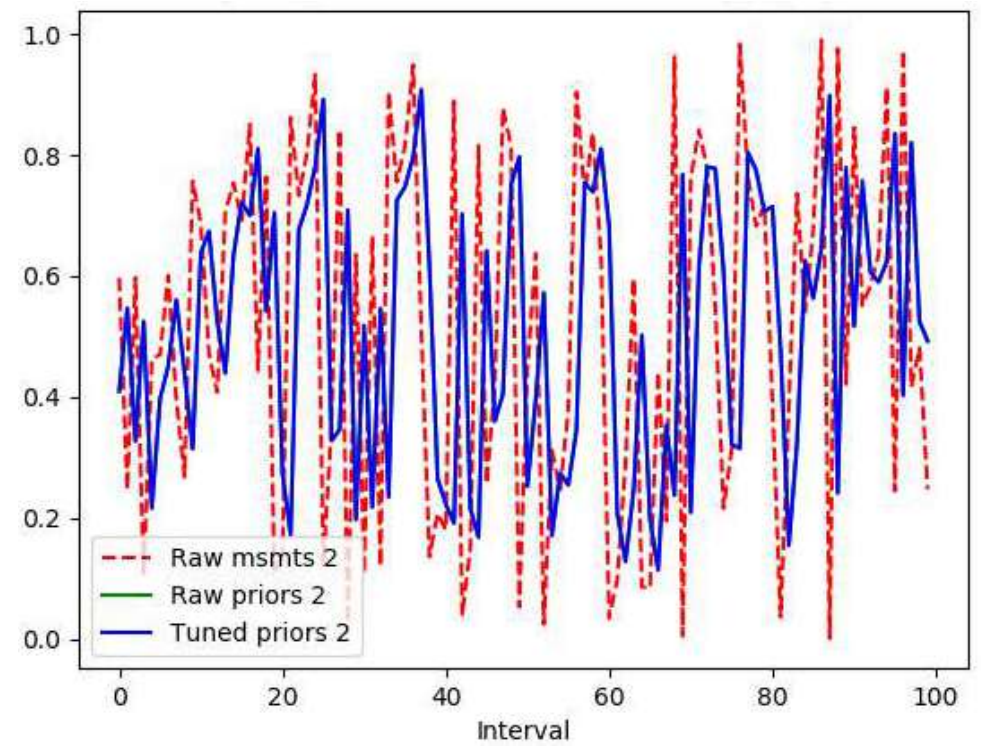

Figure 8.3.0.6: Raw \& Tuned EKF predictions for $\mathrm{z}_{\mathrm{k}}=\mu(0,1)$ (100 time steps) 
NB: For the random normal signal, there's little gain with tuning with identical predictions for both filters

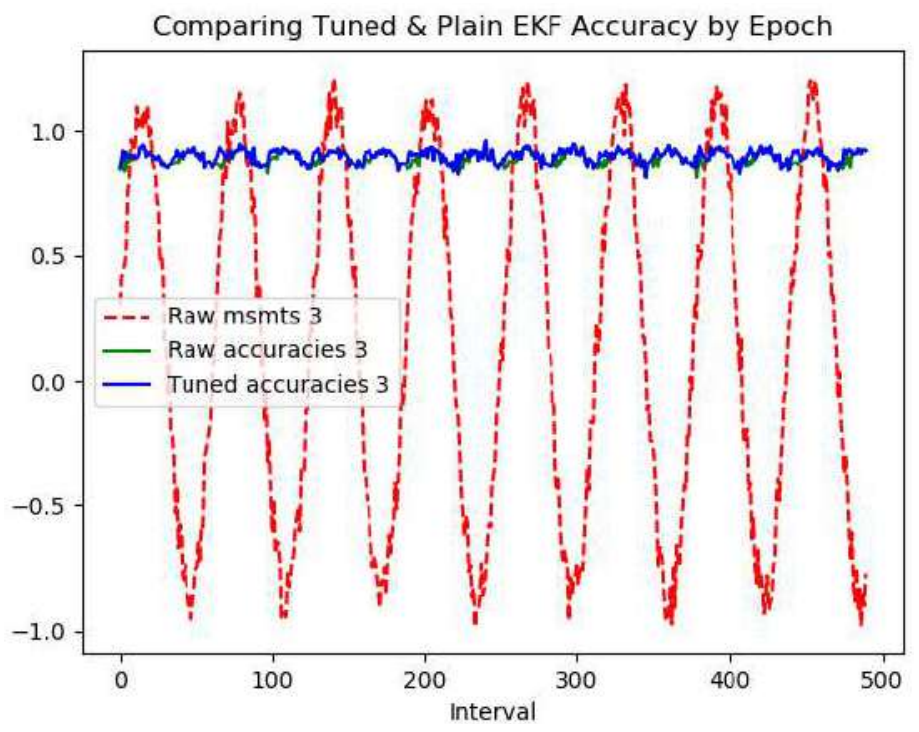

Figure 8.3.0.7: EKF Raw \& Tuned accuracies for $\mathrm{z}_{\mathrm{k}}=\sin (k)+0.3 \mu(0,1)$

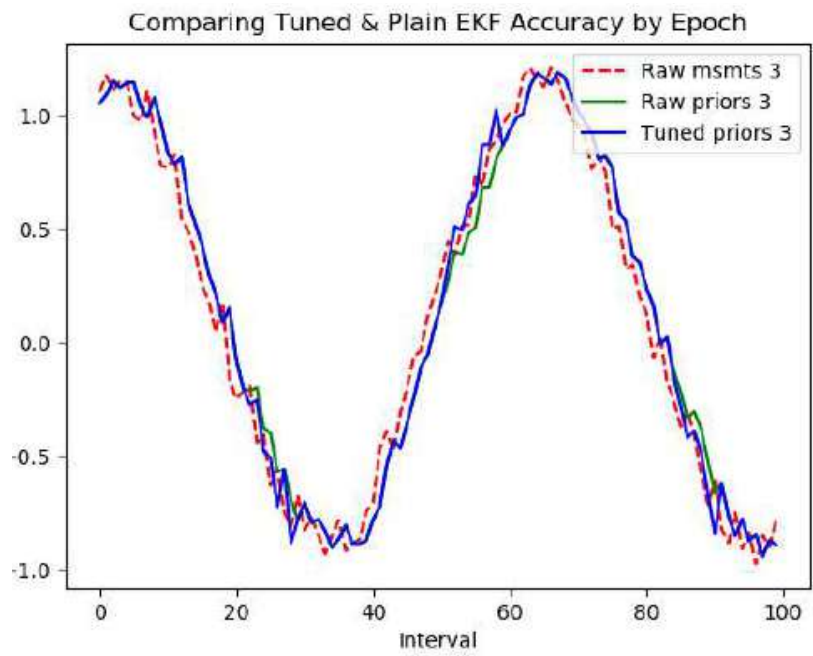

Figure 8.3.0.8: Predicted priors for raw and tuned EKFs tracking $\mathrm{z}_{\mathrm{k}}=\sin (k)+0.3 \mu(0,1)$

Tuned: std $=0.02570$, mean $=0.89977, \max =0.96192$

Raw: std $=0.02784$, mean $=0.89191, \max =0.96192$ 
NB: For the noisy sine wave, the tuned Kalman filter has higher mean accuracy

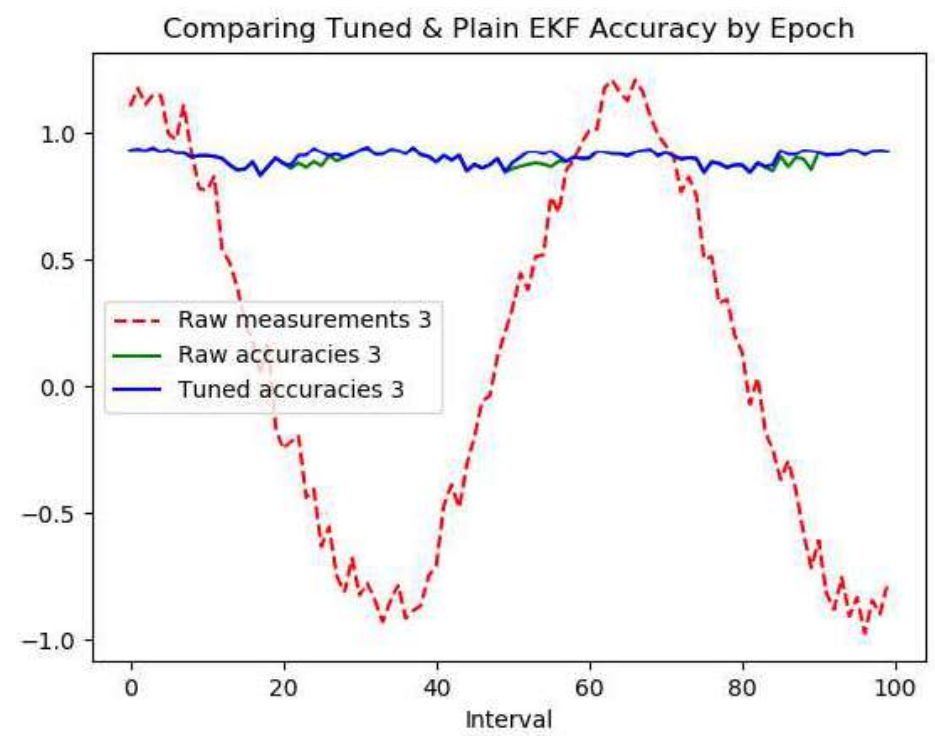

Figure 8.3.0.9: Raw \& Tuned EKF accuracies for $\mathrm{z}_{\mathrm{k}}=0.3 \mu(0,1)(100$ time steps $)$

$$
z_{k}=\left\{\begin{array}{lll}
1 & \text { if }\lfloor x / 50\rfloor & \bmod 2=0 \\
0 & \text { if }\lfloor x / 50\rfloor & \bmod 2 \neq 0
\end{array}\right.
$$

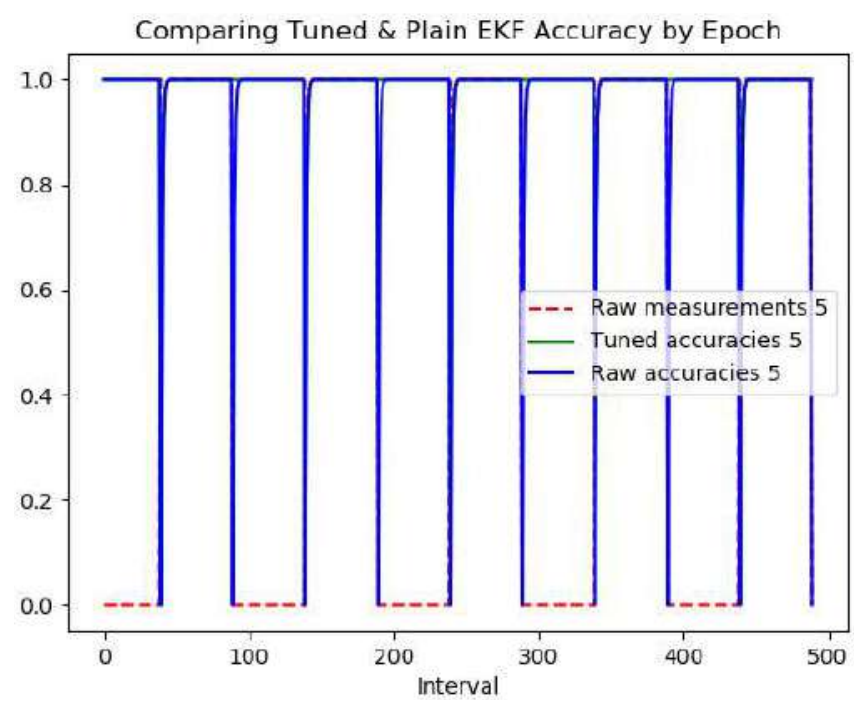

Figure 8.3.0.10: Raw \& Tuned EKF accuracy for square wave (eq (21))

Tuned accuracies: $\mathrm{std}=0.103919$, mean $=0.986061, \max =1.0$ 
Raw accuracies: std $=0.145234$, mean $=0.972869, \max =1.0$

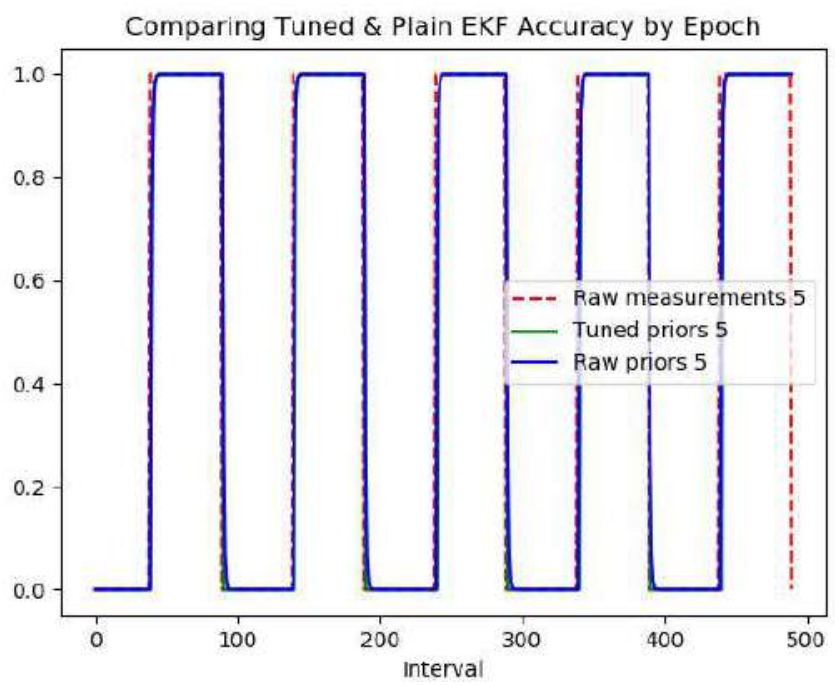

Figure 8.3.0.11: Raw \& Tuned EKF predictions for eq (21)

NB: The Tuned Kalman filter performs better in step function tracking as well, with higher mean accuracy (section 8.3) and lower standard deviation.

In a case like the noise signal $\left(\mathrm{z}_{\mathrm{k}}=\mu(0,1)\right.$ - figure 8.3.0.6), a purely random signal, both tuned and raw KF are equally inaccurate since there is no information for the tuned KF to learn. In general, the tuned KF had a higher mean accuracy (and a lower standard deviation). The best case was the exponential $\mathrm{z}_{\mathrm{k}}=1.01^{\mathrm{k}}$ with higher accuracy (tuned mean $=0.8942$, raw mean $=0.6009$ - figure 8.3.0.1), and also the sinusoid $\mathrm{z}_{\mathrm{k}}=$ $\sin (k)+0.3 \mu(0,1)($ tuned mean $=0.8998$, raw mean $=0.8919-$ figure 8.3.0.7). Other test cases also had higher mean accuracy after tuning like the step signal $z_{k}=\left\{\begin{array}{lll}1 & \text { if }\lfloor x / 50\rfloor & \bmod 2=0 \\ 0 & \text { if }\lfloor x / 50\rfloor & \bmod 2 \neq 0\end{array}\right.$ (tuned mean $=0.9861$, raw mean $=0.9729-$ figure 8.3.0.10) . 


\subsection{KF-LSTM1 Learning of KF Parameters}

This section is about the tuning of KF parameter matrices by the LSTM unit, considering their accuracy and speed of convergence during tuning mode.

\subsubsection{Estimation of EKF Q, R \& F Matrix Coefficients}

This section examines the KF-LSTM1 algorithm in the tuning phase, using measurement data generated from the control unit. The noise and state transition matrices $(\mathrm{Q}, \mathrm{R}$ and $\mathrm{F}$ matrices respectively) are produced by the LSTM prediction layer either as diagonal matrices (with zeros for upper and lower half submatrices), and as full matrices. The diagonal matrix approach had higher speed of convergence and KF accuracy after tuning. The table below is for an application system whose state model is equivalent to $\mathrm{F}=\mathrm{I}$, the identity matrix. The KF is going through tuning, and learns this model as shown in the table below.

N.B: In the table below, columns represent 4 sample diagonal entries of $\mathrm{F}$ (which has 24 diagonal entries in total):

Table 8.4.1.1: Discovered F-diagonal coefficients for real and simulated systems

\begin{tabular}{|l|l|l|l|l|}
\hline $\mathrm{F}[0,0]$ & $\mathrm{F}[1,1]$ & $\mathrm{F}[2,2]$ & $\mathrm{F}[3,3]$ & LSTM output (KF F matrix - note above) \\
\hline 4.8306 & 2.4785 & 0.9600 & 1.9170 & $\mathrm{k}=100\left(\mathrm{z}_{\mathrm{k}}=0\right)$ \\
\hline 1.0 & 1.0 & 1.0 & 1.0 & $\mathrm{k}=900\left(\mathrm{z}_{\mathrm{k}}=0\right)$ \\
\hline 1.01 & 1.01 & 1.01 & 1.01 & $\mathrm{k}=500\left(\mathrm{z}_{\mathrm{k}}=1.01^{\mathrm{k}}\right)$ \\
\hline $1 \mathrm{e}-9$ & $1 \mathrm{e}-9$ & $1 \mathrm{e}-9$ & $1 \mathrm{e}-9$ & $\mathrm{k}=500\left(\mathrm{z}_{\mathrm{k}}=\mathrm{eq}(21)\right)$ \\
\hline
\end{tabular}


The samples above reflect the discovery of the state transition matrix $(\mathrm{F})$ diagonal.

The F matrix coefficients are driven by the activity on the measured system, varying depending on which application process loads the system most heavily, indicating the rates of increase of each model parameter. Convergence for F matrix entries is slower than for the Q \& R noise coefficients, determined primarily by the feature selection for this matrix. Convergence follows the pattern of the noise matrices, and is covered in detail in the next section below.

\subsubsection{Convergence of EKF Coefficients In Tuning Mode}

This section examines the KF-LSTM1 algorithm in the tuning phase, focussing on the convergence of the tuned input parameters of the KF using measurement data from the control unit. The figures below show the coefficient values used during tuning. On the left is an aggregate surface plot of all normalized coefficients, while that on the right is of a single one. The initially fluctuating values produced by the LSTM unit quickly stabilize at around 100 iterations in, and fully converge by 800 iterations.

The coefficient gradient converges to near zero quickly as well, with a mean $(\mu)$ and standard deviation $(\sigma)$. The first 100 iterations of the actual coefficient have $\mu=4.589, \sigma=3.178$, and the rest are closer to $\mu=0.5344, \sigma=9.544 e-4$.

With the following definitions:

- Tolerance $\varepsilon$, at a confidence of $95 \%$, and 
- $\sigma_{w}$ the standard deviation of a set of consecutive 100 iteration windows (see figure below).

Convergence is then defined as $\sigma_{w} \leq \varepsilon, \varepsilon=0.03$

The confidence is calculated over each iteration window and tuning stops once the confidence is above the chosen threshold. The coefficients converge at a rate of around 800 iterations.

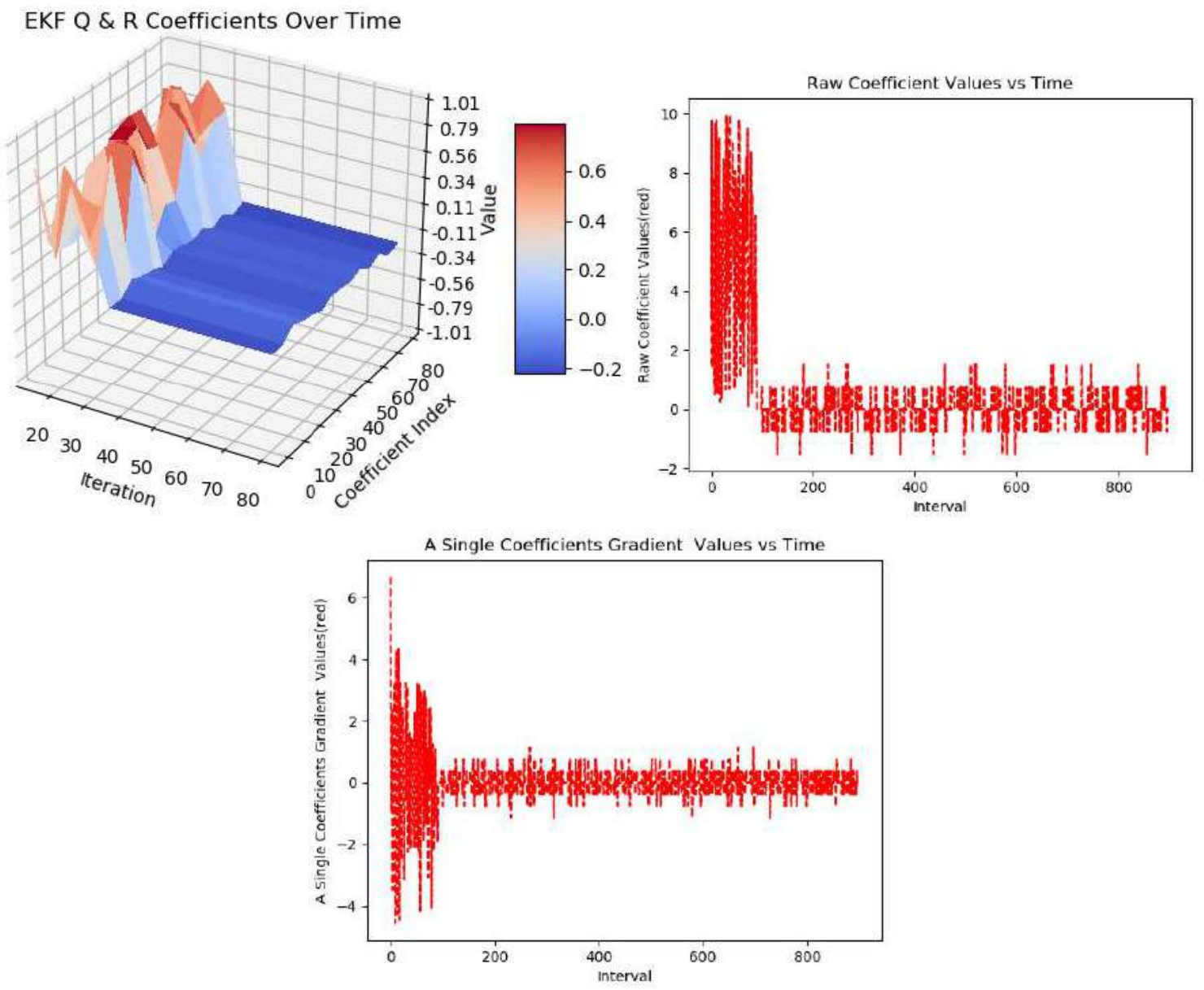

Figure 8.4: Aggregate coefficients, individual coefficients \& gradients by iteration NB: Absolute values of the coefficients are used as noise coefficients in the Q \& R matrices. 
The KF Q \& R coefficients converge at a rate of around 800 iterations, using a standard deviation tolerance of 0.03 with a confidence of $95 \%$.

\subsection{KF-LSTM1-Based Model Tracking Testbed Analysis}

This section examines the KF-LSTM algorithm in control mode, adopted to use test measurement data generated from the control unit to track the system model. The purpose is to evaluate direct KF-LSTM-based of model tracking and ensure it works. In conclusion, the KF-LSTM1 model tracking estimator was effective at detecting changes in and determining values of model parameters. However transitions from old to new values were gradual and could take a few intervals before settling on the new model parameters.

The model tracking testbed control script (Section 6.3) was launched as outlined in Section 6.3.1. The LSTM models, Kalman filter, training data and tracking data generators were ran using Python in the same scripting environment as used in the primary system testbed (Section 6.2). The Tensorflow Tensorboard is used to provide summary statistics and track the training activity in real time.

Two approaches were taken for determining the $\mathrm{KF}$ measurement transform $\left(\mathrm{H}_{\mathrm{k}}\right.$ Matrix), with the second approach involving direct calculation proving to be more reliable:

1. Use of machine learning to detect changes in the input, learn and update the $\mathrm{H}_{\mathrm{k}}$ Matrix with learned coefficient, which produced an interesting but ineffective KF 
2. Calculate the $H_{k}$ Matrix from latest input measurements and the most recent estimated model state vector, which proved effective and was adopted for KF-LSTM

\subsubsection{Discovery Of KF Measurement Transform Coefficients Using LSTM}

In this section the LSTM is used to learn the parameters of the KF, this time including the $\mathrm{H}_{\mathrm{k}}$ Matrix, the Jacobian matrix of the model, here called the measurement transform. The $\mathrm{H}_{\mathrm{k}}$ matrix converges in around 6 training batches (16 seconds). However, this $\mathrm{H}_{\mathrm{k}}$ Matrix does not produce a very reliable $\mathrm{KF}$ which tracks the model closely. Hence a more analytical approach was used, as shown in the following section 8.5.2.

The training mean square error (difference between output and labels) of the measurement transform discovery LSTM unit are shown below. Convergence was defined empirically as training errors below a configured tolerance over a configured training window (number of consecutive iterations) with confidence. Convergence is then defined as $C_{w} \leq \varepsilon, \varepsilon=30$ for:

- Tolerance $\varepsilon=30$, at a confidence of $100 \%$, and

- $C_{w}$ the training cost of a set iterations in a sliding window, where cost is calculated as the mean square error of a training batch (see section 6.1.2.3.1) 


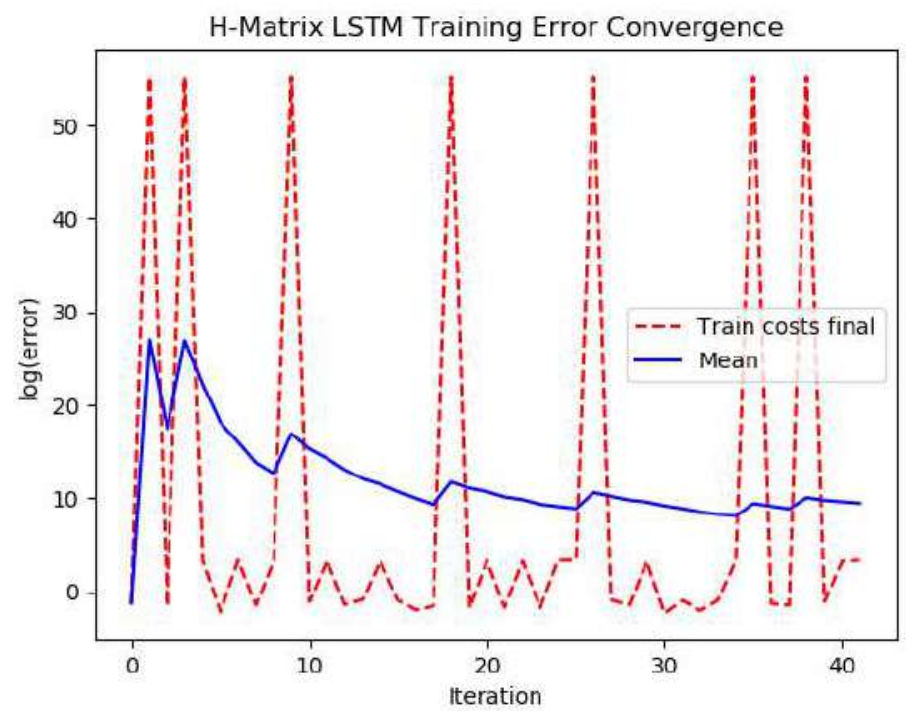

Figure 8.5.1: $\mathrm{H}_{\mathrm{k}}$ Matrix LSTM Training Error By Epoch

\subsubsection{Speed Of Convergence}

Convergence occurred fairly rapidly on this test bed using convergence criteria in the previous section. In this instance below it took 6 training batches on one epoch to converge (at a mean batch iteration time of 16 seconds on the test bed system).

\subsubsection{Direct Calculation Of EKF Measurement Transform}

In this section the $\mathrm{H}_{\mathrm{k}}$ matrix (\& its Jacobian) is calculated from the most recent measurements and model parameter vector. This is calculated as shown below: 
Given $\mathrm{z}=\mathrm{H}_{\mathrm{k}} \cdot \mathrm{x}, \quad=>\mathrm{H}_{\mathrm{k}}=\mathrm{h}(\mathrm{x})=\mathrm{z}\left(\mathrm{x}^{\mathrm{T}}\right)\left(\mathrm{x} \cdot \mathrm{x}^{\mathrm{T}}\right)^{-1}, \quad=>\mathrm{J}^{\mathrm{H}}=\frac{\Delta H}{\Delta x}=\frac{h\left(x_{2}\right)-h\left(x_{1}\right)}{x_{2}-x_{1}}$

where $\mathrm{J}^{\mathrm{H}}$ is the Jacobian of the $\mathrm{H}_{\mathrm{k}}$ matrix.

This is then used in the EKF along with LSTM-tuned Q and R matrices (as a variant of KF-LSTM1). This transformed KF-LSTM1 is used in the following sections, providing a stable KF able to track model parameters effectively.

\subsubsection{Evaluating KF-LSTM Tracking Behaviour}

This section shows model tracking for one LQN model coefficient, the number of users, using measurement data from the control unit, monitoring a test application and test client workload. The number of users is known ahead of time, and fully controllable, enabling model tracking testing and visualization.

The test data is generated as per the procedure below:

1. Generate a set of known LQN parameter vectors. The LQN parameters must include a single step change in value plus random noise.

2. Generate a random vector representing a single measurement and calculate its principal components.

3. The measurement data is the inverse principal component matrices of these noisy LQN parameters (using the principal components from step 2) multiplied by factor $m_{L S}$. NB: inverse principal component computation requires two inputs: a 
set of principal components (from the previous step), and a values vector to project (LQN parameters).

4. Kalman filter predictions are made from tracking the measurement values. The Kalman filter is setup to directly predict LQN parameters

5. These KF predictions are then compared to the known values from step 1).

The model tracking test yielded interesting results. In general, after learning the measurement transform matrix and its Jacobian, the Kalman filter was able to track the unknown system parameters $p$ fairly accurately. As illustrated in Figure 8.5.2, it was able to accommodate measurement noise and determine the model's unknown parameters vector.

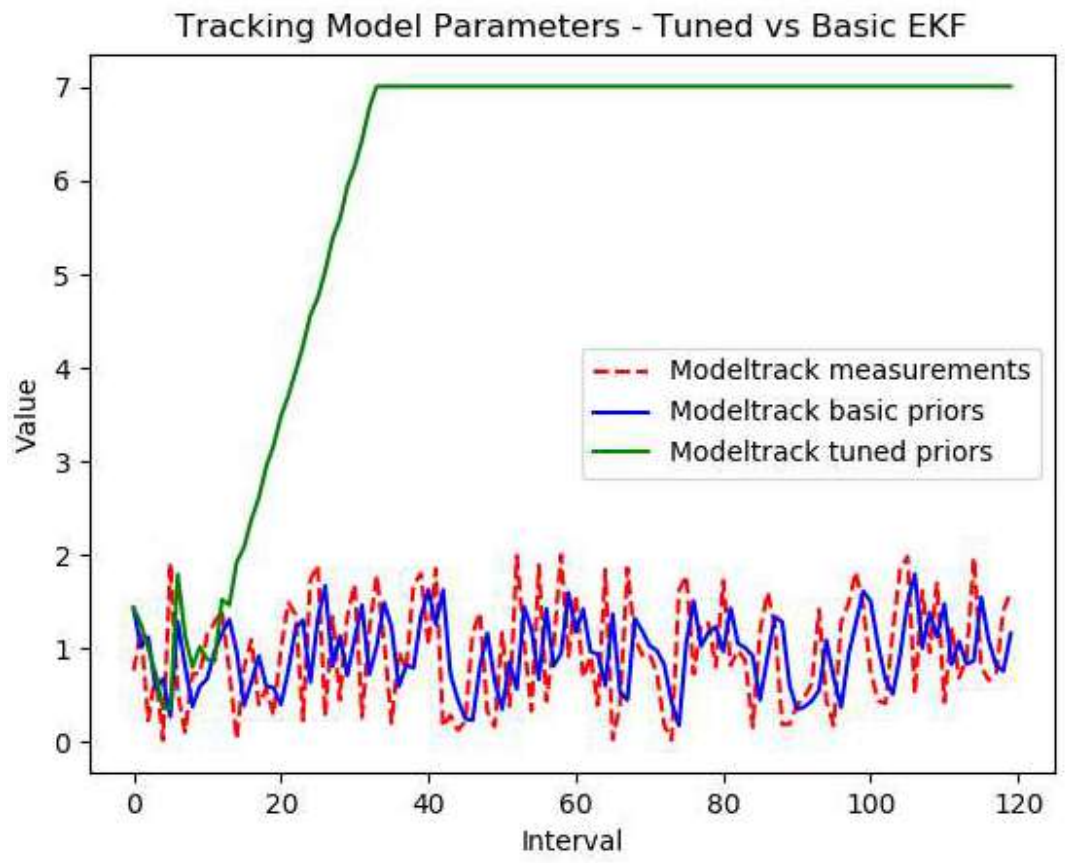

Figure 8.5.2: Model Tracking For No Of Users LQN Parameter (\# of Users = 7) 
In this first test, a noisy set of measurements are taken representing the measurables of the system under observation. The LSTM unit has been configured according to Section 6.1. It actively learns the unknown parameters of the model by doing a gradient descent on the error of the system solution around the unknown parameters (the error being calculated as in Section 6.1.2.3.1).

Once convergence has occurred, the discovered parameters are used to create a new measurement transform Jacobian matrix for the Kalman Filter. The now updated Kalman filter then searches for the target state via innovation updates until it reaches the best estimates corresponding to the noisy measurements.

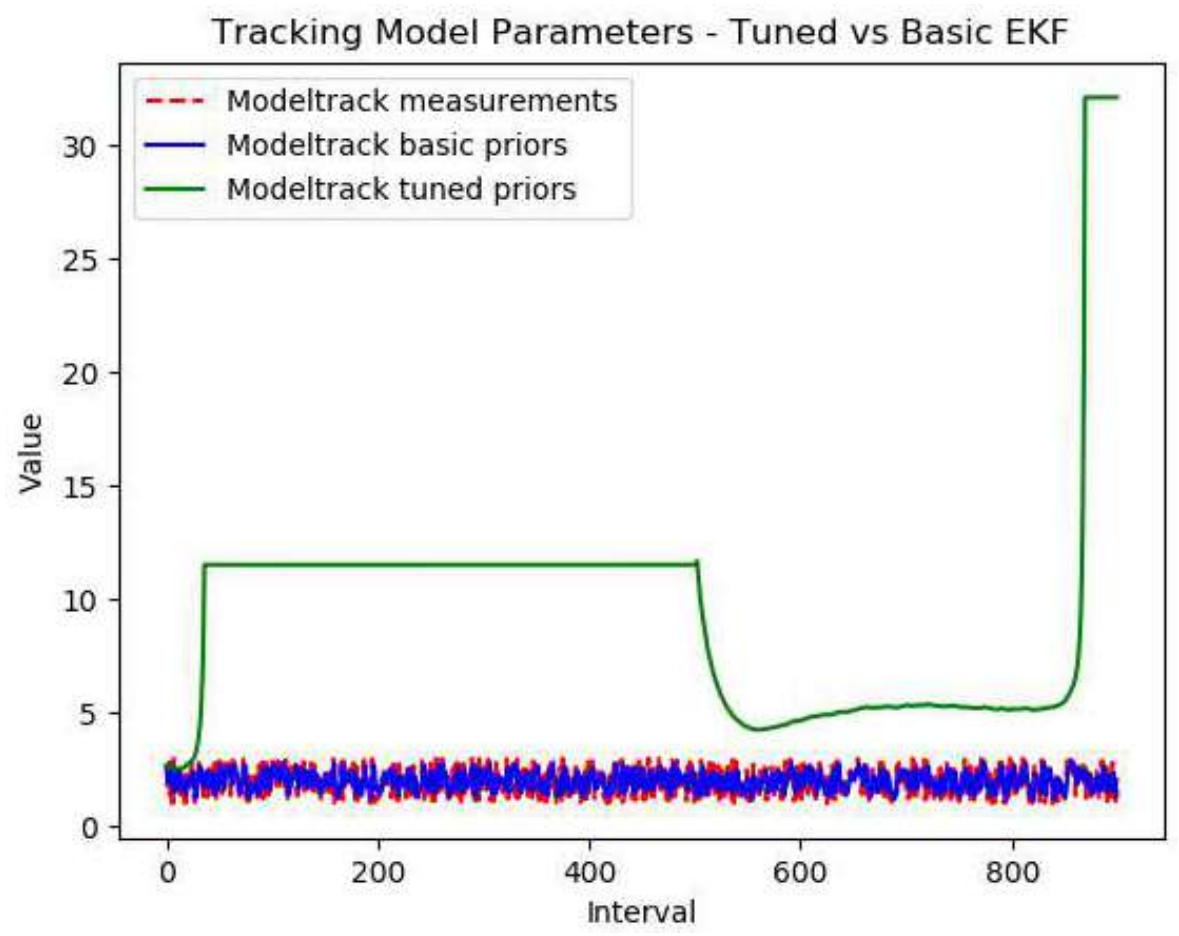

Figure 8.5.3: Model Tracking For No Of Users LQN Parameter (\# of Users = 12 \& 31) 
In this second test, the number of users in the tracked model changes from 12 to 31 . The Kalman filter first follows the algorithm in the previous example to learn the new unknown parameter vector, and then has its state converge to the values.

The number of users in the tracked model changes leading to more traffic on the observed system. The LSTM unit learns (via gradient descent convergence) of this change and updates the Kalman filter measurement transform's Jacobian function.

The Kalman filter state variables successfully converge to this new value after a delay of 400 iterations.

\subsection{Analysis Of KF-PCA-based Model Tracking Behaviour}

This section examines the KF-PCA algorithm in control mode, using test measurement data generated from the control unit to evaluate the KF-PCA algorithm's model tracking. It uses the KF-PCA algorithm outlined in chapter 4to estimate model parameters from measurement predictions from KF-LSTM1.

The test involved carrying out the following steps over multiple iterations, and collecting summary statistics:

1. Generate test data using the procedure in section 8.5 steps 1 to 3 .

2. Run KF-PCA.

Carrying on from chapter 4:

$$
\begin{aligned}
& \mathrm{n}=\text { number of principal components, } \\
& \mathrm{x}_{\mathrm{k}}=\text { Kalman filter's current state }
\end{aligned}
$$


$\mathrm{x}_{\mathrm{k}-1}=$ Kalman filter state prediction prior to update,

$\boldsymbol{\sigma}^{\mathrm{xk}}=$ Covariance matrix for $\mathrm{x}_{\mathrm{k}}$, each entry is a row to column covariance

$\mathrm{PCA}^{\sigma \mathrm{x}}=$ Principal components matrix for covariance matrix $\boldsymbol{\sigma}^{\mathrm{xk}}$

Therefore implement dimensionality reduction as follows:

Given $P C A^{\sigma x}=\left\{v_{1} . . v_{n}\right\}$ of eigenvectors $v_{i}$

define $P C A^{\sigma x l}=\left\{v_{1} . . v_{l}\right\} \cup\left\{\varnothing_{1} . . \varnothing_{n-l}\right\}, l<n \quad\left(\&\right.$ null vectors $\left.\varnothing_{j}\right)$

substitute $P C A^{\sigma x}=P C A^{\sigma x l}$ (in the PCA algorithm of chapter 4)

3. The resulting KF-PCA estimates are then compared to the known values from step 1).

The results of the above steps are shown below. The test measurement data typically involved a transition/value-change at time step 50. Some definitions:

lqnp: LQN Parameters,

msmts: Measurements from control unit

kfpriors: Measurements KF Prior $\left(\mathrm{x}_{\mathrm{k}-1}\right)$

lqn-kfprior: LQN estimate using PCA (using kfpriors as input)

lqnerrors: LQN Error 

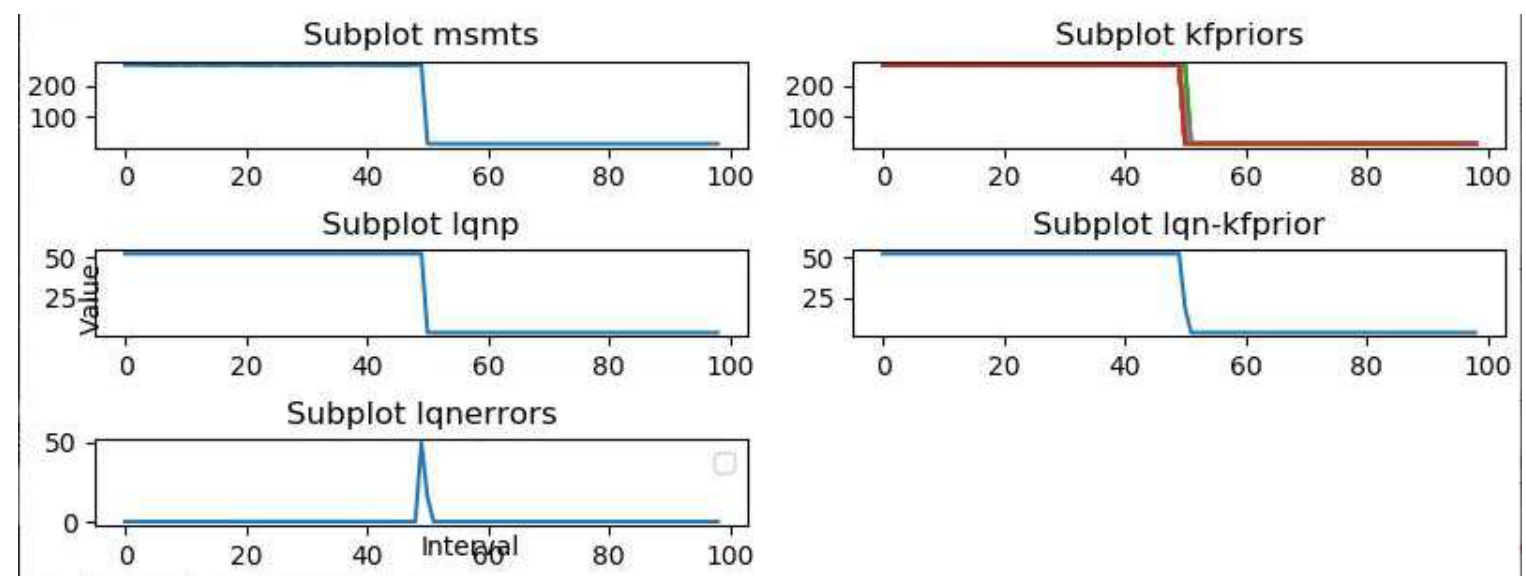

Figure 8.6.0.1: LQN Parameter(lqnp), measurements \& KF Prior \& Error- Experiment 1
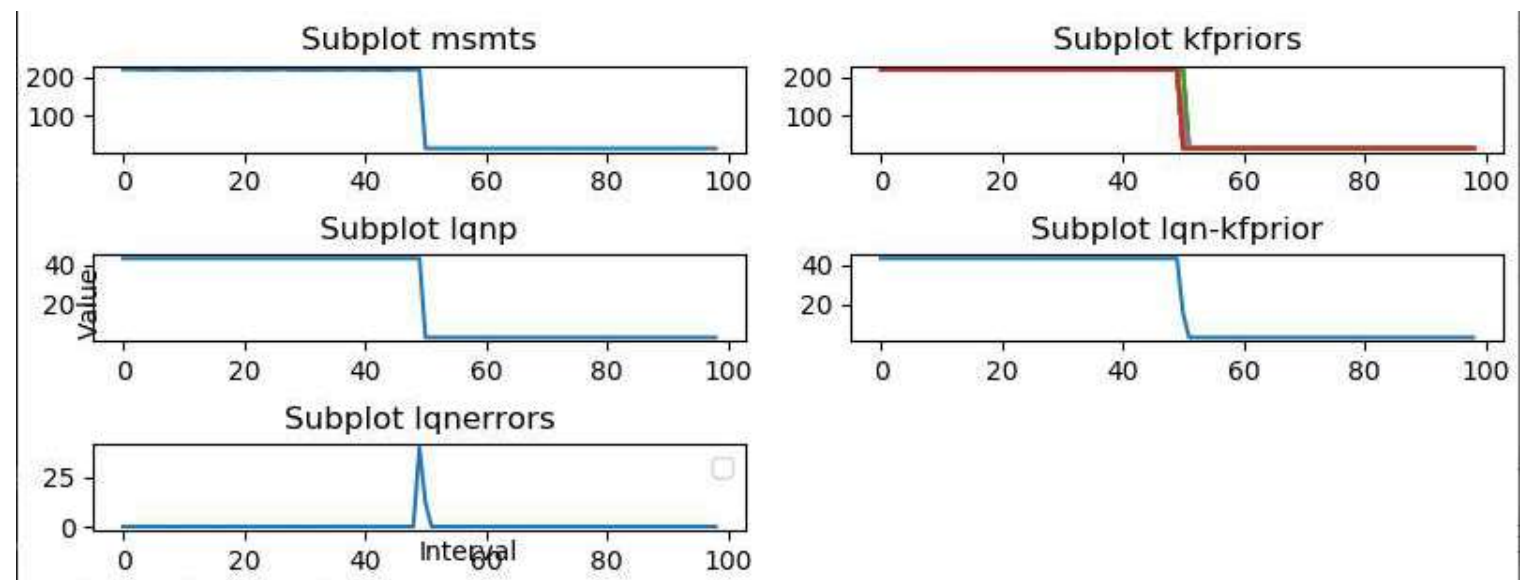

Figure 8.6.0.2: LQN Parameter(lqnp), measurements \& KF Prior \& Error- Experiment 2 

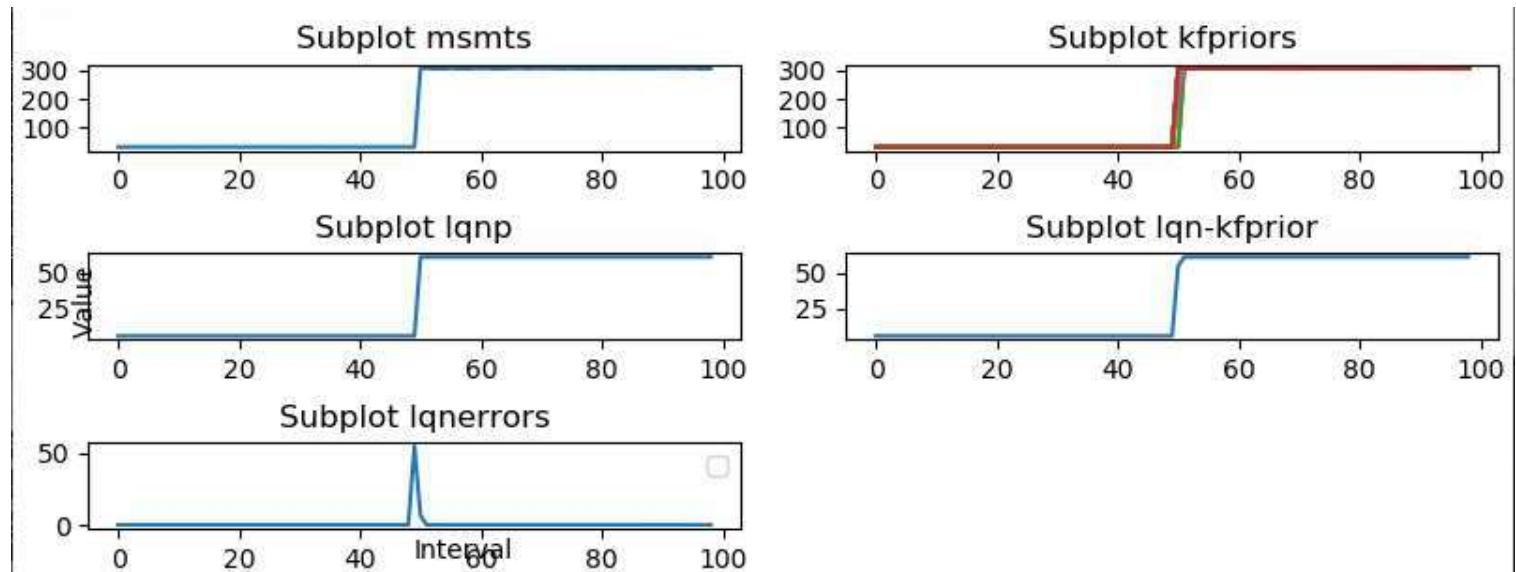

Figure 8.6.0.3: LQN Parameter(p), measurements \& KF Prior \& Error- Experiment 3

The above 3 plots represent the first 3 experiments (out of 10) done for PCA testing.

Their percentage mean square errors are reasonable for the data tested $(0.13028 \%$, $0.11412 \%$ and $0.038575 \%$ respectively).
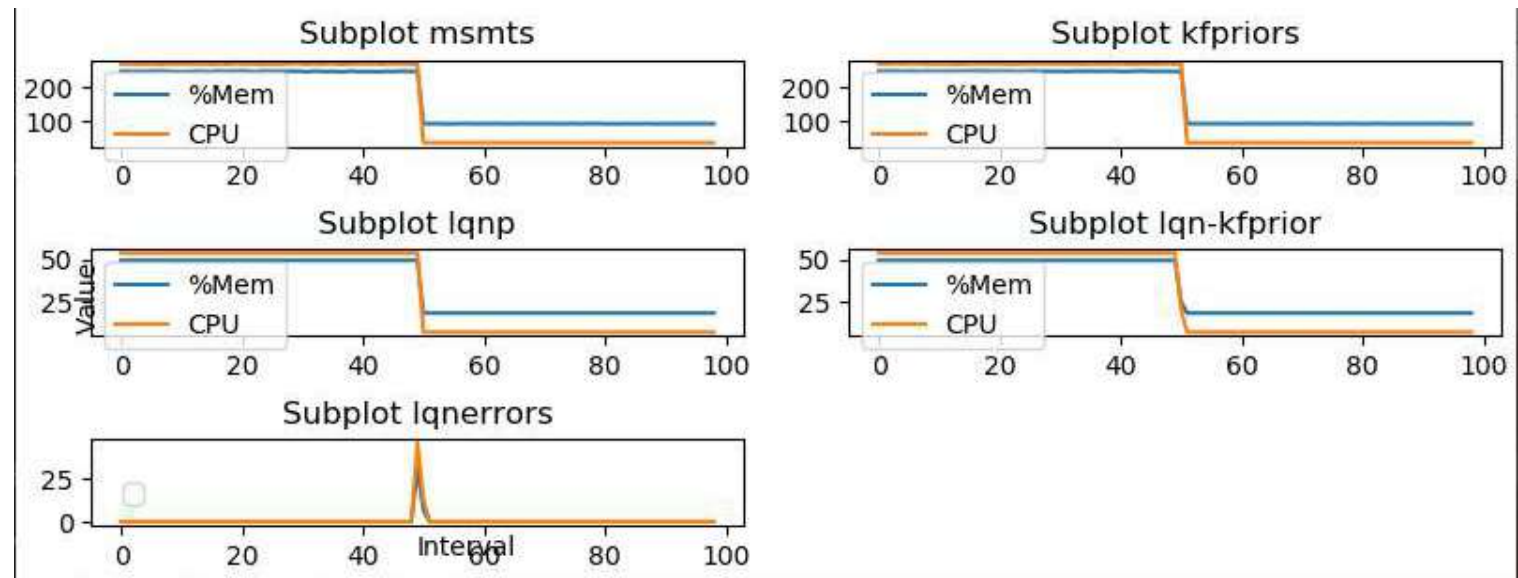

Figure 8.6.0.4: KF-PCA LQN Parameters, measurements \& KF Prior \& Error This plot above represents tracking of multiple model parameters, with showing low tracking error $(0.0670 \%$ and $0.1051 \%$ for $\mathrm{p}[0]$ and $\mathrm{p}[1])$. 


\subsubsection{Measurement data $\left(z_{k}\right)$}

Below is the measurement data for the KF-PCA-based model tracking analysis in section 8.6.

\begin{tabular}{|c|c|c|c|c|c|c|c|c|c|c|c|c|}
\hline k & $z_{k}(0)$ & $z_{k}(1)$ & $z_{k}(2)$ & $z_{k}(3)$ & $z_{k}(4)$ & $z_{k}(5)$ & $z_{k}(6)$ & $z_{k}(7)$ & $z_{k}(8)$ & $z_{k}(9)$ & $z_{k}(10)$ & $z_{k}(11)$ \\
\hline 1 & 16.71 & 21.63 & 17.56 & 20.51 & 17.06 & 17.67 & 16.98 & 17.85 & 18.95 & 19.98 & 17.10 & 21.14 \\
\hline 2 & 19.30 & 17.16 & 19.07 & 16.69 & 18.64 & 19.44 & 18.13 & 19.63 & 17.84 & 20.16 & 16.78 & 20.27 \\
\hline 3 & 17.44 & 17.92 & 20.55 & 17.90 & 21.25 & 18.21 & 16.73 & 18.59 & 21.59 & 18.68 & 18.94 & 17.96 \\
\hline 4 & 20.03 & 18.59 & 18.21 & 20.64 & 21.29 & 17.46 & 17.91 & 20.82 & 21.43 & 17.15 & 21.12 & 20.57 \\
\hline 5 & 21.43 & 21.27 & 19.00 & 20.70 & 20.50 & 19.74 & 18.44 & 17.15 & 17.00 & 18.68 & 21.47 & 18.36 \\
\hline 6 & 17.39 & 17.76 & 20.73 & 18.86 & 20.34 & 17.41 & 17.56 & 20.56 & 17.57 & 19.74 & 19.49 & 18.60 \\
\hline 7 & 18.70 & 19.94 & 18.09 & 20.15 & 19.01 & 18.03 & 19.92 & 18.15 & 19.19 & 18.07 & 18.76 & 16.98 \\
\hline 8 & 16.98 & 20.57 & 20.57 & 19.13 & 18.83 & 19.21 & 18.47 & 18.12 & 16.71 & 21.61 & 20.34 & 16.68 \\
\hline 9 & 16.85 & 18.35 & 17.66 & 20.82 & 17.35 & 18.40 & 19.94 & 19.93 & 21.20 & 20.56 & 19.45 & 17.76 \\
\hline 10 & 19.76 & 18.71 & 20.38 & 19.33 & 17.94 & 19.58 & 16.65 & 20.30 & 17.78 & 20.97 & 20.65 & 21.27 \\
\hline 11 & 18.72 & 17.20 & 18.83 & 19.55 & 19.39 & 17.70 & 20.53 & 17.09 & 18.48 & 20.45 & 21.02 & 17.53 \\
\hline 12 & 20.98 & 19.17 & 18.85 & 17.93 & 17.48 & 20.98 & 20.04 & 20.36 & 20.01 & 20.23 & 17.29 & 20.00 \\
\hline 13 & 19.79 & 20.13 & 21.38 & 18.12 & 17.61 & 17.35 & 21.16 & 20.29 & 17.38 & 18.04 & 20.74 & 18.43 \\
\hline 14 & 20.22 & 17.28 & 18.04 & 20.04 & 17.79 & 18.31 & 18.74 & 18.04 & 16.70 & 18.66 & 16.78 & 20.27 \\
\hline 15 & 21.46 & 18.13 & 18.46 & 18.52 & 17.78 & 18.19 & 19.54 & 17.22 & 18.92 & 20.77 & 18.88 & 17.30 \\
\hline
\end{tabular}




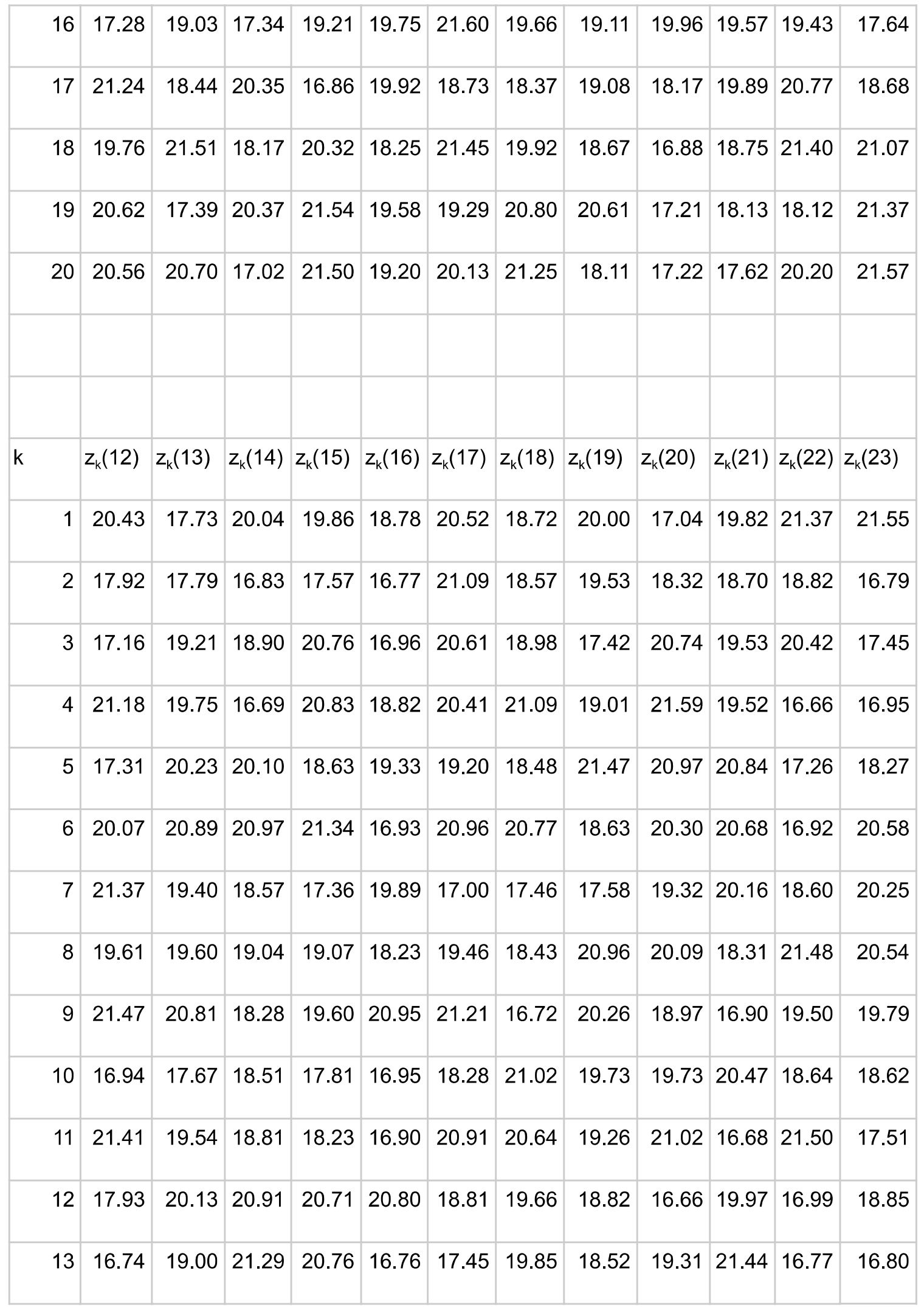




\begin{tabular}{|r|r|r|r|r|r|r|r|r|r|r|r|r|}
\hline 14 & 18.74 & 16.82 & 18.90 & 17.67 & 20.14 & 21.02 & 18.49 & 20.46 & 16.93 & 19.90 & 20.05 & 17.40 \\
\hline 15 & 17.73 & 17.96 & 20.15 & 18.62 & 19.31 & 17.63 & 20.16 & 19.89 & 18.05 & 20.92 & 21.38 & 18.45 \\
\hline 16 & 16.68 & 17.91 & 17.76 & 17.12 & 17.61 & 20.35 & 18.90 & 17.07 & 21.02 & 19.47 & 19.15 & 17.84 \\
\hline 17 & 17.57 & 19.16 & 20.06 & 17.80 & 17.17 & 19.41 & 21.26 & 20.08 & 20.83 & 21.37 & 17.67 & 19.88 \\
\hline 18 & 17.17 & 20.78 & 18.37 & 19.67 & 19.09 & 16.82 & 20.49 & 16.96 & 18.10 & 19.35 & 19.21 & 17.85 \\
\hline 19 & 18.13 & 21.29 & 21.23 & 18.57 & 16.73 & 21.44 & 17.39 & 17.80 & 17.84 & 20.11 & 18.87 & 18.37 \\
\hline 20 & 16.87 & 17.49 & 19.73 & 18.32 & 20.62 & 20.38 & 20.37 & 19.68 & 20.94 & 20.08 & 20.13 & 17.38 \\
\hline
\end{tabular}

\subsubsection{LQN parameters, real $(p)$ and predicted $\left(x_{k-1}\right)$}

$\% \operatorname{Error}\left(\mathrm{p}_{\mathrm{k}}\right)=\sum_{i=1}^{N=3} \frac{\left(x_{k \mid k-1}(i)-p_{k}(i)\right)}{N \cdot p_{k}(i)} \times 100$

\begin{tabular}{|r|r|r|r|r|r|r|r|r|}
\hline \multicolumn{2}{|l|}{ Average \%Error(p): } & 0.26 & & & & \\
\hline 1 & 48.51 & 31.86 & 92.49 & 48.16 & 31.86 & 92.49 & -0.35 & 0.24 \\
\hline 2 & 48.51 & 31.86 & 92.49 & 48.00 & 31.86 & 92.49 & -0.51 & 0.35 \\
\hline 3 & 48.51 & 31.86 & 92.49 & 48.32 & 31.86 & 92.49 & -0.20 & 0.13 \\
\hline 4 & 48.51 & 31.86 & 92.49 & 48.09 & 31.86 & 92.49 & -0.42 & 0.29 \\
\hline 5 & 48.51 & 31.86 & 92.49 & 48.58 & 31.86 & 92.49 & 0.07 & 0.05 \\
\hline 6 & 48.51 & 31.86 & 92.49 & 48.36 & 31.86 & 92.49 & -0.16 & 0.11 \\
\hline 7 & 48.51 & 31.86 & 92.49 & 48.49 & 31.86 & 92.49 & -0.02 & 0.02 \\
\hline & & & & & & & & \\
\hline 1 & & & & & & & & \\
\hline
\end{tabular}




\begin{tabular}{|c|c|c|c|c|c|c|c|c|}
\hline 8 & 48.51 & 31.86 & 92.49 & 48.05 & 31.86 & 92.49 & -0.46 & 0.32 \\
\hline 9 & 48.51 & 31.86 & 92.49 & 48.14 & 31.86 & 92.49 & -0.37 & 0.26 \\
\hline 10 & 48.51 & 31.86 & 92.49 & 47.94 & 31.86 & 92.49 & -0.57 & 0.39 \\
\hline 11 & 48.51 & 31.86 & 92.49 & 47.75 & 31.86 & 92.49 & -0.76 & 0.52 \\
\hline 12 & 48.51 & 31.86 & 92.49 & 48.62 & 31.86 & 92.49 & 0.11 & 0.08 \\
\hline 13 & 48.51 & 31.86 & 92.49 & 48.41 & 31.86 & 92.49 & -0.11 & 0.07 \\
\hline 14 & 48.51 & 31.86 & 92.49 & 47.94 & 31.86 & 92.49 & -0.57 & 0.39 \\
\hline 15 & 48.51 & 31.86 & 92.49 & 48.17 & 31.86 & 92.49 & -0.34 & 0.23 \\
\hline 16 & 48.51 & 31.86 & 92.49 & 48.66 & 31.86 & 92.49 & 0.15 & 0.10 \\
\hline 17 & 48.51 & 31.86 & 92.49 & 47.96 & 31.86 & 92.49 & -0.56 & 0.38 \\
\hline 18 & 48.51 & 31.86 & 92.49 & 48.05 & 31.86 & 92.49 & -0.47 & 0.32 \\
\hline 19 & 48.51 & 31.86 & 92.49 & 48.39 & 31.86 & 92.49 & -0.12 & 0.08 \\
\hline 20 & 48.51 & 31.86 & 92.49 & 47.32 & 31.86 & 92.49 & -1.19 & 0.82 \\
\hline 47 & 48.51 & 31.86 & 92.49 & 48.67 & 31.86 & 92.49 & 0.16 & 0.11 \\
\hline 48 & 48.51 & 31.86 & 92.49 & 47.74 & 31.86 & 92.49 & -0.78 & 0.53 \\
\hline 49 & 48.51 & 31.86 & 92.49 & 48.70 & 31.86 & 92.49 & 0.19 & 0.13 \\
\hline 50 & 48.51 & 31.86 & 92.49 & 47.91 & 31.86 & 92.49 & -0.60 & 0.41 \\
\hline 51 & 57.38 & 66.00 & 66.96 & 57.27 & 66.00 & 66.96 & -0.12 & 0.07 \\
\hline 52 & 57.38 & 66.00 & 66.96 & 56.95 & 66.00 & 66.96 & -0.43 & 0.25 \\
\hline 53 & 57.38 & 66.00 & 66.96 & 57.22 & 66.00 & 66.96 & -0.16 & 0.09 \\
\hline 54 & 57.38 & 66.00 & 66.96 & 56.56 & 66.00 & 66.96 & -0.82 & 0.48 \\
\hline
\end{tabular}




\begin{tabular}{|c|c|c|c|c|c|c|c|c|}
\hline 55 & 57.38 & 66.00 & 66.96 & 58.03 & 66.00 & 66.96 & 0.65 & 0.38 \\
\hline 56 & 57.38 & 66.00 & 66.96 & 56.75 & 66.00 & 66.96 & -0.63 & 0.37 \\
\hline 57 & 57.38 & 66.00 & 66.96 & 57.12 & 66.00 & 66.96 & -0.26 & 0.15 \\
\hline 58 & 57.38 & 66.00 & 66.96 & 57.00 & 66.00 & 66.96 & -0.38 & 0.22 \\
\hline 59 & 57.38 & 66.00 & 66.96 & 57.23 & 66.00 & 66.96 & -0.15 & 0.09 \\
\hline 60 & 57.38 & 66.00 & 66.96 & 56.87 & 66.00 & 66.96 & -0.52 & 0.30 \\
\hline 61 & 57.38 & 66.00 & 66.96 & 56.58 & 66.00 & 66.96 & -0.80 & 0.47 \\
\hline 62 & 57.38 & 66.00 & 66.96 & 57.07 & 66.00 & 66.96 & -0.31 & 0.18 \\
\hline 63 & 57.38 & 66.00 & 66.96 & 57.53 & 66.00 & 66.96 & 0.14 & 0.08 \\
\hline 64 & 57.38 & 66.00 & 66.96 & 57.10 & 66.00 & 66.96 & -0.28 & 0.16 \\
\hline 65 & 57.38 & 66.00 & 66.96 & 56.85 & 66.00 & 66.96 & -0.53 & 0.31 \\
\hline 66 & 57.38 & 66.00 & 66.96 & 57.30 & 66.00 & 66.96 & -0.08 & 0.05 \\
\hline 67 & 57.38 & 66.00 & 66.96 & 57.44 & 66.00 & 66.96 & 0.06 & 0.04 \\
\hline 68 & 57.38 & 66.00 & 66.96 & 56.02 & 66.00 & 66.96 & -1.36 & 0.79 \\
\hline 69 & 57.38 & 66.00 & 66.96 & 57.54 & 66.00 & 66.96 & 0.15 & 0.09 \\
\hline 70 & 57.38 & 66.00 & 66.96 & 57.50 & 66.00 & 66.96 & 0.12 & 0.07 \\
\hline 71 & 57.38 & 66.00 & 66.96 & 56.42 & 66.00 & 66.96 & -0.96 & 0.56 \\
\hline 72 & 57.38 & 66.00 & 66.96 & 57.58 & 66.00 & 66.96 & 0.20 & 0.12 \\
\hline 73 & 57.38 & 66.00 & 66.96 & 56.90 & 66.00 & 66.96 & -0.48 & 0.28 \\
\hline 74 & 57.38 & 66.00 & 66.96 & 56.84 & 66.00 & 66.96 & -0.55 & 0.32 \\
\hline 75 & 57.38 & 66.00 & 66.96 & 57.40 & 66.00 & 66.96 & 0.02 & 0.01 \\
\hline
\end{tabular}




\begin{tabular}{|c|c|c|c|c|c|c|c|c|}
\hline 76 & 57.38 & 66.00 & 66.96 & 56.88 & 66.00 & 66.96 & -0.50 & 0.29 \\
\hline 77 & 57.38 & 66.00 & 66.96 & 57.82 & 66.00 & 66.96 & 0.43 & 0.25 \\
\hline 78 & 57.38 & 66.00 & 66.96 & 56.74 & 66.00 & 66.96 & -0.65 & 0.38 \\
\hline 91 & 57.38 & 66.00 & 66.96 & 56.91 & 66.00 & 66.96 & -0.47 & 0.27 \\
\hline 92 & 57.38 & 66.00 & 66.96 & 56.19 & 66.00 & 66.96 & -1.19 & 0.69 \\
\hline 93 & 57.38 & 66.00 & 66.96 & 57.68 & 66.00 & 66.96 & 0.29 & 0.17 \\
\hline 94 & 57.38 & 66.00 & 66.96 & 57.06 & 66.00 & 66.96 & -0.32 & 0.19 \\
\hline 95 & 57.38 & 66.00 & 66.96 & 56.74 & 66.00 & 66.96 & -0.64 & 0.37 \\
\hline 96 & 57.38 & 66.00 & 66.96 & 57.40 & 66.00 & 66.96 & 0.02 & 0.01 \\
\hline 97 & 57.38 & 66.00 & 66.96 & 56.63 & 66.00 & 66.96 & -0.75 & 0.44 \\
\hline 98 & 57.38 & 66.00 & 66.96 & 56.84 & 66.00 & 66.96 & -0.55 & 0.32 \\
\hline 99 & 57.38 & 66.00 & 66.96 & 56.63 & 66.00 & 66.96 & -0.75 & 0.44 \\
\hline 100 & 57.38 & 66.00 & 66.96 & 57.42 & 66.00 & 66.96 & 0.04 & 0.02 \\
\hline
\end{tabular}


The summary for all experiments is shown below:
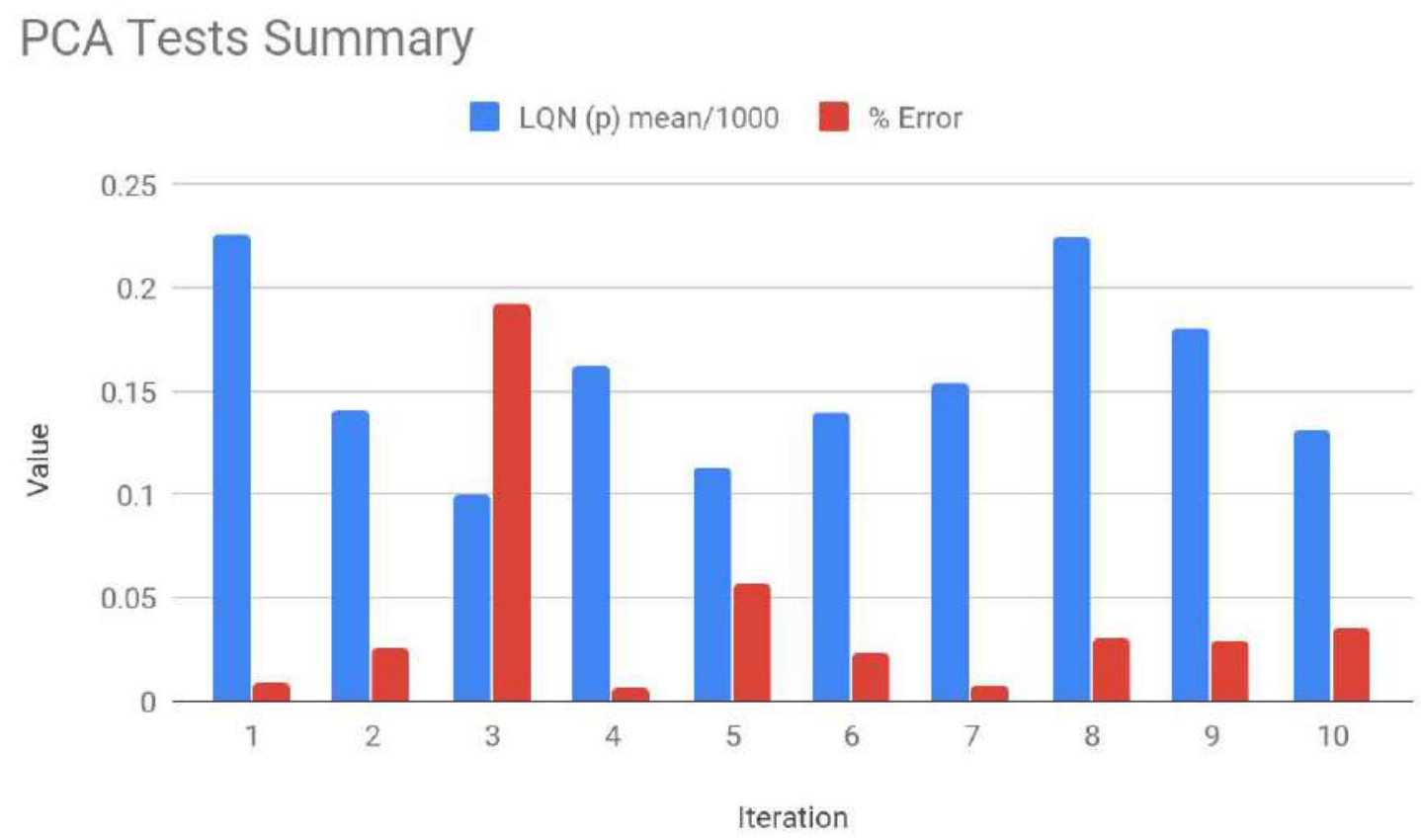

Figure 8.6.0.5: Summary LQN Parameter Error Percentages Over All Experiments

From figure 8.6.0.5, the error on all LQN parameter coordinates is less than $0.20 \%$ on all 10 experiments $($ mean error $=0.04167 \%$, variance $=0.5467 \%)$. The procedure is quite robust to noise due to the eigenspace projection. $90 \%$ of the experiments had error rates less than $0.05 \%$.

The robustness to noise is enhanced by using a dimensionality reduction [98] procedure. Less significant eigenvector dimensions are discarded to operate in a lower-dimensional subspace. 


\subsubsection{Comparison Of KF-PCA-based and Non-KF-PCA Model Tracking}

This section examines the KF-LSTM1 algorithm in control mode, using test measurement data generated from the control unit to directly perform model tracking. This is for the purpose of comparison to the KF-PCA algorithm in the previous section. Overall the KF-PCA algorithm has a slight edge in terms of accuracy, with the differences apparent in time steps where there are value changes in the tracked model parameters.

A follow on estimation of LQN parameters using KF-LSTM1 was done. This was for the purposes of comparison to the KF-PCA LQN parameter inference algorithm. The test involved carrying out the following steps over multiple experiments, and collecting summary statistics:

1. Generate test data following the procedure in section 8.5.

2. The error values are then compared to the test in the previous section 8.6 The results of the above steps are shown below. As in the previous section, the test measurement data typically involved a transition/value-change at time step 50 . Variables in graphs are defined in section 8.6. 

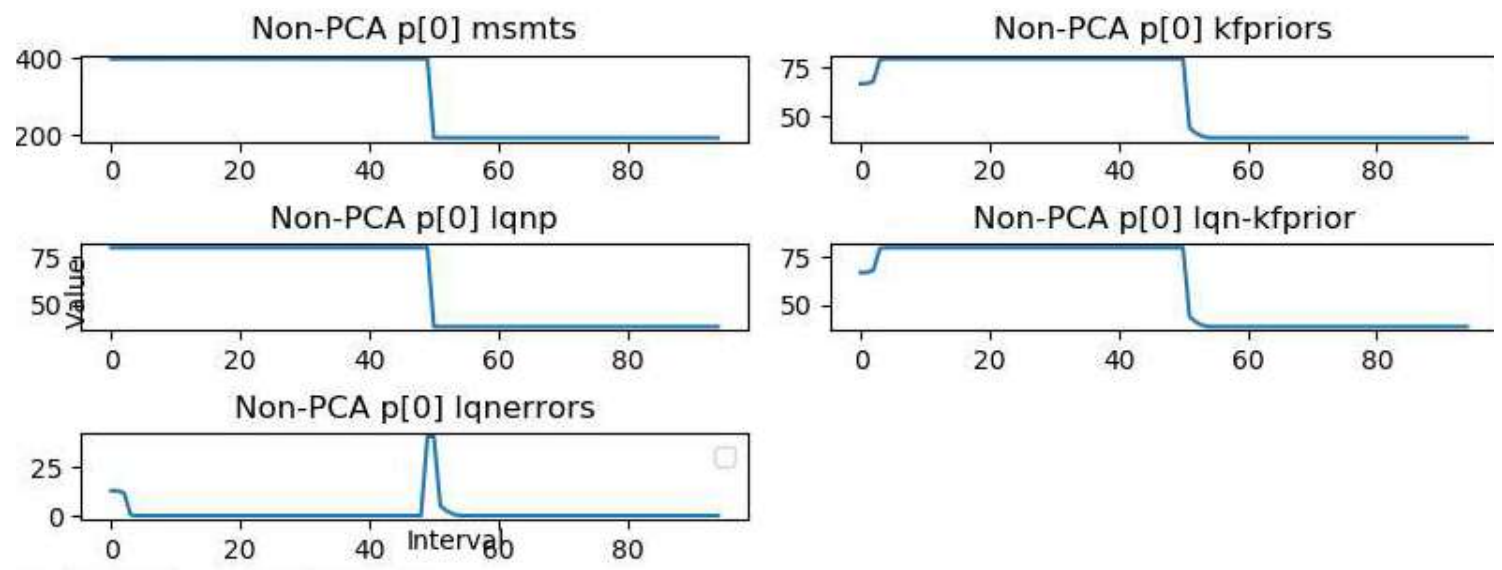

Figure 8.6.1.0: Non-KF-PCA LQN Parameters \& KF Prior \& Error - Experiment 1
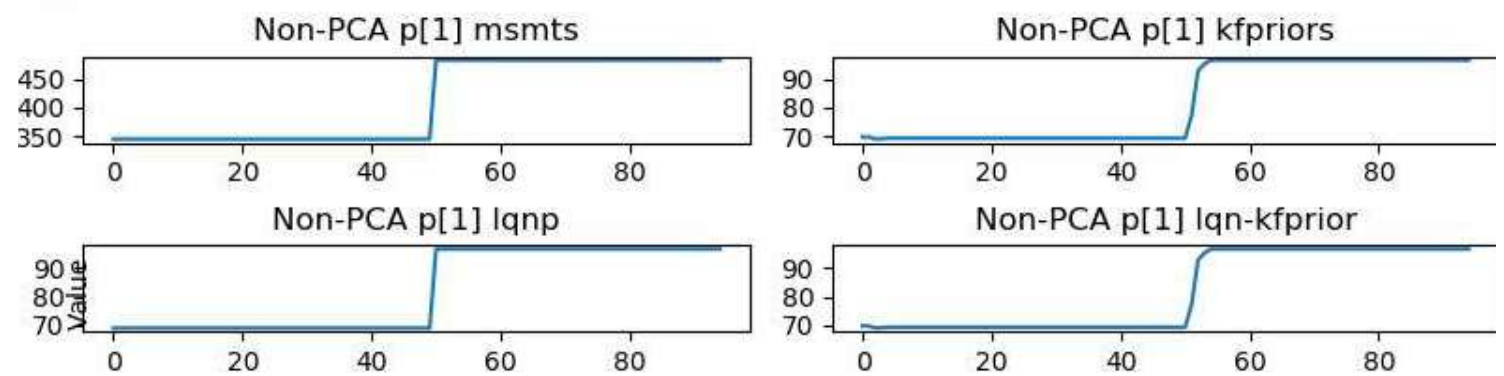

Non-PCA p[1] Iqnerrors

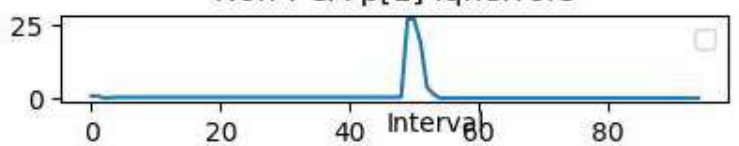

Figure 8.6.1.1: Non-KF-PCA LQN Parameter \& KF Prior \& Error - Experiment 2 

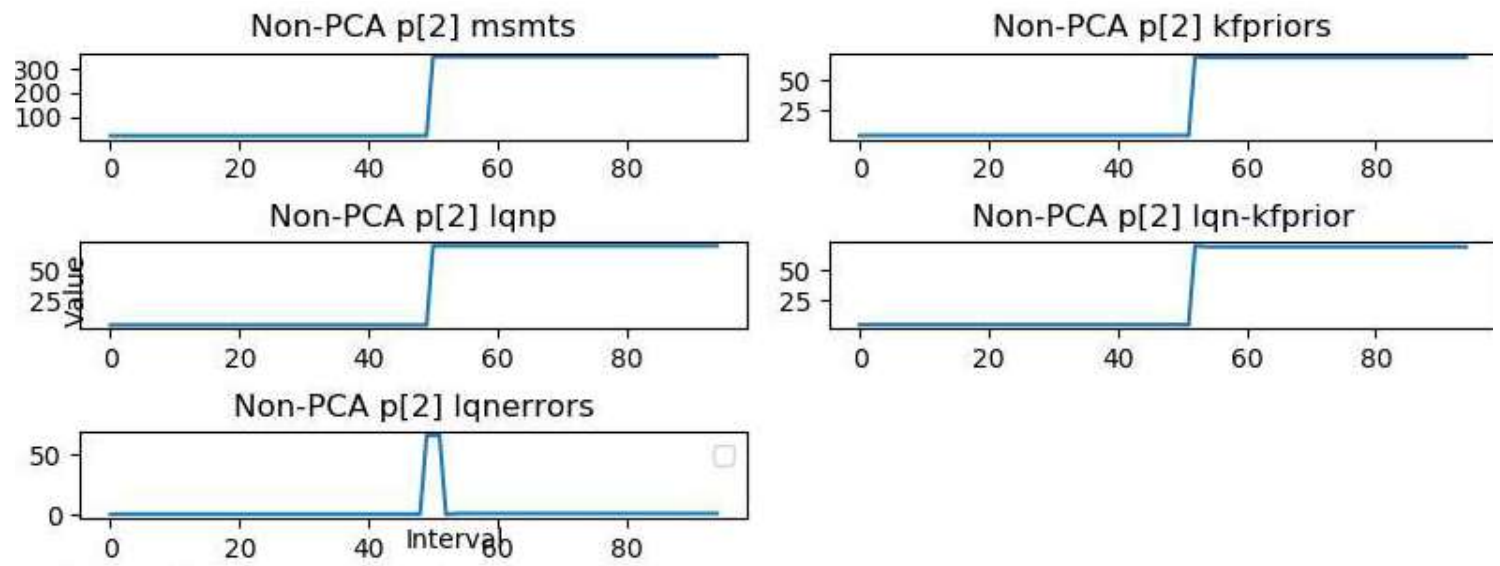

Figure 8.6.1.2: Non-KF-PCA LQN Parameter \& KF Prior \& Error - Experiment 3
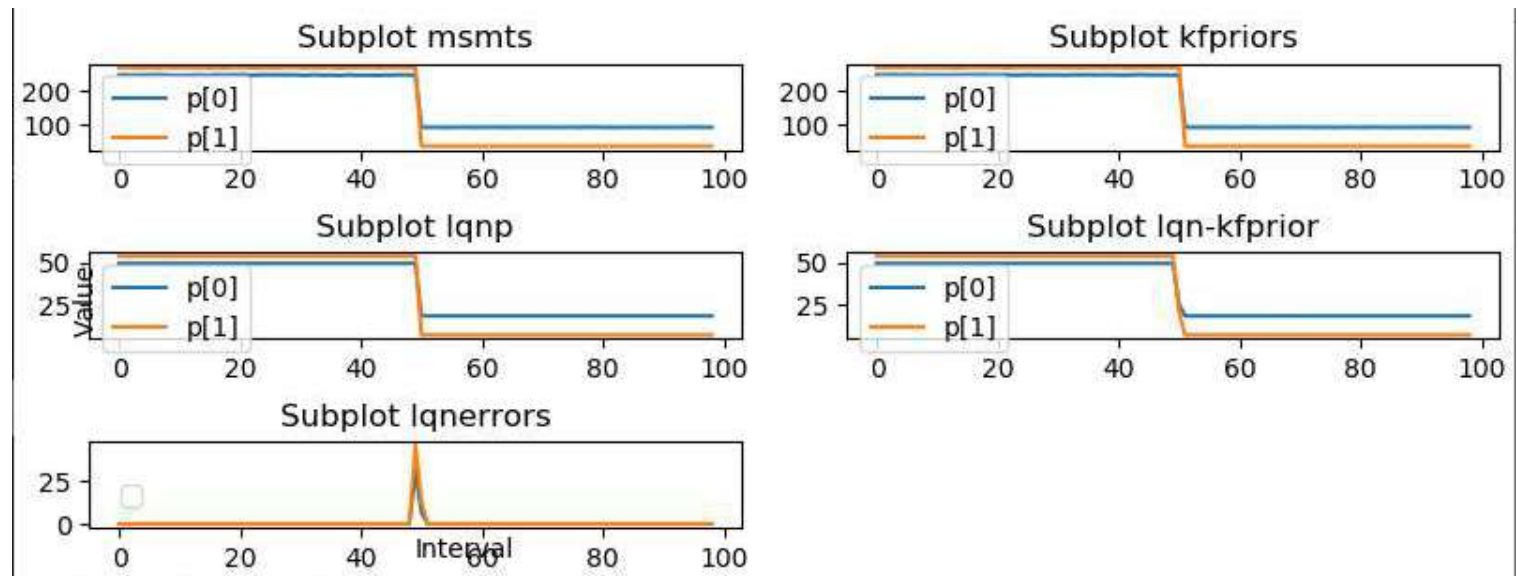

Figure 8.6.1.3: Non-KF-PCA LQN Parameters (p [0] \& p [1]) \& KF Prior \& Error This plot above represents non-KF-PCA tracking of multiple model parameters, with showing higher tracking error than KF-PCA-based tracking (28.85\% and $56.96 \%$ for $\mathrm{p}$ [0] and $\mathrm{p}[1]$ versus $0.0670 \%$ and $0.1051 \%$ respectively).

Key samples of the raw data are shown below: 


\subsubsection{Measurement data $\left(\mathrm{z}_{\mathrm{k}}\right)$}

Below is the measurement data for the KF-PCA to non-KF-PCA model tracking comparison in section 8.6.2.

\begin{tabular}{|c|c|c|c|c|c|c|c|c|c|c|c|c|}
\hline $\mathrm{k}$ & $z_{k}(0)$ & $z_{k}(1)$ & $z_{k}(2)$ & $z_{k}(3)$ & $z_{k}(4)$ & $z_{k}(5)$ & $z_{k}(6)$ & $z_{k}(7)$ & $z_{k}(8)$ & $z_{k}(9)$ & $z_{k}(10)$ & $z_{k}(11)$ \\
\hline 1 & 4.31 & 4.97 & 7.64 & 5.56 & 3.73 & 4.14 & 5.51 & 4.24 & 7.17 & 6.07 & 5.84 & 5.19 \\
\hline 2 & 5.37 & 6.08 & 7.78 & 4.47 & 6.43 & 8.16 & 7.96 & 4.11 & 5.67 & 4.03 & 3.91 & 8.15 \\
\hline 3 & 4.46 & 5.17 & 5.23 & 7.65 & 7.93 & 5.69 & 4.72 & 6.79 & 7.64 & 6.79 & 6.00 & 7.49 \\
\hline 4 & 6.01 & 5.45 & 7.35 & 4.61 & 7.18 & 3.78 & 4.47 & 8.01 & 8.23 & 6.92 & 4.43 & 4.96 \\
\hline 5 & 3.92 & 4.12 & 6.31 & 7.07 & 7.50 & 4.87 & 5.84 & 4.41 & 8.08 & 6.97 & 5.02 & 8.17 \\
\hline 6 & 5.33 & 7.88 & 7.68 & 7.50 & 7.44 & 3.71 & 7.35 & 6.38 & 7.19 & 7.01 & 6.86 & 5.04 \\
\hline 7 & 7.04 & 7.12 & 4.80 & 4.00 & 5.27 & 3.81 & 7.04 & 8.06 & 6.88 & 7.74 & 6.98 & 6.02 \\
\hline 8 & 4.81 & 6.78 & 6.26 & 8.30 & 4.13 & 7.50 & 6.47 & 4.46 & 6.44 & 6.22 & 3.73 & 6.76 \\
\hline 9 & 5.11 & 8.58 & 3.73 & 4.89 & 4.92 & 6.75 & 7.97 & 5.79 & 5.82 & 6.58 & 4.55 & 7.52 \\
\hline 10 & 5.77 & 5.41 & 8.08 & 6.02 & 8.28 & 3.69 & 6.93 & 8.48 & 4.39 & 5.87 & 4.91 & 4.42 \\
\hline 11 & 5.52 & 5.14 & 7.99 & 5.84 & 6.83 & 5.04 & 4.54 & 4.80 & 7.40 & 7.21 & 5.75 & 4.84 \\
\hline 12 & 4.08 & 6.46 & 5.01 & 7.63 & 7.01 & 5.77 & 4.99 & 7.81 & 3.70 & 8.47 & 7.32 & 8.03 \\
\hline 13 & 5.42 & 3.82 & 5.76 & 4.05 & 7.86 & 7.87 & 7.91 & 8.37 & 3.76 & 4.20 & 7.50 & 7.90 \\
\hline 14 & 4.31 & 8.56 & 8.25 & 4.16 & 4.10 & 8.44 & 5.29 & 5.34 & 6.79 & 4.49 & 7.84 & 4.50 \\
\hline 15 & 4.94 & 3.72 & 8.26 & 3.69 & 4.23 & 7.95 & 7.88 & 5.99 & 4.14 & 4.52 & 4.72 & 5.38 \\
\hline 16 & 6.67 & 3.98 & 4.59 & 7.46 & 6.82 & 6.59 & 5.58 & 8.15 & 5.51 & 8.33 & 7.52 & 4.74 \\
\hline 17 & 4.70 & 7.00 & 5.33 & 4.74 & 6.33 & 7.12 & 5.82 & 6.11 & 5.80 & 4.29 & 8.59 & 8.33 \\
\hline
\end{tabular}




\begin{tabular}{|c|c|c|c|c|c|c|c|c|c|c|c|c|}
\hline 18 & 6.13 & 8.02 & 5.21 & 4.89 & 5.38 & 7.31 & 8.21 & 4.70 & 6.61 & 4.93 & 6.64 & 5.78 \\
\hline 19 & 7.80 & 5.39 & 7.96 & 5.64 & 3.67 & 7.69 & 5.90 & 6.94 & 4.31 & 7.35 & 5.87 & 8.24 \\
\hline 20 & 6.63 & 3.90 & 4.79 & 4.03 & 8.13 & 4.47 & 6.52 & 3.66 & 8.54 & 5.48 & 4.28 & 4.33 \\
\hline k & $z_{k}(12)$ & $z_{k}(13)$ & $z_{k}(14)$ & $z_{k}(15)$ & $z_{k}(16)$ & $z_{k}(17)$ & $z_{k}(18)$ & $z_{k}(19)$ & $z_{k}(20)$ & $z_{k}(21)$ & $z_{k}(22)$ & $z_{k}(23)$ \\
\hline 1 & 5.17 & 6.13 & 6.31 & 6.52 & 7.84 & 4.82 & 5.81 & 6.14 & 6.25 & 8.43 & 7.26 & 7.68 \\
\hline 2 & 5.58 & 4.58 & 7.97 & 5.23 & 4.79 & 4.23 & 4.54 & 6.53 & 5.96 & 4.75 & 5.38 & 8.34 \\
\hline 3 & 8.19 & 4.44 & 6.58 & 7.74 & 6.07 & 3.67 & 6.50 & 8.51 & 5.06 & 7.12 & 5.64 & 7.34 \\
\hline 4 & 7.33 & 6.91 & 5.64 & 8.05 & 6.15 & 5.79 & 6.59 & 7.94 & 8.08 & 7.65 & 4.04 & 4.05 \\
\hline 5 & 8.11 & 5.61 & 5.28 & 7.99 & 5.74 & 7.54 & 4.94 & 4.70 & 4.76 & 4.07 & 8.16 & 6.80 \\
\hline 6 & 7.23 & 6.45 & 5.81 & 6.75 & 6.71 & 4.74 & 5.39 & 7.80 & 6.18 & 8.38 & 7.68 & 5.23 \\
\hline 7 & 5.54 & 4.55 & 7.98 & 5.38 & 6.47 & 4.19 & 4.74 & 6.19 & 6.66 & 4.64 & 3.92 & 6.80 \\
\hline 8 & 7.58 & 5.14 & 7.33 & 6.37 & 7.84 & 3.66 & 5.41 & 7.76 & 4.69 & 4.58 & 7.86 & 5.76 \\
\hline 9 & 8.00 & 6.65 & 6.40 & 6.16 & 4.55 & 6.77 & 7.25 & 5.86 & 4.94 & 7.67 & 6.10 & 4.25 \\
\hline 10 & 7.94 & 6.70 & 8.52 & 7.51 & 5.09 & 7.02 & 6.20 & 6.19 & 6.46 & 7.87 & 6.04 & 8.40 \\
\hline 11 & 6.95 & 7.36 & 3.95 & 5.40 & 6.84 & 5.54 & 3.92 & 6.54 & 6.87 & 7.38 & 7.01 & 6.11 \\
\hline 12 & 6.23 & 8.12 & 5.66 & 7.28 & 5.34 & 4.11 & 5.20 & 4.06 & 8.40 & 6.79 & 6.03 & 7.33 \\
\hline 13 & 4.70 & 8.48 & 7.64 & 7.92 & 8.38 & 8.14 & 4.32 & 7.13 & 7.44 & 7.42 & 4.17 & 6.80 \\
\hline 14 & 6.08 & 3.73 & 5.17 & 5.16 & 5.24 & 7.74 & 5.33 & 7.13 & 7.15 & 7.16 & 3.81 & 7.79 \\
\hline 15 & 5.89 & 4.99 & 8.32 & 4.75 & 4.90 & 5.73 & 4.42 & 4.77 & 6.28 & 6.82 & 4.10 & 7.23 \\
\hline 16 & 7.19 & 6.20 & 5.68 & 4.57 & 6.15 & 7.17 & 5.10 & 5.92 & 7.04 & 8.22 & 7.74 & 6.63 \\
\hline
\end{tabular}




\begin{tabular}{|r|r|r|r|r|r|r|r|r|r|r|r|r|}
\hline 17 & 6.84 & 8.39 & 8.52 & 8.07 & 6.45 & 7.88 & 6.15 & 4.10 & 6.14 & 5.26 & 7.53 & 3.78 \\
\hline 18 & 8.31 & 7.71 & 4.32 & 5.77 & 4.03 & 6.92 & 4.97 & 5.67 & 6.14 & 6.72 & 6.01 & 5.99 \\
\hline 19 & 3.69 & 8.39 & 7.03 & 8.39 & 8.10 & 5.20 & 3.65 & 7.83 & 6.68 & 6.55 & 6.74 & 7.02 \\
\hline 20 & 5.38 & 6.26 & 8.60 & 4.79 & 6.38 & 4.32 & 4.98 & 4.57 & 5.27 & 6.28 & 7.72 & 6.79 \\
\hline
\end{tabular}

\subsubsection{LQN parameters, real (p) and predicted $\left(x_{k-1}\right)$}

$\% \operatorname{Error}\left(\mathrm{p}_{\mathrm{k}}\right)$ is defined in eq(22)

\begin{tabular}{|c|c|c|c|c|c|c|c|c|}
\hline \multicolumn{2}{|c|}{ Average \%Error(p): } & \multirow{2}{*}{$\begin{array}{r}12.97 \\
p_{k}(1)\end{array}$} & \multirow[b]{2}{*}{$p_{k}(2)$} & \multirow[b]{2}{*}{$x_{k-1}(0)$} & \multirow[b]{2}{*}{$x_{k-1}(1)$} & \multirow[b]{2}{*}{$x_{k-1}(2)$} & \multirow[b]{2}{*}{$\operatorname{Error}\left(\mathrm{p}_{\mathrm{k}}(0)\right)$} & \multirow[b]{2}{*}{$\%$ Error $\left(p_{k}\right)$} \\
\hline k & $p_{k}(0)$ & & & & & & & \\
\hline 1 & 72.49 & 71.60 & 33.85 & 72.49 & 71.60 & 33.85 & 0.00 & 0.00 \\
\hline 2 & 72.49 & 71.60 & 33.85 & 75.54 & 73.57 & 36.81 & 3.05 & 5.24 \\
\hline 3 & 72.49 & 71.60 & 33.85 & 75.57 & 73.66 & 36.80 & 3.08 & 5.29 \\
\hline 4 & 72.49 & 71.60 & 33.85 & 75.62 & 73.74 & 36.82 & 3.13 & 5.36 \\
\hline 5 & 72.49 & 71.60 & 33.85 & 75.66 & 73.80 & 36.84 & 3.17 & 5.43 \\
\hline 6 & 72.49 & 71.60 & 33.85 & 75.67 & 73.82 & 36.84 & 3.18 & 5.44 \\
\hline 7 & 72.49 & 71.60 & 33.85 & 75.69 & 73.84 & 36.85 & 3.20 & 5.48 \\
\hline 8 & 72.49 & 71.60 & 33.85 & 75.71 & 73.87 & 36.87 & 3.22 & 5.51 \\
\hline 9 & 72.49 & 71.60 & 33.85 & 75.73 & 73.89 & 36.88 & 3.24 & 5.54 \\
\hline 10 & 72.49 & 71.60 & 33.85 & 75.76 & 73.93 & 36.90 & 3.27 & 5.60 \\
\hline 11 & 72.49 & 71.60 & 33.85 & 75.79 & 73.96 & 36.92 & 3.30 & 5.64 \\
\hline 12 & 72.49 & 71.60 & 33.85 & 75.82 & 73.99 & 36.93 & 3.32 & 5.68 \\
\hline
\end{tabular}




\begin{tabular}{|c|c|c|c|c|c|c|c|c|}
\hline 13 & 72.49 & 71.60 & 33.85 & 75.84 & 74.02 & 36.95 & 3.35 & 5.72 \\
\hline 14 & 72.49 & 71.60 & 33.85 & 75.88 & 74.07 & 36.98 & 3.39 & 5.80 \\
\hline 15 & 72.49 & 71.60 & 33.85 & 75.93 & 74.13 & 37.01 & 3.44 & 5.88 \\
\hline 16 & 72.49 & 71.60 & 33.85 & 75.99 & 74.20 & 37.06 & 3.50 & 5.98 \\
\hline 17 & 72.49 & 71.60 & 33.85 & 76.05 & 74.26 & 37.09 & 3.56 & 6.07 \\
\hline 18 & 72.49 & 71.60 & 33.85 & 76.10 & 74.32 & 37.13 & 3.61 & 6.16 \\
\hline 19 & 72.49 & 71.60 & 33.85 & 76.12 & 74.35 & 37.15 & 3.63 & 6.20 \\
\hline 20 & 72.49 & 71.60 & 33.85 & 76.15 & 74.38 & 37.17 & 3.66 & 6.25 \\
\hline 47 & 72.49 & 71.60 & 33.85 & 79.65 & 78.20 & 39.69 & 7.16 & 12.12 \\
\hline 48 & 72.49 & 71.60 & 33.85 & 79.90 & 78.48 & 39.88 & 7.41 & 12.55 \\
\hline 49 & 72.49 & 71.60 & 33.85 & 80.14 & 78.74 & 40.05 & 7.65 & 12.95 \\
\hline 50 & 72.49 & 71.60 & 33.85 & 80.41 & 79.02 & 40.24 & 7.92 & 13.39 \\
\hline 51 & 99.46 & 99.72 & 57.73 & 80.61 & 79.24 & 40.39 & -18.86 & 23.18 \\
\hline 52 & 99.46 & 99.72 & 57.73 & 80.98 & 79.64 & 40.66 & -18.49 & 22.76 \\
\hline 53 & 99.46 & 99.72 & 57.73 & 81.06 & 79.73 & 40.74 & -18.40 & 22.66 \\
\hline 54 & 99.46 & 99.72 & 57.73 & 81.17 & 79.85 & 40.84 & -18.29 & 22.52 \\
\hline 55 & 99.46 & 99.72 & 57.73 & 81.31 & 80.00 & 40.96 & -18.16 & 22.36 \\
\hline 56 & 99.46 & 99.72 & 57.73 & 81.41 & 80.11 & 41.06 & -18.06 & 22.23 \\
\hline 57 & 99.46 & 99.72 & 57.73 & 81.51 & 80.22 & 41.15 & -17.95 & 22.10 \\
\hline 58 & 99.46 & 99.72 & 57.73 & 81.63 & 80.36 & 41.26 & -17.83 & 21.96 \\
\hline 59 & 99.46 & 99.72 & 57.73 & 81.74 & 80.47 & 41.36 & -17.73 & 21.82 \\
\hline
\end{tabular}




\begin{tabular}{|c|c|c|c|c|c|c|c|c|}
\hline 60 & 99.46 & 99.72 & 57.73 & 81.84 & 80.59 & 41.46 & -17.62 & 21.70 \\
\hline 61 & 99.46 & 99.72 & 57.73 & 81.97 & 80.72 & 41.57 & -17.50 & 21.54 \\
\hline 62 & 99.46 & 99.72 & 57.73 & 82.11 & 80.88 & 41.71 & -17.35 & 21.36 \\
\hline 63 & 99.46 & 99.72 & 57.73 & 82.20 & 80.98 & 41.79 & -17.26 & 21.25 \\
\hline 64 & 99.46 & 99.72 & 57.73 & 82.35 & 81.14 & 41.93 & -17.12 & 21.07 \\
\hline 65 & 99.46 & 99.72 & 57.73 & 82.52 & 81.32 & 42.08 & -16.95 & 20.86 \\
\hline 66 & 99.46 & 99.72 & 57.73 & 82.62 & 81.44 & 42.18 & -16.84 & 20.73 \\
\hline 67 & 99.46 & 99.72 & 57.73 & 82.75 & 81.57 & 42.29 & -16.72 & 20.58 \\
\hline 68 & 99.46 & 99.72 & 57.73 & 82.88 & 81.71 & 42.41 & -16.59 & 20.42 \\
\hline 69 & 99.46 & 99.72 & 57.73 & 83.07 & 81.92 & 42.59 & -16.40 & 20.19 \\
\hline 70 & 99.46 & 99.72 & 57.73 & 83.26 & 82.13 & 42.77 & -16.20 & 19.95 \\
\hline 71 & 99.46 & 99.72 & 57.73 & 83.40 & 82.29 & 42.90 & -16.07 & 19.78 \\
\hline 72 & 99.46 & 99.72 & 57.73 & 83.58 & 82.48 & 43.06 & -15.89 & 19.56 \\
\hline 73 & 99.46 & 99.72 & 57.73 & 83.71 & 82.63 & 43.19 & -15.75 & 19.39 \\
\hline 74 & 99.46 & 99.72 & 57.73 & 83.87 & 82.80 & 43.33 & -15.59 & 19.19 \\
\hline 75 & 99.46 & 99.72 & 57.73 & 84.04 & 82.98 & 43.49 & -15.42 & 18.98 \\
\hline 76 & 99.46 & 99.72 & 57.73 & 84.26 & 83.22 & 43.69 & -15.20 & 18.71 \\
\hline 77 & 99.46 & 99.72 & 57.73 & 84.49 & 83.47 & 43.90 & -14.98 & 18.44 \\
\hline 78 & 99.46 & 99.72 & 57.73 & 84.67 & 83.67 & 44.07 & -14.80 & 18.21 \\
\hline 79 & 99.46 & 99.72 & 57.73 & 84.92 & 83.94 & 44.30 & -14.54 & 17.90 \\
\hline 91 & 99.46 & 99.72 & 57.73 & 88.95 & 88.32 & 48.02 & -10.51 & 12.94 \\
\hline
\end{tabular}




\begin{tabular}{|c|c|c|c|c|c|c|c|c|}
\hline 92 & 99.46 & 99.72 & 57.73 & 89.58 & 89.00 & 48.61 & -9.88 & 12.16 \\
\hline 93 & 99.46 & 99.72 & 57.73 & 90.38 & 89.87 & 49.34 & -9.08 & 11.18 \\
\hline 94 & 99.46 & 99.72 & 57.73 & 91.65 & 91.25 & 50.52 & -7.81 & 9.61 \\
\hline 95 & 99.46 & 99.72 & 57.73 & 92.19 & 91.83 & 51.01 & -7.28 & 8.96 \\
\hline 96 & 99.46 & 99.72 & 57.73 & 99.46 & 99.72 & 57.73 & 0.00 & 0.00 \\
\hline 97 & 99.46 & 99.72 & 57.73 & 99.37 & 99.61 & 57.64 & -0.09 & 0.12 \\
\hline 98 & 99.46 & 99.72 & 57.73 & 99.27 & 99.50 & 57.54 & -0.20 & 0.24 \\
\hline 99 & 99.46 & 99.72 & 57.73 & 99.15 & 99.38 & 57.44 & -0.31 & 0.39 \\
\hline 100 & 99.46 & 99.72 & 57.73 & 99.02 & 99.23 & 57.32 & -0.45 & 0.55 \\
\hline
\end{tabular}




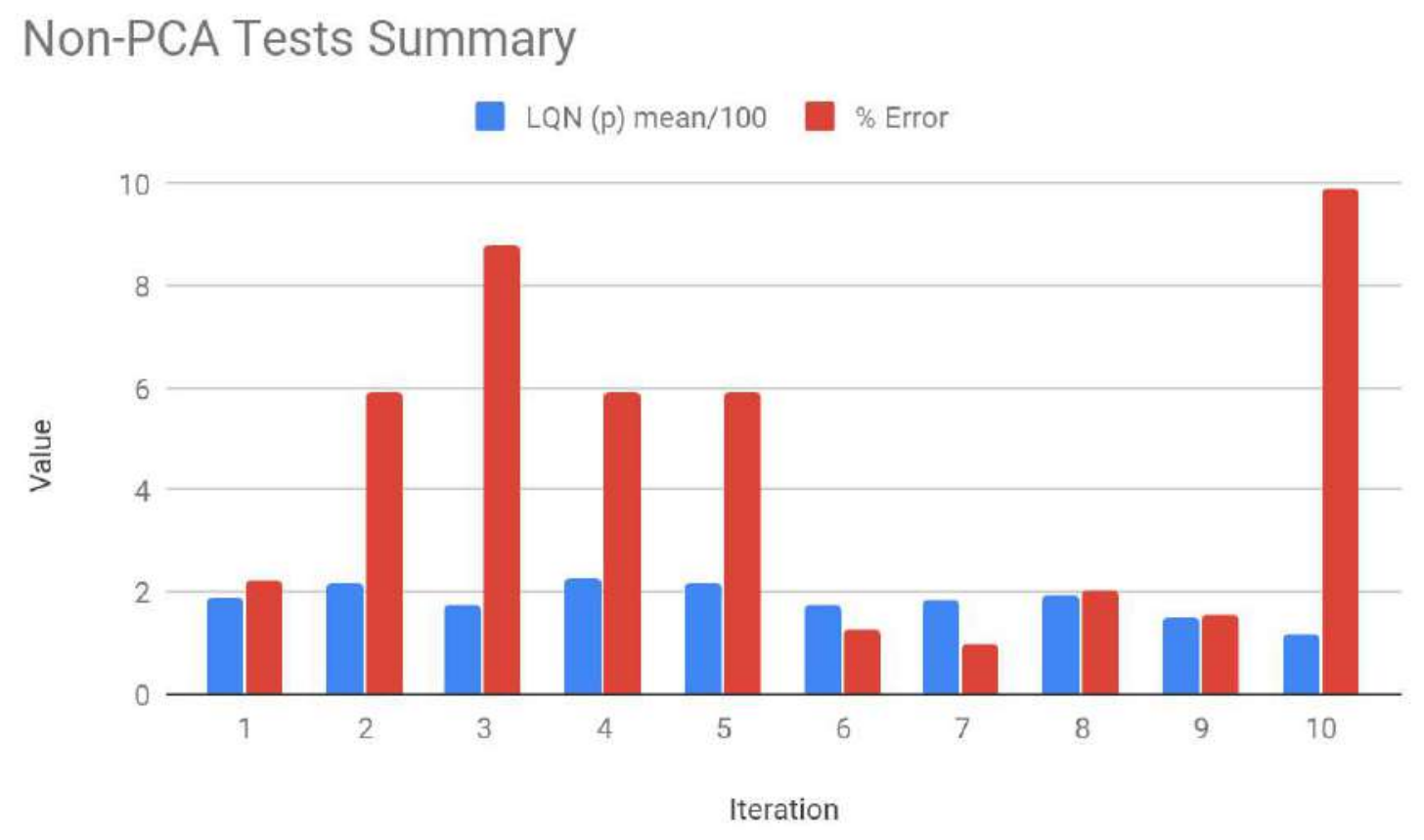

Figure 8.6.1.4: Summary Of Non-PCA LQN Error Percentages Over All Experiments From figure 8.6.1.4, the mean error on all LQN parameter coordinates is $4.463 \%$ (std = $3.187 \%$ ) on all experiments.

The variance in absolute error is $10.72 \%$ here (figure 8.6 .1 .4 ) compared to $0.5467 \%$ in the KF-PCA-based tests (figure 8.6.0.5).

This shows a reduction in sensitivity to noise in the measurements, mainly due to the post-processing of Kalman filter predictions. The KF-PCA inference algorithm helps eliminate noise by using the eigenspace of the measurements, and by frequent updating of the eigenspace to LQN parameter projection factor $\left(m_{L S}\right.$ in section 4.2). The lower variance due to noise is dependent on the strength of correlation of the underlying pattern 
of the data with respect to noise correlation. In cases where noise correlation is high (of the same order of magnitude or higher), the KF-PCA algorithm is likely to suffer from divergence in its predictions.

In a qualitative sense, there are smoother transitions between values in the vanilla Kalman filter state variable. This is quite evident in figure 9.6.1.1 with transition curves clearly visible. The KF-PCA-based Kalman filter has sharper transitions indicating closer tracking of measurement variables. The KF-PCA-based algorithm also shows sharp spikes in the error values where noise in measurements can cause perturbations in the eigenspace scores which causes periodic errors in the inferences.

Using the most significant eigenvector subspace rather than the whole eigenspace is a good approach to noise sensitivity reduction, and prior knowledge of the structure in the data can be used in further reducing feature set for the smallest possible lower-dimensional subspace. This makes the algorithm more attractive. PCA-based Kalman filter is a lot simpler and easier to implement, as it does not require a custom state transition matrix, measurement matrix or measurement Jacobian to capture the model of each variable to be tracked.

\section{7 $\quad$ System Testbed Analysis}

The research goal of evaluating the control system's impact on a real application is tackled this system testbed analysis. This section examines the controller's control unit in 
control mode, using measurement data generated from the test client (Section 6.2.2) to evaluate active and passive control (Section 6.1.4.1) performance. The system testbed (Section 6.2) was launched using the deployment scheme outlined in Section 6.2.0. The test client was also launched, embedded in the traffic generator (Section 6.2.3). This enabled both active and passive control modes to be run with nearly identical environmental parameters, ensuring consistency in comparative analysis. Overall there is a reduction in mean and standard deviation of testbed application client response times for the controller in active control mode vis a vis passive control mode (Section 6.1.4.1).

\subsubsection{Active \& Passive Monitor Impact}

A control system is running in active control mode. The workload includes 10000 search client requests at a rate of 1 per ms. Control actions are triggered and issued by the monitor. From the logs, it appears the monitor enrolled a new node to the search engine cluster when the configured utilization threshold was crossed using the ADDREPLICA command (Section 6.1.5.1). It then removed the replica once utilization returned below the threshold. The cycle was repeated again whenever the threshold was crossed. 


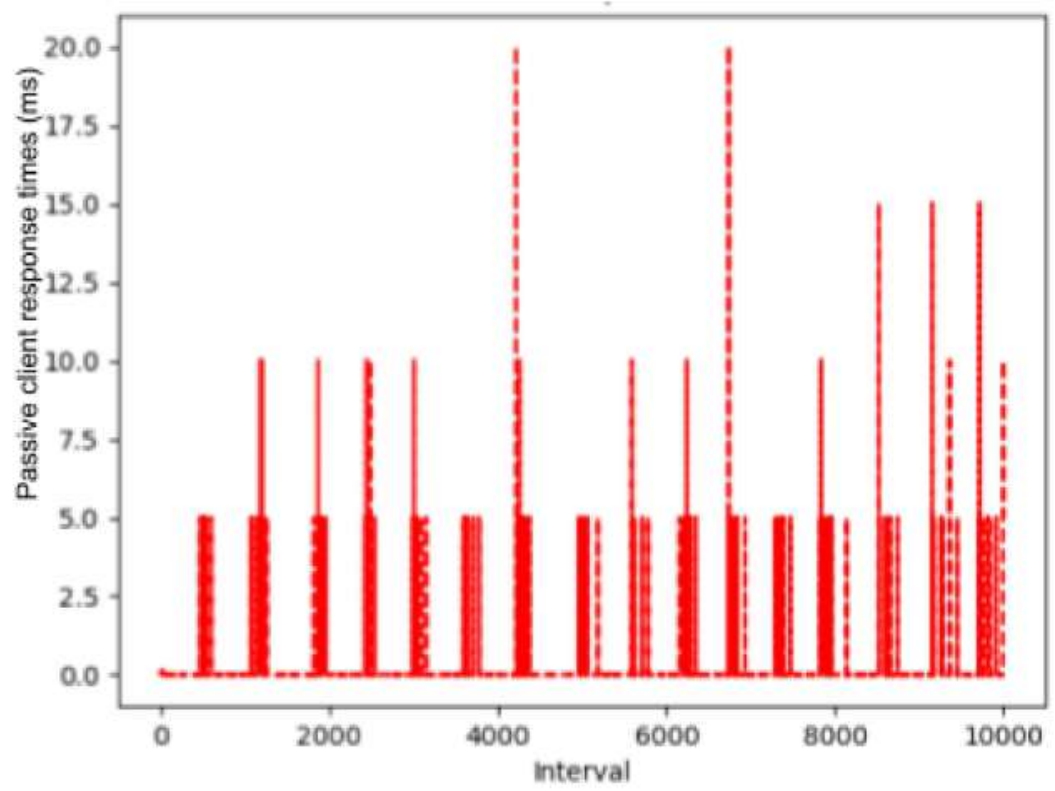

Figure 8.7.1.1: Passive Control Client Requests By Time Interval std $=0.734780534$, mean $=0.08251897, \max =20.017393, \min =0.003253$

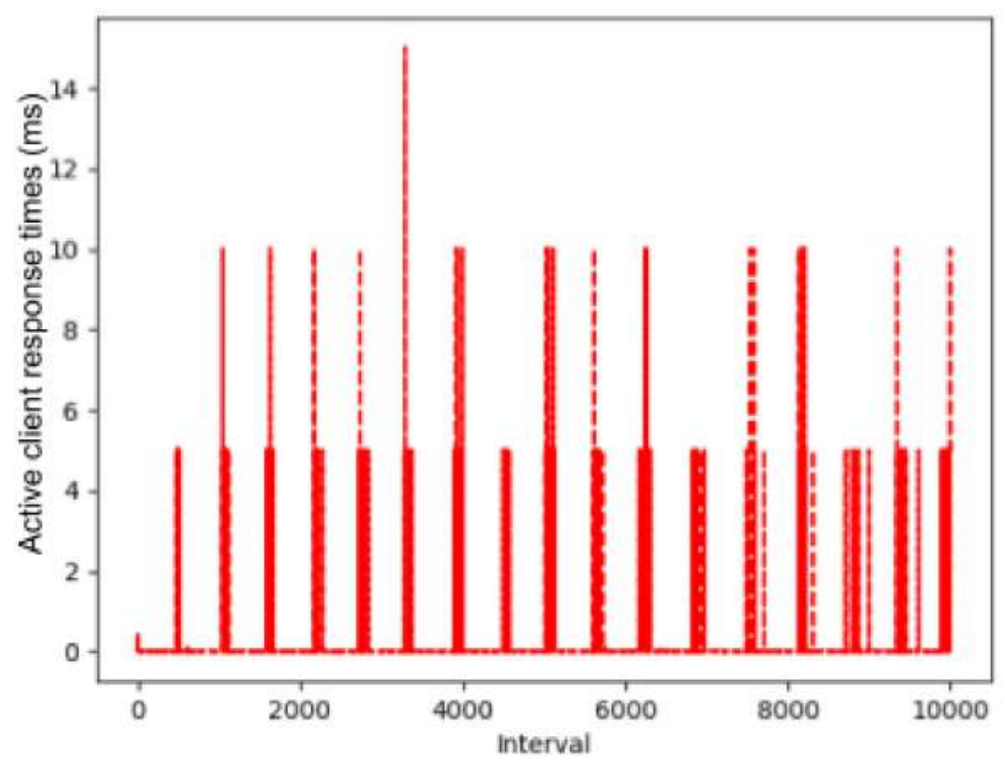

Figure 8.7.1.2: Active Control Client Requests By Time Interval 


$$
\text { std }=0.71539719, \text { mean }=0.08621096, \max =15.015683, \min =0.003292
$$

Figures 8.7.1.1 \& 8.7.1.2 show the impact of active control mode monitoring on the testbed client request time. One surprising outcome was that it led to a slightly higher average response time, raising the mean from $0.08252 \mathrm{~s}$ to $0.08621 \mathrm{~s}(4.472 \%$ change), probably due to random error in packet traversal times cross the network. A change of $3.69 \mathrm{~ms}$ is fairly within the range of network error (It is surprising because a reduction in response times is usually expected after provisioning control actions, however it is not guaranteed due to other factors like network delays caused by other devices running on the same cloud environment).

There was substantive improvement in the worst times (from a max of 20.02s to $15.02 \mathrm{~s}$ ) and a reduction in response time variance (standard deviation 0.7348 to 0.7154 ) due to elimination of other failure case response times. Multiple spikes between 10s and $15 \mathrm{~s}$ towards the end of the test run are eliminated in the active control mode data.

Performance metric values for the monitored testbed virtual machines are shown below. 
Normalized Process Load (\% CPU, Utilization, Memory) By Time

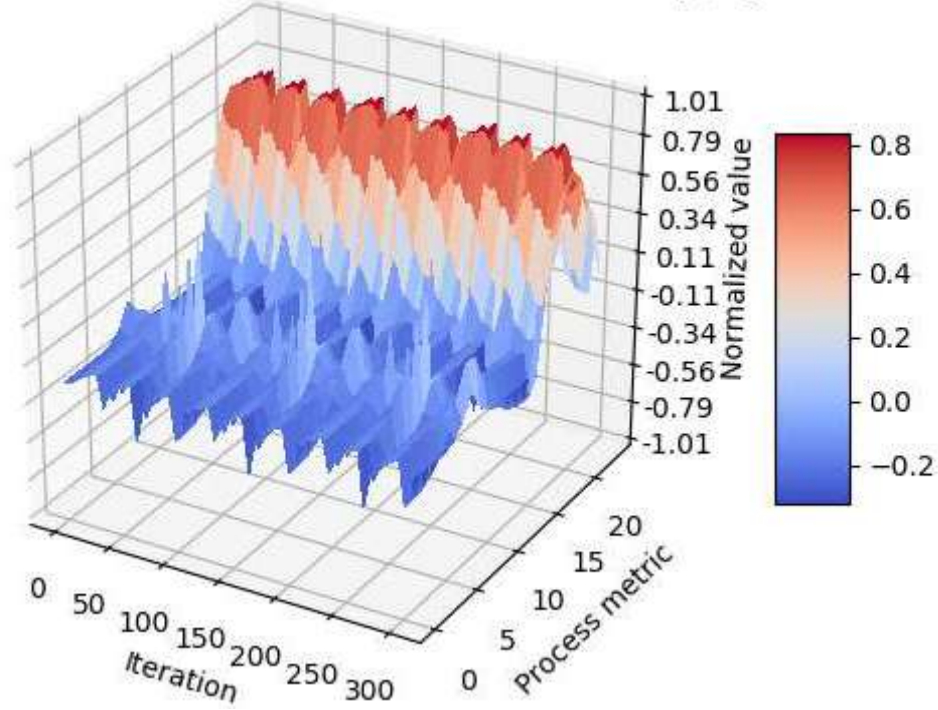

Figure 8.7.1.3: Passive monitor metrics for testbed application host

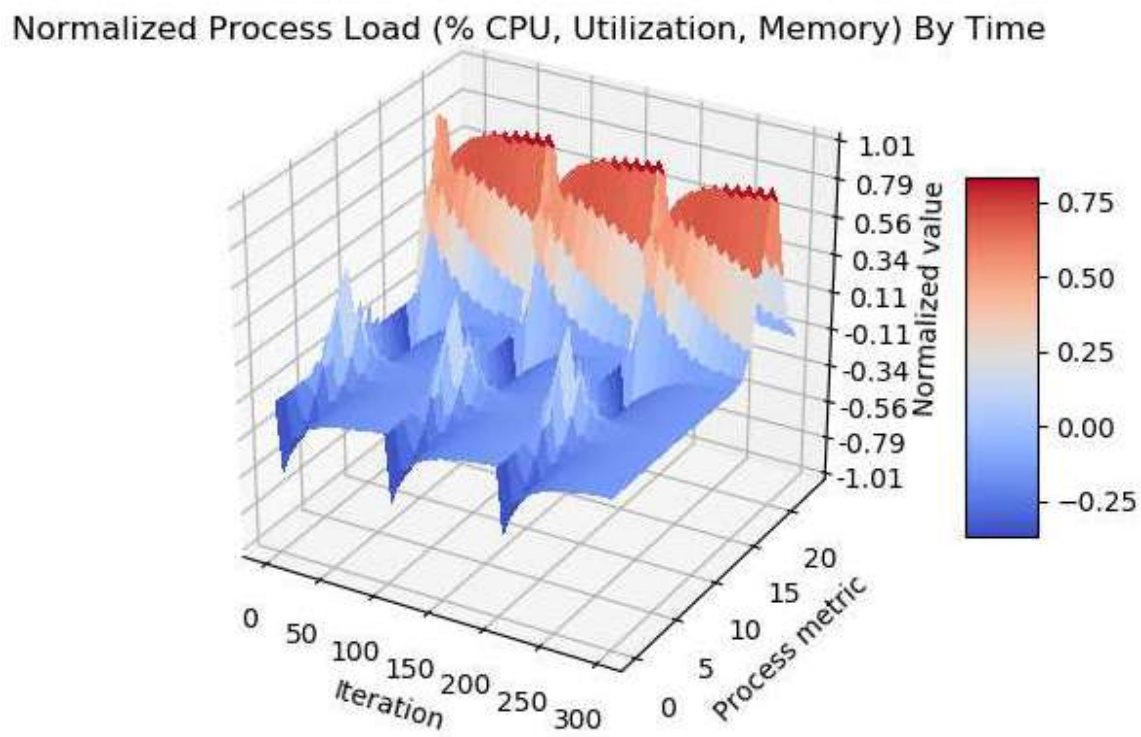

Figure 8.7.1.4: Active monitor metrics for testbed application host

Figures 8.7.1.3 \& 8.7.1.4 show the impact of active control mode monitoring on the application system. There metrics displayed there represent output from the linux 'top' command, tracked through time. There is a shift to lighter colours immediately after a 
control action (intervals $20,120 \& 220$ ), which is also indicated by a reduction in metric values, showing up as a trough in the above figures.

NB: The colours are more indicative of normalization differences per metric than they are time evolution of the metric.

\section{Active \& Passive Control Impact On Application Metric}

This section examines the end-to-end system, to evaluate the impact of control actions on the monitored application. The test environment is setup as in section 8.7. Overall there is a reduction in mean and standard deviation of testbed application CPU utilization for active control vis a vis passive control (Section 6.1.4.1).

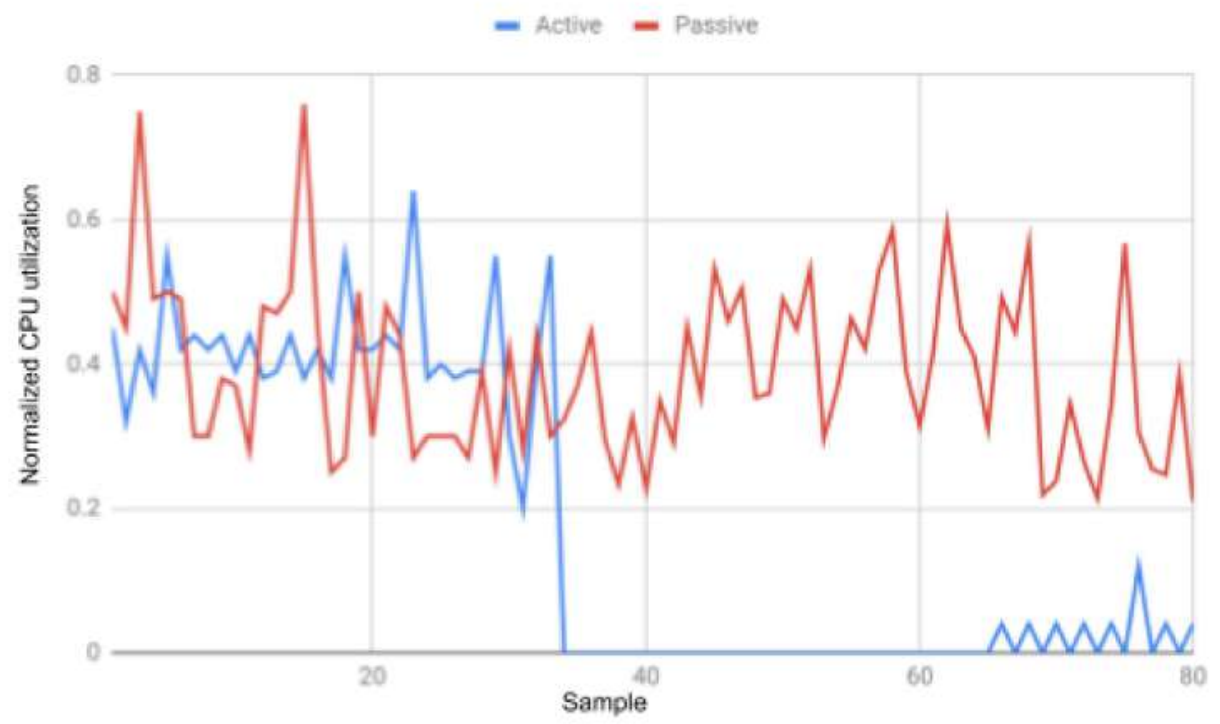

Figure 8.7.1.1.1: Active \& passive monitor impact on CPU utilization 
In summary, with active control there is a reduction in mean CPU usage from $41.96 \%$ to $17.83 \%$, as well as a corresponding increase in standard deviation (0.1228 to 0.2110$)$. The increase in overall standard deviation is slight, and the mean CPU usage in active control is outside the first standard deviation of the passive control CPU usage distribution.

There was substantive improvement in the client response worst times (from a max of $20.02 \mathrm{~s}$ to $15.02 \mathrm{~s}$ ) and a reduction in response time variance (standard deviation 0.7348 to $0.7154)$ due to elimination of other failure case response times. 


\section{Chapter 9: Controller Evaluation}

The controller uses learning-enabled model tracking to control a cloud application, one of the main goals of this project. This chapter evaluates the end-to-end system, with KF-LSTM-PCA for model estimation and single time step LQN predictions to drive control actions. Control actions involved executing configured commands (Section 6.1.5) to provision or deprovision resources required by the cloud application for request processing.

As per the experiment design chapter, the following evaluation results were compiled.

The goal is to provide a performance evaluation of the controller following recommended experiment guidelines [83].

The user-specified control actions are provided with a repetition factor called a burst size $(B R)$. This factor determines the strength of the provisioning and deprovisioning actions performed by the controller, and is a replication factor referring to the number of provisioning and deprovisioning commands issued during a single control action. The test scenarios include evaluating (all involve a single control action triggered as in section 6.1.5):

1. Predictive control for varying $B R$

2. Average predictive control over multiple iterations for varying BR

3. Average non-predictive control over multiple iterations for varying BR

4. Predictive control for single BR for various virtual machine types

5. Predictive control for varying BR for various virtual machine types 


\subsection{Evaluating Basic Predictive Control With Varying Burst Sizes}

This section evaluates the end-to-end system, with KF-LSTM-PCA for model estimation and LQN predictions to drive control actions, in a single experiment. Evaluation is done in terms of reduction in resource usage with control actions versus without them.

The controller is configured to function tracking the application metrics in predictive control mode (see section 6.1.4.1). The KF-PCA-LSTM unit's predictions drive the decision making logic of the controller. The workload includes continuous data retrieval client requests at a rate of 1 per ms as per section 7.3.

The goal is to evaluate the impact of control actions on resource usage by evaluating the impact of changes in the controller burst size (BR) on the application. Since BR indicates the number of control actions executed at a single time step, evaluating changing BR impact allows for a more detailed evaluation of the impact of control actions.

Overall there is slightly increasing drop off in usage for higher burst size as shown below:

\begin{tabular}{|l|l|l|l|}
\hline BR & 3 & 7 & 11 \\
\hline $\begin{array}{l}\text { CPU usage before } \\
\text { control action }\end{array}$ & 0.385 & 0.387 & 0.392 \\
\hline $\begin{array}{l}\text { CPU usage after } \\
\text { control action }\end{array}$ & 0.031 & 0.031 & 0.030 \\
\hline
\end{tabular}


Table 9.1: Change in CPU usage for various burst sizes

Below are a series of graphs showing the CPU time evolution under different burst sizes.

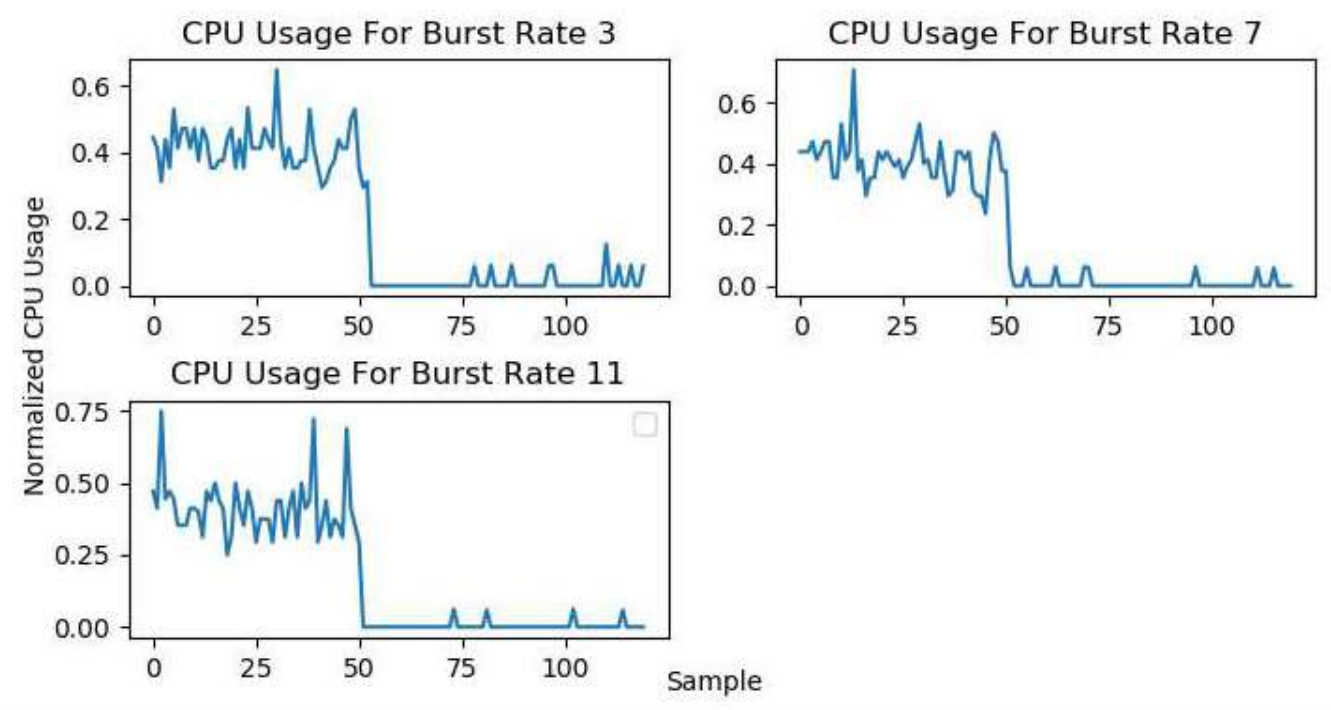

Figure 9.1.1: CPU Time for various burst sizes in predictive control

In each case the CPU usage drops from a mean of 0.39 to 0.03 after the controller

provisions more resources to the application, with increasing drop off for higher burst size $(0.385->0.031$ for $\mathrm{BR}=3,0.392->0.030$ for $\mathrm{BR}=11)$. 


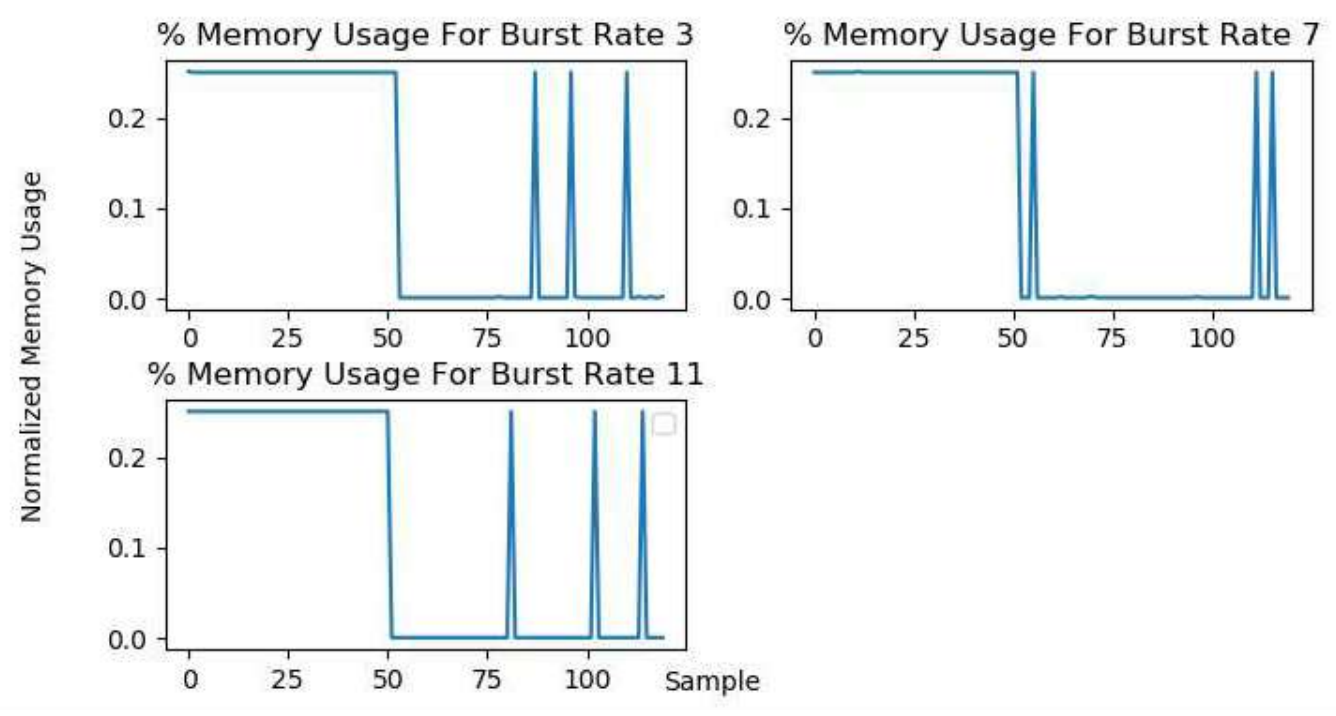

Figure 9.1.2: \% Memory usage for various burst sizes in predictive control

The memory usage percentage of the application has less variance than the CPU usage on average $(0.0138$ versus 0.0256$)$, and has a similar drop in value after controller provisioning actions issued in response to predicted high resource utilization, dropping from 0.2659 to 0.0175 with $\mathrm{BR}=3$.

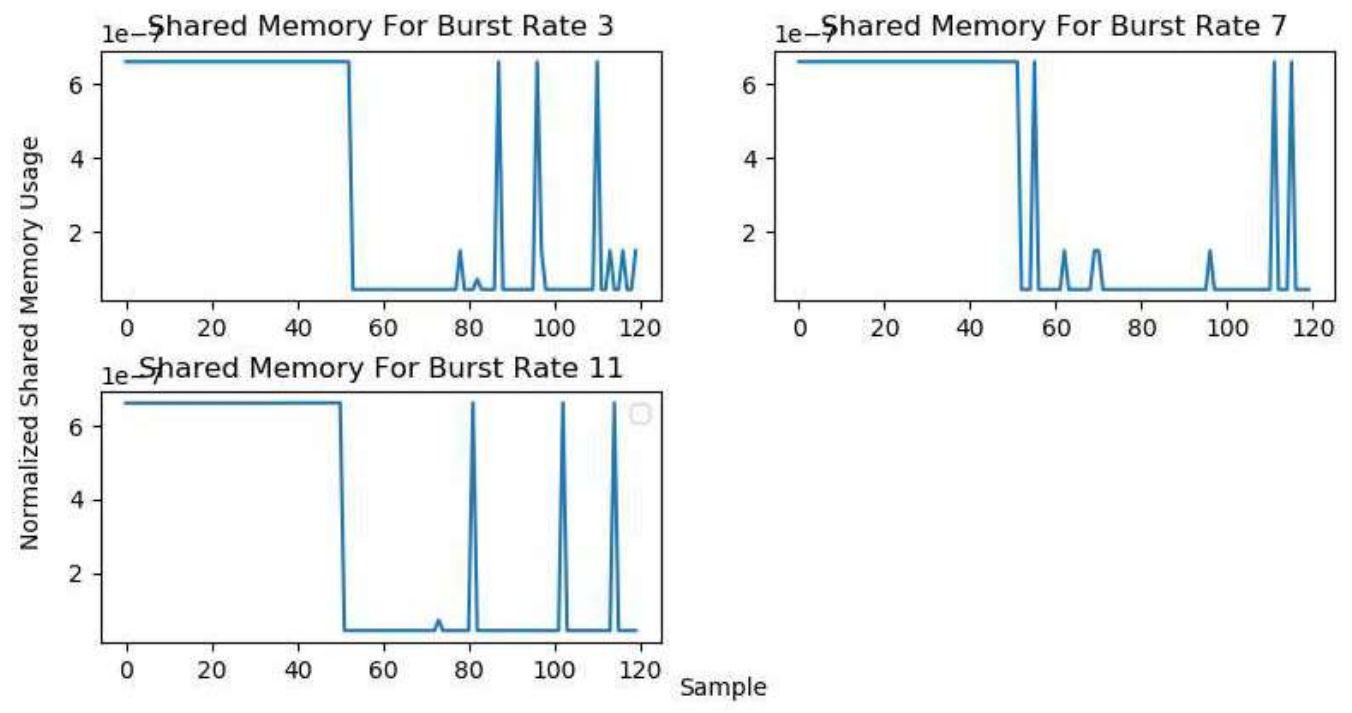

Figure 9.1.3: Shared memory for various burst sizes in predictive control 
The application's shared memory follows a similar trajectory to the overall memory percentage metric, with a bit more variance after provisioning actions. The spiking in shared memory after controller resource provisioning actions is similar to that seen when the system is at rest, probably due to context switching done by the operating system periodically. Observing the curves of \% memory and shared memory metrics, they seem to be more strongly correlated after the provisioning actions than the $\%$ memory is to the CPU usage (correlation drop of 19.10 versus 116.37 ).

\subsection{Evaluating Average Predictive Control With Varying Burst Sizes}

This section evaluates the end-to-end system, with KF-LSTM-PCA for model estimation and LQN predictions to drive control actions, in multiple experiments and aggregates results as outlined in this chapter's introduction.

This test scenario is similar to the basic test scenario above. However it is done over 10 experiments and evaluated as a statistical average over the experiments and the burst sizes. The workload includes continuous data retrieval client requests at a rate of 1 per ms as per section 7.3. The goal is to see how conserved behaviour is over multiple runs, with identical workload and initial conditions. Mean and standard deviations of resource values are calculated for each time step across 10 experiments. 

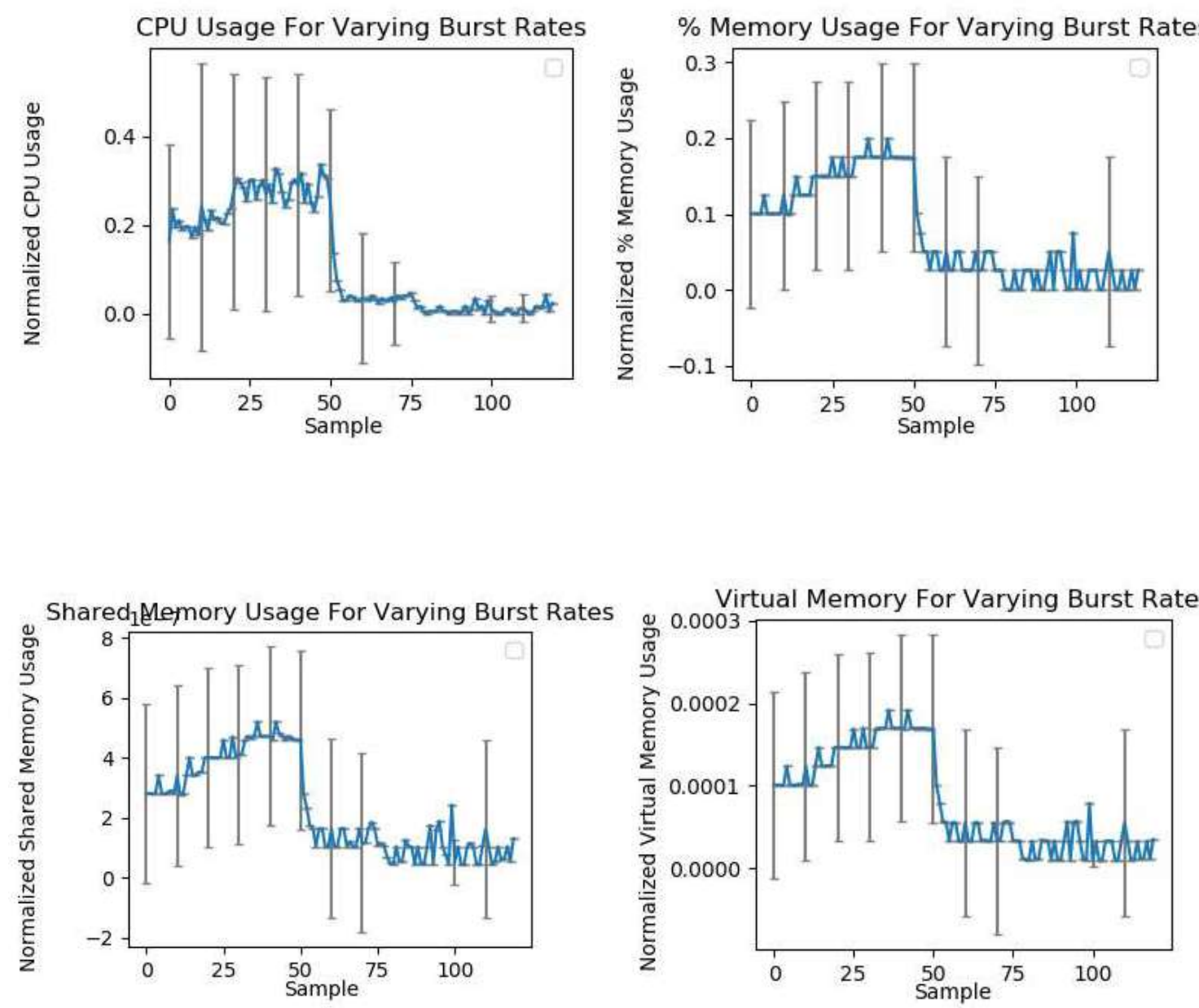

Figure 9.2.1: CPU, \% memory, shared \& virtual memory for varying burst sizes

On average over 10 iterations, the performance metrics have higher variance (compared to the single experiment test scenario) as is expected in measurements taken statistically from a non-deterministic system.

As is visible in the number of long error bars on the right hand side of the plots, the variance within each metric reduces after the controller provisioning actions. A drop in the metric values is also observed as expected. The reduction in variance is due to the reduced actual values (which approach zero after resource provisioning), which is an 
indicator of the stability of the reduction in application component utilization levels overall.
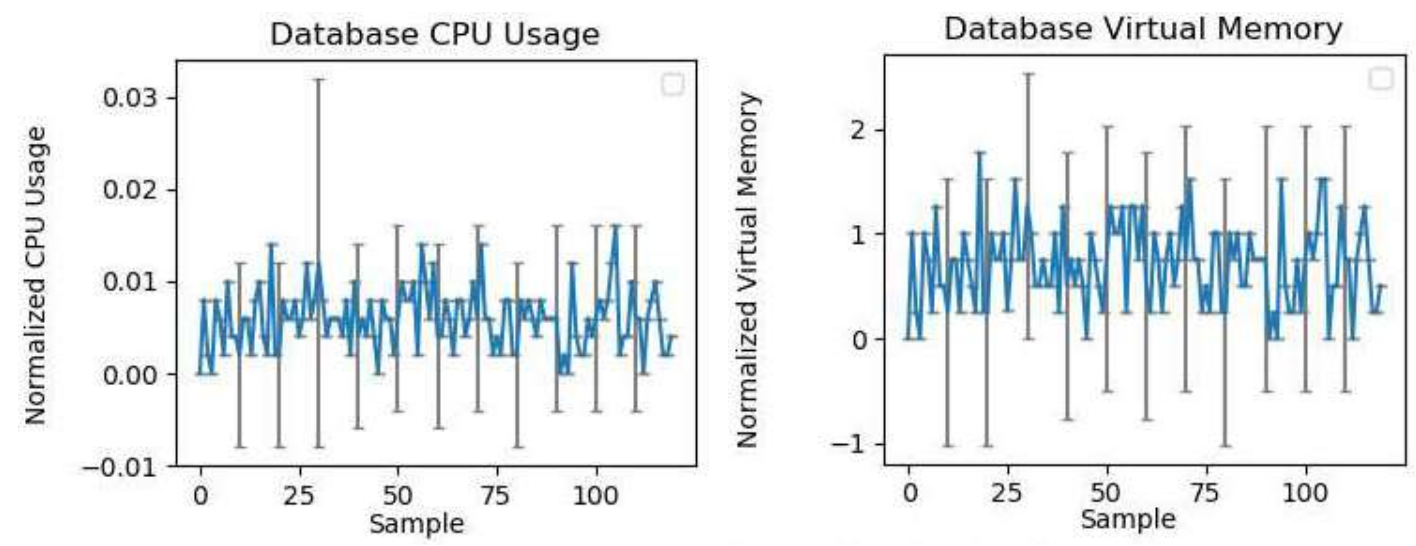

Figure 9.2.2: Shared memory for various burst sizes in predictive control

On the other hand, the database system showed little discernible change after resource provisioning. Note that the user-specified provisioning action was to increase the instances of the database and the search engine virtual machines in the application pool. The database system performance metric values are therefore expected to be less correlated to the provisioning action (triggered at around 50 samples - see previous figure). 


\section{Normalized Process Load (\% CPU, Utilization, Memory) By Time}

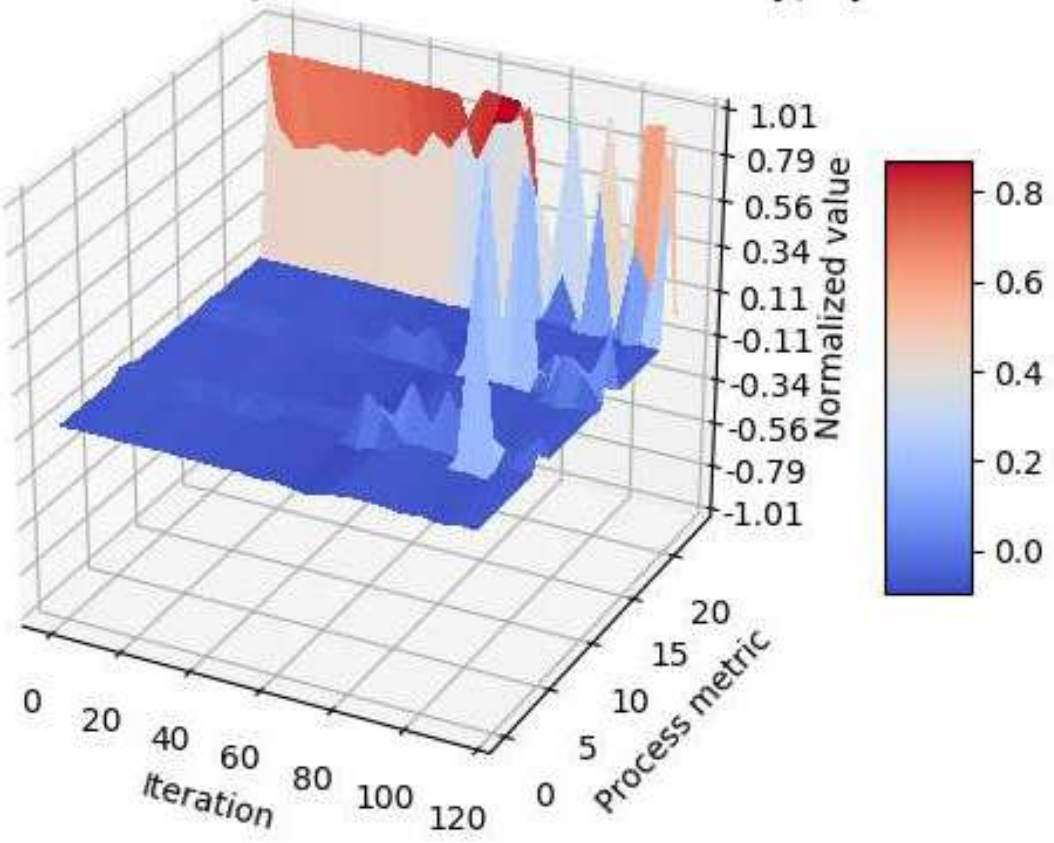

Figure 9.2.3: (24) Metrics for top 3 processes sorted by CPU usage over time

The resource provisioning action happens around iteration 50, after which the summary plot shows a reduction in the overall metric surface by the shift to lower value colours for the top process (process metrics 20 and up). The spikiness of the process metrics in this region is due to operating system time slices being handed to lower priority processes due to context switches when the top process becomes inactive.

\subsubsection{Note On Active Control Error Bars}

The error bars on each plot vary in size. These are real systems, and when multiple runs are made, the measured metrics will vary, even for an idle workload. Some of the error bars appear to be larger, evenly spaced at approximately ten sample intervals. These represent peaks and troughs in each run, determined by the response of the monitored 
application to the incoming workload. When the control actions are taken and resource utilizations drop in the right half of the plots, the roughly periodic large error bars reduce as expected (see figure 9.2.1) since the resource usage on each experiment reduce in magnitude after control actions.

\subsection{Evaluating Average Non-Predictive Control For Varying Burst Size}

This section runs the end-to-end system, with KF-LSTM-PCA for non-predictive model estimation and LQN calculations to drive control actions, in 10 experiments, and averages results. The purpose is to see the behaviour of the monitored application under non-predictive control, and make a comparison to predictive control.

This test scenario involves running a basic controller in non-predictive control mode (Section 6.1.4.1). It is configured to use the latest measure of performance metrics from the target application system, rather than predictions of the future performance metrics as provided by the tuned Kalman Filter.

However it is done over 10 experiments and evaluated as a statistical average over the experiments. The workload includes continuous data retrieval client requests at a rate of 1 per ms as per section 7.3. The goal is to see how conserved behaviour is over 10 runs, with identical workload and initial conditions. Mean and standard deviations of resource values are calculated for each time step across 10 experiments.

The expectation of this kind of system is that it would be slower at reacting to changes in the tracked system, and that will negatively impact the effectiveness of resource provisioning controller actions. 
The impact of controller driven resource provisioning actions is reduced with all the metrics showing a return to baseline levels $(0.3720$ mean for CPU usage and 0.2619 mean for $\%$ memory, 6.729 mean for shared memory and 0.00023 for virtual memory over intervals 1 to 20) within 30 sample intervals (between 21 and 50 samples approx.). This is likely due to the controller being slower to anticipate and react to the high usages, since it is in non-predictive control mode. Refer to Figure 9.2.1 for comparison to see how successful provisioning actions impact the monitored application.
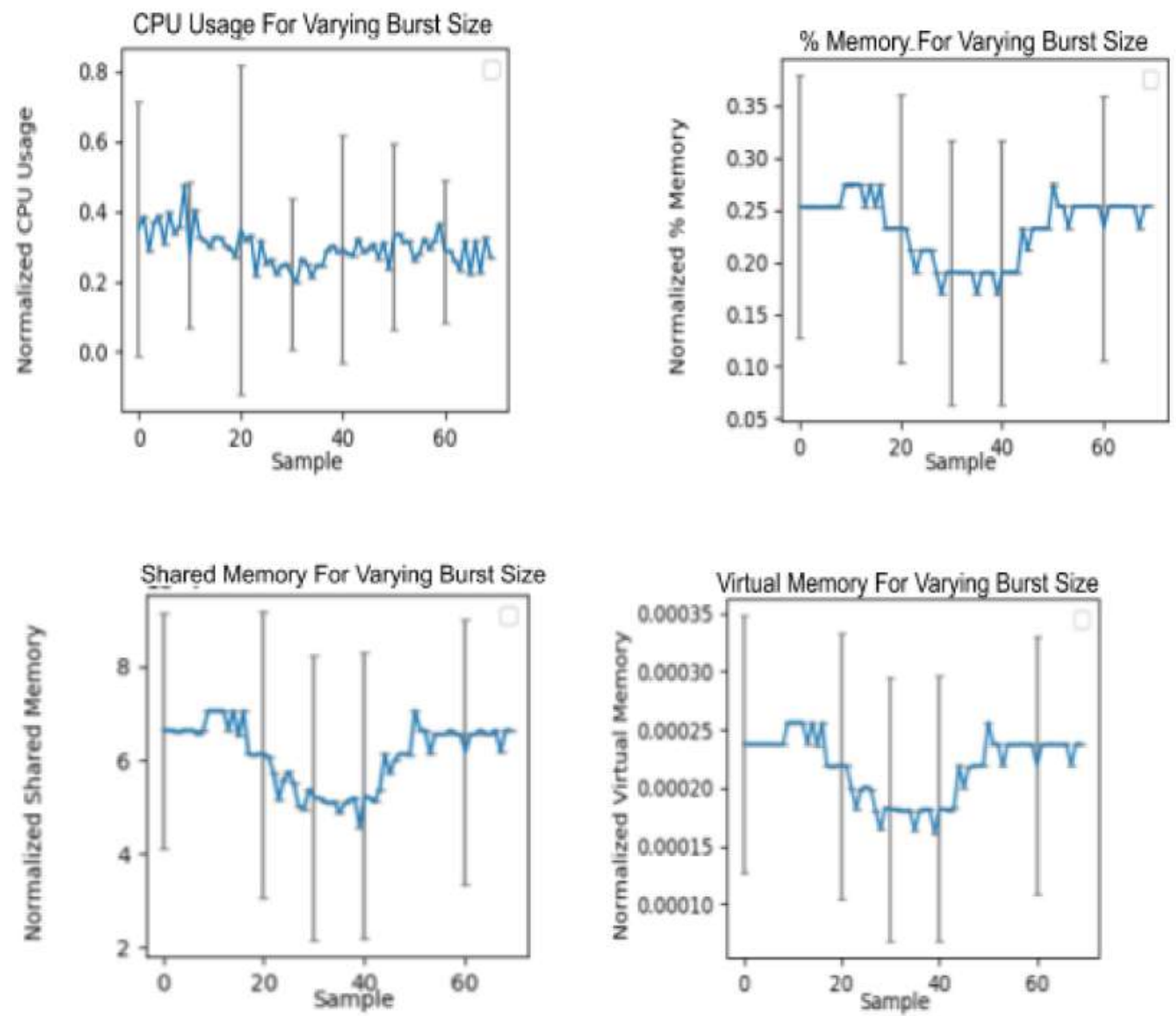

Figure 9.3.1: System metrics in basic mode for varying bust size

N.B: There are smaller error bars between the larger error bars in figure 9.3.1. 
The impact of controller driven resource provisioning actions is reduced with all the metrics showing a return to baseline levels $(0.3720$ mean for CPU usage and 0.2619 mean for $\%$ memory, 6.729 mean for shared memory and 0.00023 for virtual memory over intervals 1 to 20) within 20 sample intervals. The variance across runs, captured in the error bars, shows little change across resource provisioning. There is a slight increase in variance for shared and virtual memory metrics though not substantive enough to be of interest.

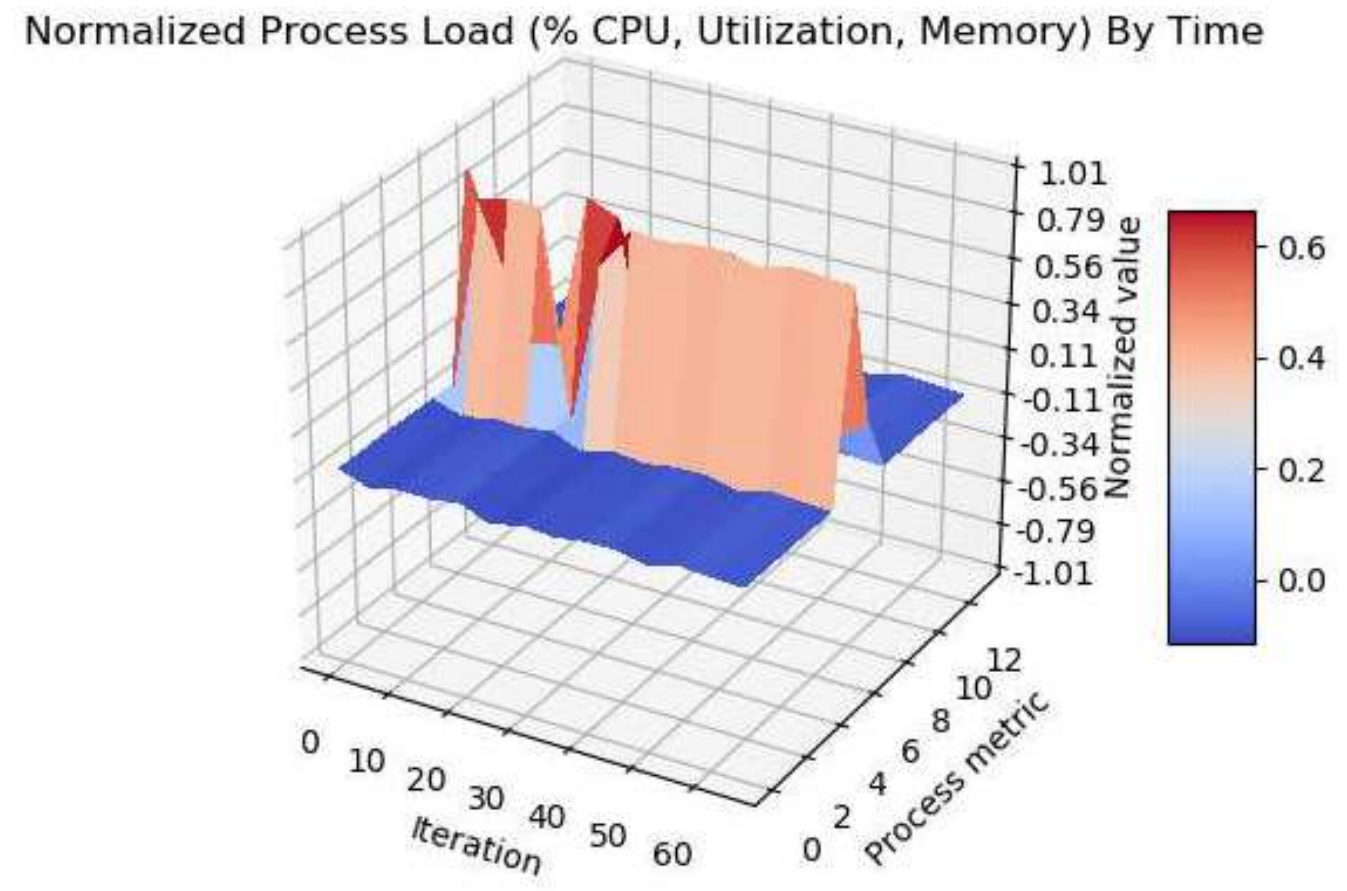

Figure 9.3.2: (24) Metrics for top 3 processes sorted by CPU usage over time Iin the individual metric plots above, the statistical average summary plot shows minor reduction in metric values around the provisioning action sample iteration, but otherwise no change beyond baseline values (the values in the first 20 intervals -0.3720 mean for 
CPU usage and 0.2619 mean for $\%$ memory, 6.729 mean for shared memory and 0.00023 for virtual memory) over the whole test period on average for all 10 test iterations. Figure 9.3.2 shows that most of the other metrics were not impacted much by application activity, and remained low as indicated by their blue color in the figure. Figure 9.2.1 shows how successful provisioning actions should impact the monitored application. There is an average normalized CPU usage over all samples of 0.3520 non-predictive control compared to 0.1683 for predictive control, a well over $109 \%$ improvement of this metric with predictive control.

\subsection{Evaluating Predictive Control By Burst Size For Virtual Machine Types}

This section evaluates the end-to-end system, with KF-LSTM-PCA for model estimation and LQN predictions to drive control actions, in a multiple experiments over a set of virtual machine types. The significance of this test is to see how the impact of the control actions and BR is affected by different virtual machine types.

Many of the virtual machine types (those with the suffix 'large') will be overprovisioned. The reason to have them is to see how the controller handles large application systems where resource thresholds are less likely to be crossed. It also allows for an investigation of whether the resources one has in mind regarding a large system are truly the resources that will improve application performance, or if counter-intuitive resource scarcity can still occur. The conclusion to this chapter does show that some counter-intuitive resource utilizations do occur. 
This test scenario involves using the predictive controller mode (section 6.1.4.1) to summarise application system metrics by virtual machine type. A set of virtual machines in Amazon Web Services were created from the same application instance image. A dedicated controller was also provisioned for each application virtual machine using a custom controller machine image. The controller is configured in predictive control mode (Section 6.1.4.1).

It is done over 10 experiments and evaluated as a statistical average over the experiments and the burst sizes. The workload includes continuous data retrieval client requests at a rate of 1 per ms as per section 7.3. The goal is to see how conserved behaviour is over 10 experiments, with identical workload and initial conditions. Mean and standard deviations of resource values are calculated for each time step across 10 experiments.

Virtual machine types are selected to provide a range of resource capacities from the small t2.micro type (1 vCPU and $1 \mathrm{GiB}$ memory) to the large m5.d12xlarge type (48 vCPU and 192GiB memory, $120 \mathrm{~Gb}$ network speed).

Table 9.4: Amazon Web Services EC2 Instance Types [84]

\begin{tabular}{|l|l|l|l|}
\hline VM Type & \# of vCPU & Memory $(\mathrm{GiB})$ & Network $(\mathrm{Gb})$ \\
\hline c4.large & 2 & 3.75 & moderate \\
\hline c5.24xlarge & 96 & 192 & 25 \\
\hline i3.en24xlarge & 96 & 768 & 100 \\
\hline i3.large & 2 & 15.25 & 10 \\
\hline m4.large & 2 & 8 & Moderate $(<5)$ \\
\hline m5.d4xlarge & 16 & 64 & 10 \\
\hline
\end{tabular}




\begin{tabular}{|l|l|l|l|}
\hline m5.d12xlarge & 48 & 192 & 10 \\
\hline m5.large & 2 & 8 & 10 \\
\hline
\end{tabular}

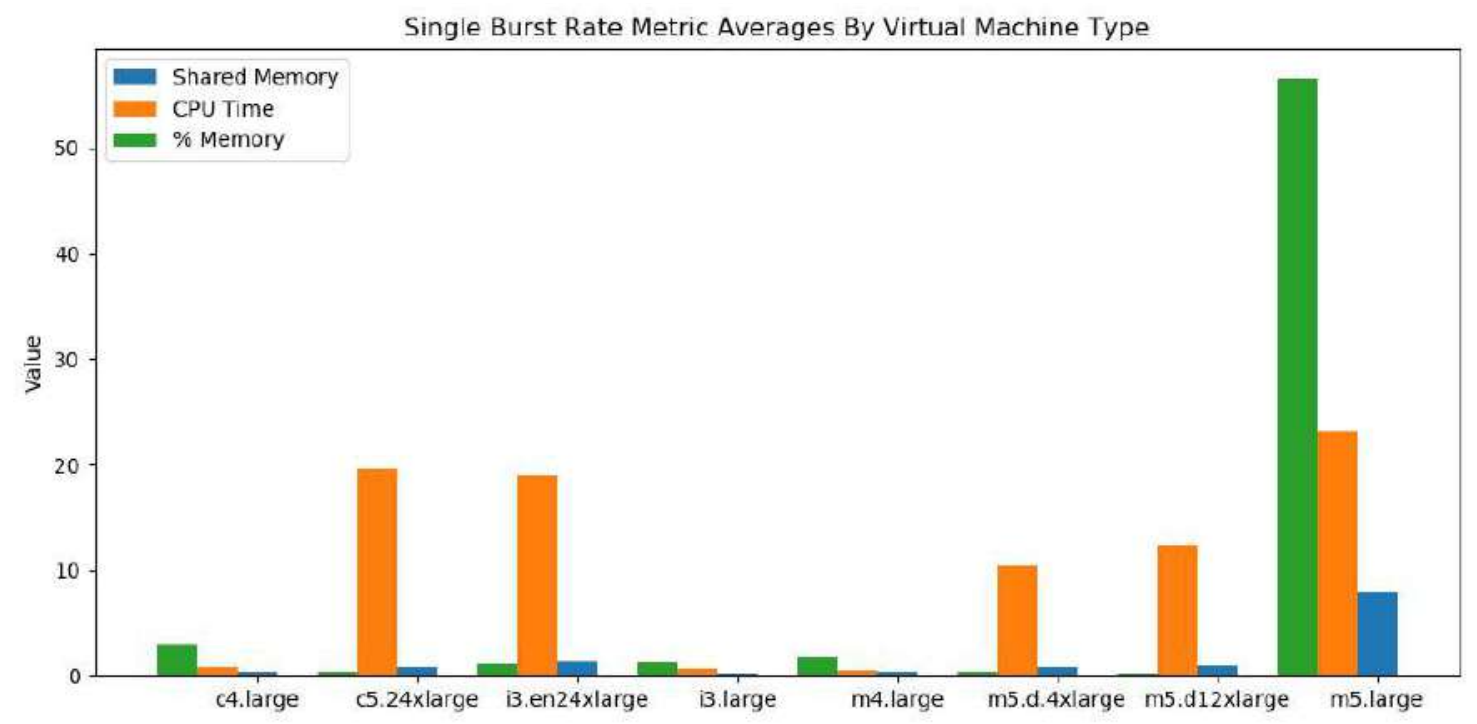

Figure 9.4.0.1: CPU time, \% memory and shared memory by virtual machine type

The m5.large type has an Elastic Book Store (EBS) bandwidth of 2120 Mbps while the m4.large type has an EBS bandwidth of only $450 \mathrm{Mbps}$.

Now, the overall client traffic and application runtime should be expected to load the smaller virtual machines with lower capacities (like the c4.large and m4.large) more than the higher capacity virtual machines.

However, the impact of the controller resource provisioning actions will vary depending on the normalized level of utilization of the application system metric.

1. There is a trend towards higher average CPU usage on the larger machines (c5.24xlarge - 20\%, i3.en24xlarge - 19\%), lower usage on the midsize machines 
(m5.d4xlarge - 10\%, m5.d12xlarge - 13\%) and lowest usage on the small machines (c4.large, i3.large, m4.large all $<1 \%$ ). This is because there was less controller-driven resource provisioning activity on these machines as they did not cross user-specified utilization thresholds due to their higher capacity.

2. The m5.large machine (23\% CPU usage) did not cross the user-specified resource thresholds during the iterative tests. It therefore did not follow the trend expected in 1) above, and is an example of the limitation of using a trigger-based control algorithm, especially when the thresholds are user specified.

Single Burst Rate Metric Averages By Virtual Machine Type

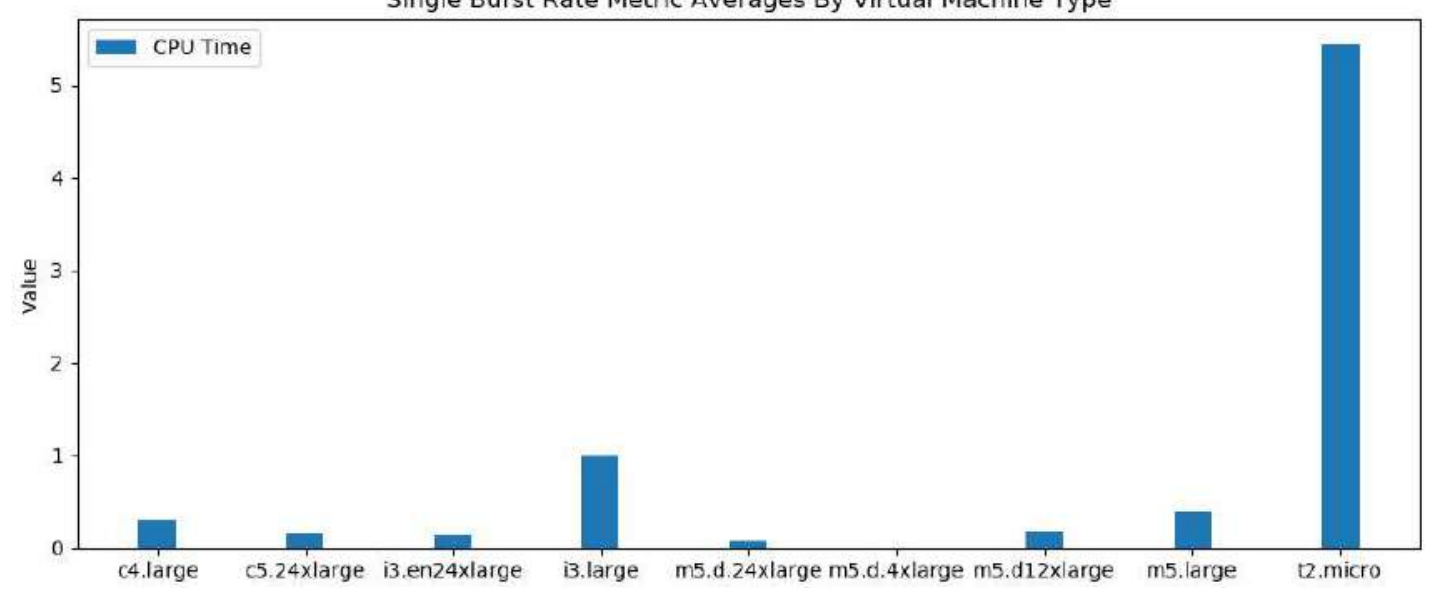

Figure 9.4.0.2: CPU time by virtual machine type (minus $m 4$.large for readability)

\subsubsection{Predictive Control For Virtual Machine Types At Multiple Burst Sizes}

An analysis of metric values by virtual machine type at different burst sizes is shown below. The test scenario involved running client traffic on each application virtual 
machine instance each with a dedicated controller virtual machine. The workload includes continuous data retrieval client requests at a rate of 1 per ms as per section 7.3. Predictive control actions (section 6.1.5.1) are produced automatically, configured at various burst sizes. These steps are then repeated over multiple iterations (10) and averaged for analysis.

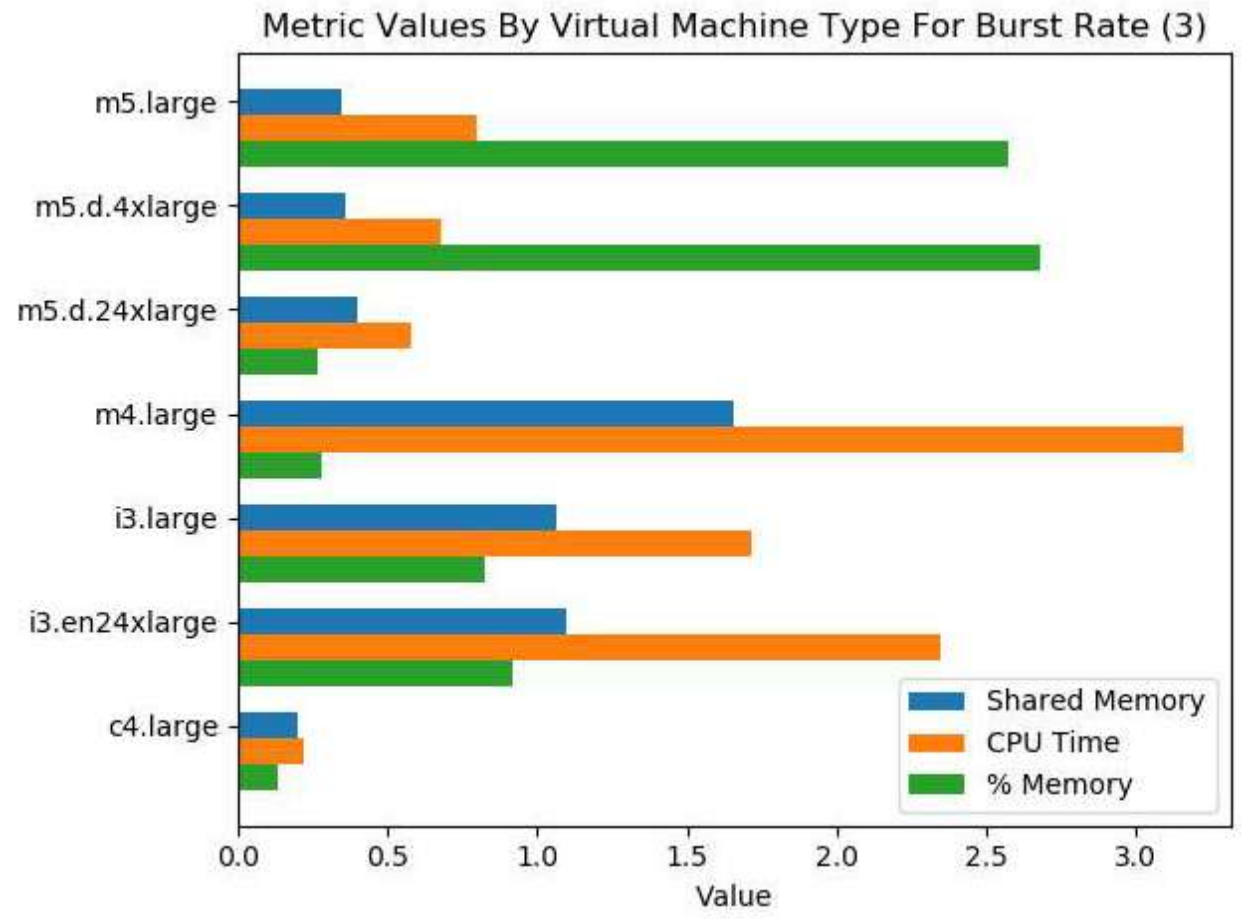

Figure 9.4.1: Metric Values By VM Type For Burst Size (3)

Similar to the test scenario in the non-predictive controller (see section 6.1.4.1), the low burst size of 3 had a pattern of increasing normalized metric values from lower tier 
(c4.large, i3.large) to mid tier (m5.d4xlarge, m5.d24xlarge) to higher tier (i3.en24xlarge).

The outlier again is m4.large type, which is an outlier for the same basic reason as explained in the previous section, namely that it did not cross the user-specified trigger threshold.

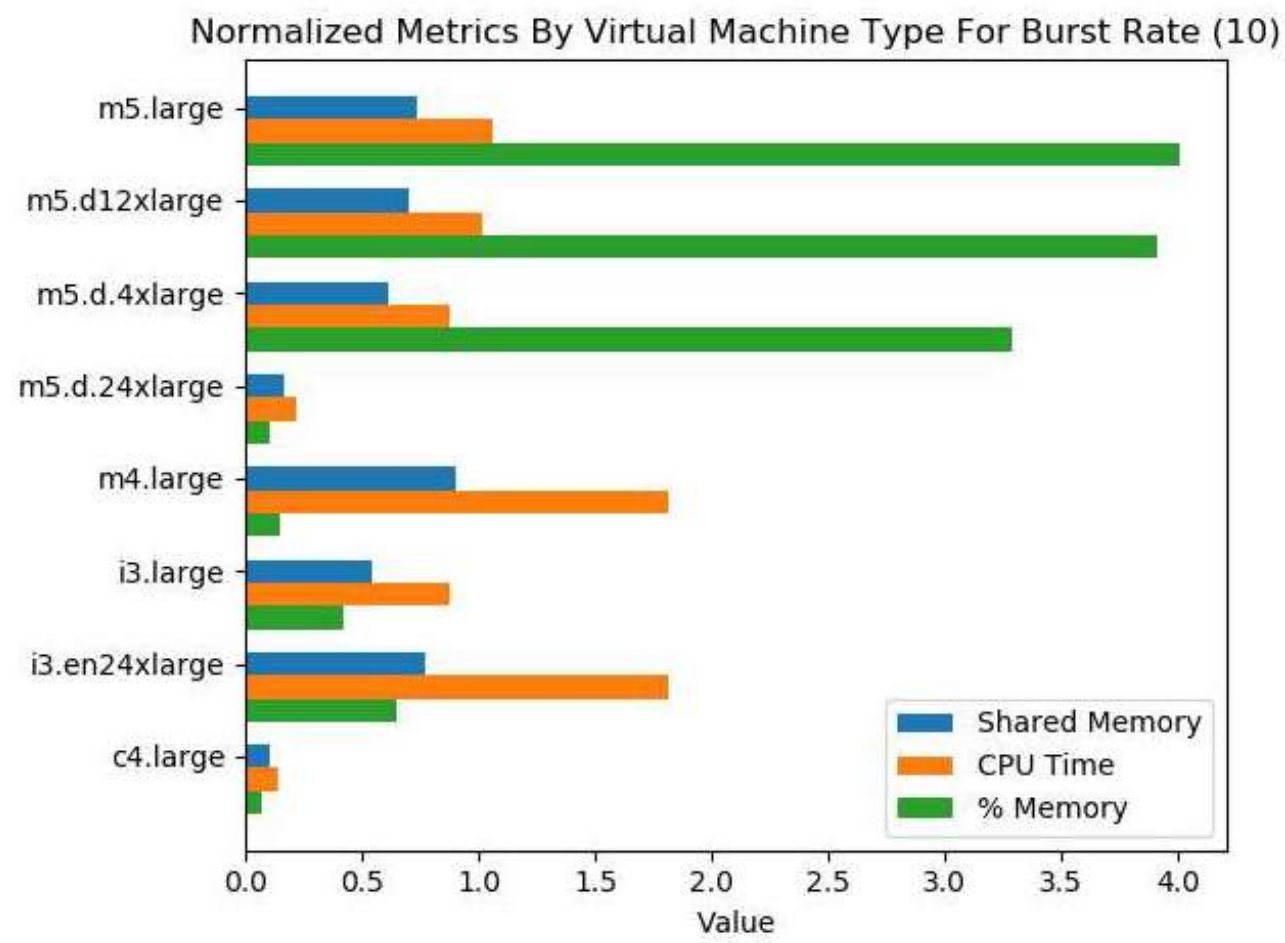

Figure 9.4.2: Normalized Metric Values By VM Type For Burst Size (10)

In this scenario a similar pattern is seen in terms of CPU (and overall) usage levels, and virtual machine instance type. However there is an inversion regarding \% memory usage on the mid-tier virtual machines, which have an unusually high value approaching $400 \%$. This is probably due to high amounts of kernel switching as the operating system moves between process and threads. Investigating the specific details seems out of the scope of 
this analysis, suffice it to say the low tier virtual machines had less context switching demands due to fewer resource provisioning actions, and high tier virtual machines had more memory available hence did not reach as high a percentage of utilization.

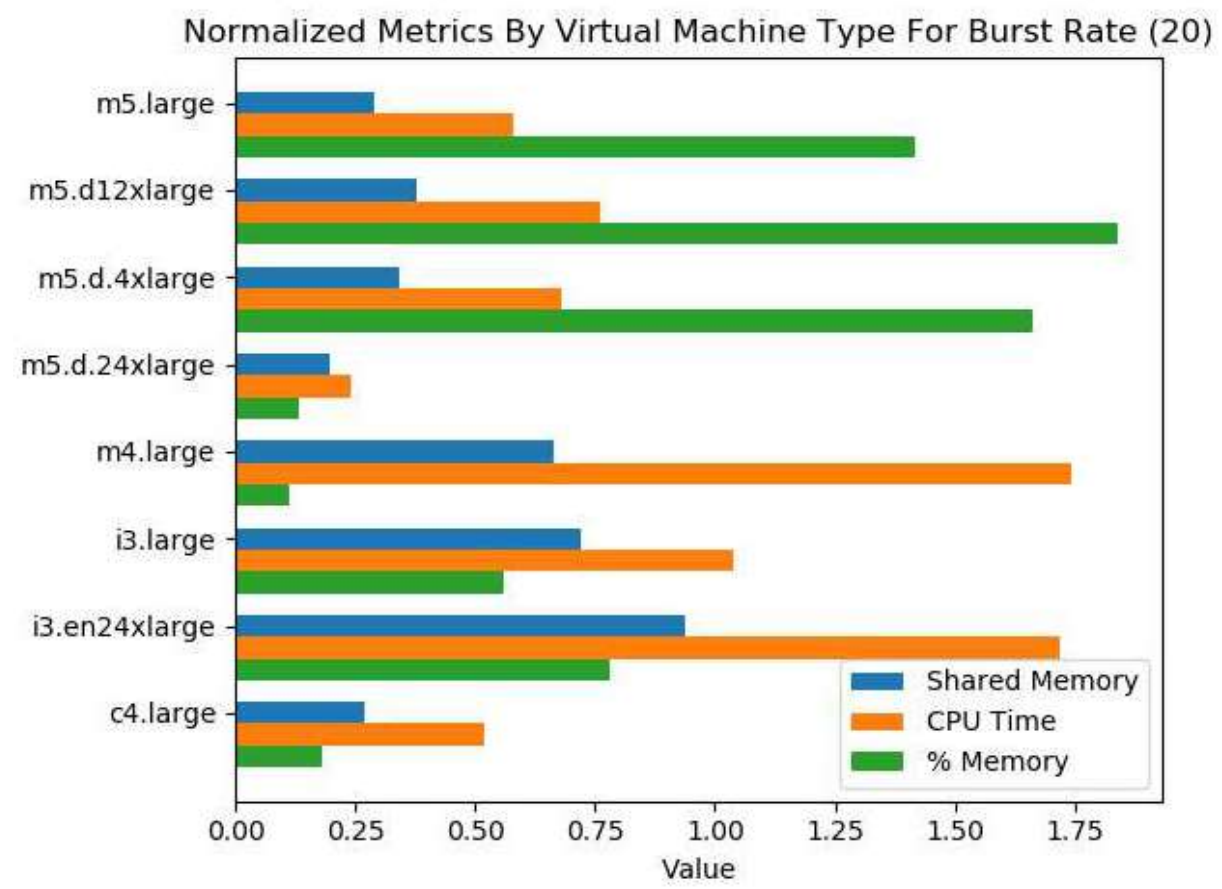

Figure 9.4.3: Normalized Metric Values By Virtual Machine Type For Bust Rate (20)

In this test scenario, the same pattern is seen regarding CPU usage. However, \% memory usage on the mid-tier virtual machine types reduces to the $150-175 \%$ range. A higher burst size would lead to a more robust application resource allocation, leading to reduced kernel switching on the application machine instances.

Selection of user-specified model-based thresholds is critical, and has to be matched appropriately to the size and type of the application virtual machine to be monitored. Basic thresholds did work in approximately $90 \%$ of the cases in this evaluation, a 
multi-type analysis like this is useful in customizing a threshold set specific to each target application and cloud setup.

CPU Usage For Various Burst Sizes Sorted By VM Capacity (low to high)

\begin{tabular}{|l|l|r|l|r|}
\hline VM Type & BR=1 & BR=3 & BR=10 & BR=20 \\
\hline c4.large & 0.9418 & 0.2619 & 0.241 & 0.5219 \\
\hline m4.large & 0.519 & 3.28 & 3.275 & 1.7613 \\
\hline m5.large & 24.33 & 0.7295 & 0.793 & 0.6318 \\
\hline i3.large & 0.2751 & 1.739 & 1.807 & 1.102 \\
\hline m5.d4xlarge & 11.71 & 0.7366 & 0.729 & 0.7387 \\
\hline m5.d12xlarge & 13.46 & 0.6821 & 0.738 & 0.7831 \\
\hline c5.24xlarge & 20.1 & $\mathrm{xxx}$ & 0.2749 & 0.2964 \\
\hline i3.en24xlarge & 19.62 & 2.397 & 2.416 & 1.739 \\
\hline
\end{tabular}




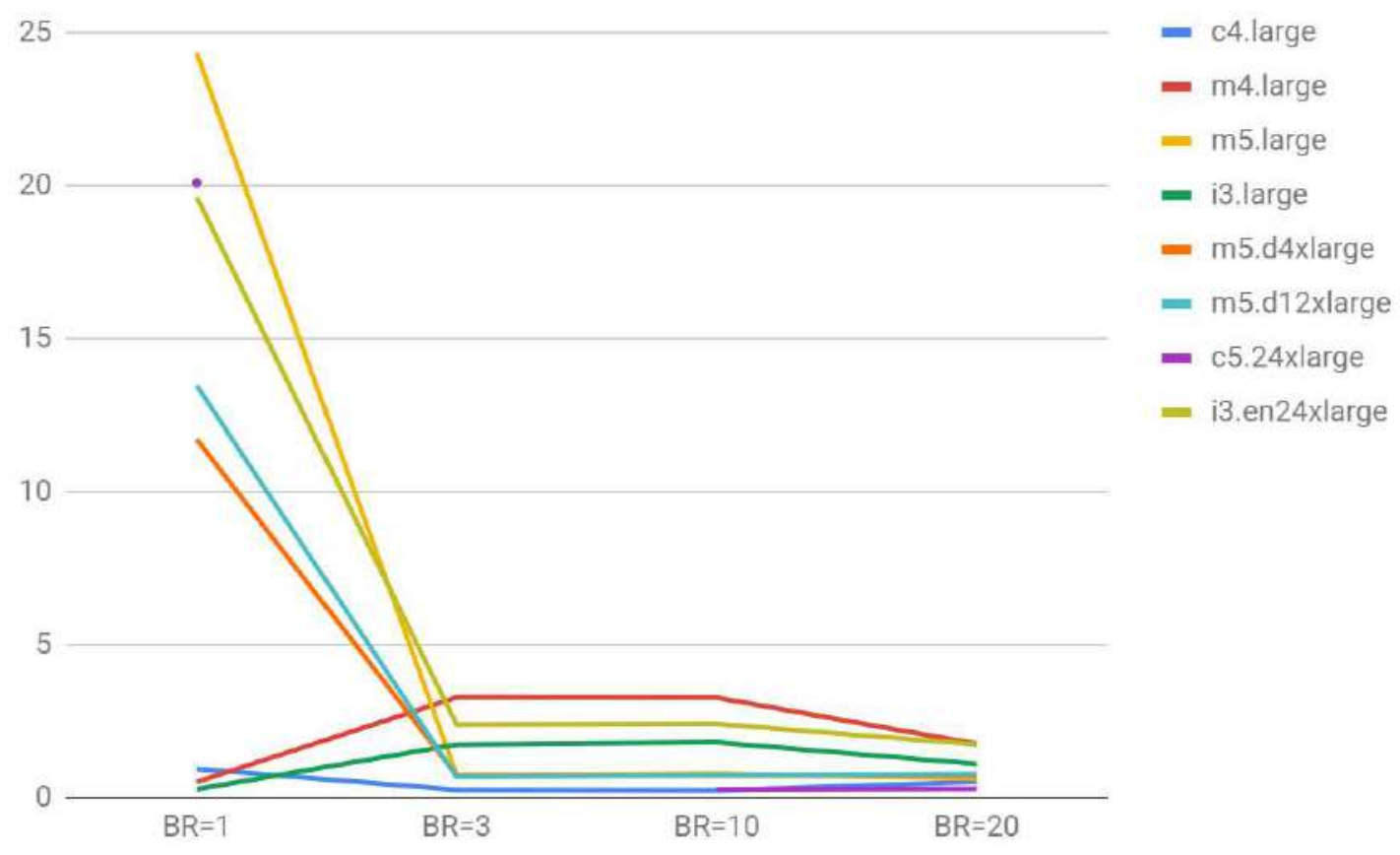

Figure 9.4.4: CPU Usage For Increasing Burst Rates

Further supporting the fact that increasing burst size reduces resource usage on controlled virtual machines is the above plot. There are some observations:

1. The overall trend is for resource usage to reduce as burst size increases

2. The large machines have relatively higher resource usage for low burst sizes (BR $=1$ ). It supports the observation on section 9.4 that provisioning control actions with low burst rates have much lower resource utilization impact on the large virtual machines. In order to see significant resource usage reductions, increase in BR is necessary (BR $>=3$ in this analysis), and this is dependant on the virtual machine setup in question, and is to be determined on a case-by-case basis. It 
could be the case that the 'larger' machines could have more numerous but slower CPUs, hence the higher CPU usage for low burst rates.

3. An analysis of this type is a good way to determine the critical controller configuration in terms of determining optimal BR (3) for the selected user-specified utilization thresholds (default of $10 \%$ for all metrics in this test) for the controller and target system.

Overall it can be seen that the provisioning control actions have a significant impact on resource utilization on controlled applications, such as is shown in Table 9.1, Figure 9.3.3, the figure above (Figure 9.4.1) and others in this chapter. Test applications have shown orders of magnitude reductions in resource usage (e.g. CPU usage reduced from $38.5 \%$ to $3.1 \%$ for $\mathrm{BR}=1$ in Table 9.1), although this is totally dependent on the test application environment. 


\section{Chapter 10: Conclusion}

Going back to the contribution regarding the impact of LSTM tuning on Kalman filter performance, there are improvements in Kalman filter performance as shown in section 8.3 (in both KF-LSTM1 \& KF-LSTM2 algorithms). Overfitting is mitigated by using randomized input features in KF-LSTM, and by online active learning (using training samples) across different time periods. In summary, for signals with exponential derivatives, tuning has substantial gains in tracking accuracy (increase in mean accuracy $\Delta \mu=29 \%$ and reduction in standard deviation, $\Delta \sigma=-0.356)$. Tracking sinusiod signals also shows small gains in accuracy after tuning ( $\Delta \mu=0.79 \%$ and $\Delta \sigma=-0.00214)$. As shown in section 8.4 , for the proposed active learning algorithm, there is observed a bit of fluctuation in Kalman Filter accuracy over the first LSTM training epochs which stabilizes, eventually exceeding $90 \%$ after sufficient LSTM training epochs. Also, there is a tendency of the training data acquisition process to get stuck at suboptimal coefficients. To avoid these 'local minima', randomized coefficients are inserted.

Proposing a control algorithm using model-driven actions to monitor and control an application in real time (Section 6.1.4.2) was another key contribution. This allowed for the prediction of application metrics using KF-LSTM1 (Section 8.4) and the ability to reason logic about and select optimal control actions. The control algorithm runs in each monitor thread, allowing for scalable application control over a cloud of control system hosts. 
The (single virtual machine type) control algorithm testbed analysis covers the impact of the control algorithm on the monitored application. One surprising outcome was a higher average client response time, raising the mean from $0.08252 \mathrm{~s}$ to $0.08621 \mathrm{~s}$ ( $4.472 \%$ change), probably due to random error in packet traversal times cross the network. A change of $3.69 \mathrm{~ms}$ is fairly within the range of network error. There was substantive improvement in the worst case client response times (from a max of 20.02s to 15.02s) and a reduction in response time variance (standard deviation 0.7348 to 0.7154 ) due to elimination of other failure case response times.

Regarding the contribution of implementing an adaptive controller using LSTM tuning of Kalman filters (Section 6.1) and evaluate performance gains, various lessons were learned. In the cloud system analysis in chapter 9, all virtual machine types had controller driven performance gains that were very sensitive to user specified thresholds. In general if the thresholds (which are percentages) are the same for small and large virtual machine controllers, the larger virtual machines are less likely to cross thresholds for the same application and workload. Hence more nuanced threshold specification is needed, tailored to the machine type, as is shown in detail in section 9.4. In the cloud system analysis it was seen that ad hoc user-specified trigger thresholds worked in around $90 \%$ of the cases in terms of enabling the controller to reduce overall resource usage. However for a guarantee of proper controller-driven resource optimization, a targeted cloud system analysis is essential in determining optimal user-specified trigger thresholds (section 9.4.1). 
A key observation about the system environment is the level of activity during tuning compared to that during operation (section 8.1). If the tuning environment is not representative of the operating environment, such that there are fewer active processes, or the system load is lower, or there are more resources available, then the tuned Kalman Filter will be less accurate. A representative system load, and rich mix of active processes is key for effective tuning to occur. Also, if there is a dramatic increase in process activity towards the end of the tuning cycle, this information might not get incorporated into the model, hence prolonging tuning past this 'burst' in activity would be advisable.

For the purpose of exploring model tracking and proposing a scheme for estimating model parameters of applications in real time, the KF-PCA algorithm is a key contribution (Chapter 4). As shown in section 8.6 the combination of PCA inference and Kalman filter prediction had an impact on error rates, with variance in absolute error $10.72 \%$ for the plain Kalman filter and $0.5467 \%$ for the combined approach (section 8.6.1). Where as the PCA-based approach shows robustness to noise, it does show dropout errors when it does encounter noise, hence dropping the least significant eigenvectors to shift to the most significant subspace (dimensionality reduction [98]) helps reduce noise sensitivity. Essentially the PCA-Kalman approach is more accurate because there are two steps of noise reduction (eigenvector subspace projection and KF), when compared to a pure Kalman filter. 


\subsection{Threats to Validity}

The use of the cloud for experiments is a non-ideal environment as cloud performance data can be influenced by hidden factors. On the other hand the cloud is the intended application area for the controller, so there is a clear advantage to doing the controller experiments and analysis in the cloud vis a vis having a real world analysis of the controller.

There is potential for a more complete analysis. However, this is only a feasibility study, hence:

- A comparison with additional competing approaches to machine learning and modeling was not done

- Constraints in time and cost of experiments precluded a full statistical analysis of the results (e.g, confidence intervals for all measures)

\subsection{Future Enhancements \& Research Topics}

There are various areas that can be explored to expand this analysis. Some of the basic ones include looking at customizing weights and biases and learning rates to characterize their effects on noise coefficient matrix discovery. Exploring methods for determining a good learning rate would be beneficial, such as cyclical rating, where varying learning rates between epochs linearly or exponentially is done in order to determine the optimal values [11]. 
For computing systems that have set workload patterns or lower levels of activity variation, recurrent neural networks can also likely be used for the predictive aspects of the controller. Exploring these in isolation, or in tandem with Kalman Filters would be of interest in comparing and contrasting performance with the system under consideration here. Parameter determination techniques like Maximum Likelihood or Bayesian estimation [12] could also be worth exploring regarding Kalman Filter noise coefficients. It would be of interest to augment the feature set used to train the LSTM to see if greater prediction accuracy can be realized. Also, Kalman Filters do predictions for the next time step. It would be useful to be able to extend the prediction horizon further into the future so as to avoid unnecessary provisioning done due to short term metric fluctuations. The active monitor implemented here used a single tuning phase followed by an operational phase for the Kalman Filter. It would be of benefit to update it in future versions to periodically retune the LSTM \& EKF units. This periodic tuning will continue through the life cycle of the controller so as to adapt to drift and configuration changes in the system under control, as well as the cloud environment in general. 


\section{Appendices}

\section{Appendix A : Background on the Extended Kalman Filter (EKF)}

The Kalman Filter is usually used as an estimator of the state vector of the linear model of a changing system. In cases where the model is nonlinear, the Extended Kalman Filter was developed [8].

The basic idea of the EKF is to linearize the state-space model at each time interval where the next state and measurement update are calculated as shown below:

$x_{k+1}=f\left(k, x_{k}\right)+w_{k}$

$y_{k+1}=h\left(k, x_{k}\right)+v_{k}$

where $\mathrm{w}_{\mathrm{k}}$ and $\mathrm{v}_{\mathrm{k}}$ are independent, zero mean, white gaussian noise processes with $\mathrm{R}_{\mathrm{k}}$ and $\mathrm{Q}_{\mathrm{k}}$ vectors as outlined in earlier sections. We then define

$\mathrm{F}_{\mathrm{k}+1, \mathrm{k}}=\frac{\delta f(k, x)}{\delta x} \mid \mathrm{x}=\mathrm{x}_{\mathrm{k}}$

$\mathrm{H}_{\mathrm{k}}=\frac{\delta h(k, x)}{\delta x} \mid \mathrm{x}=\mathrm{x}_{\mathrm{k}}^{-}$

with $\mathrm{H}_{\mathrm{k}}$ \& $\mathrm{F}_{\mathrm{k}}$ being linear approximations of the nonlinear $\mathrm{f}\left(\mathrm{k}, \mathrm{x}_{\mathrm{k}}\right)$ and $\mathrm{h}\left(\mathrm{k}, \mathrm{x}_{\mathrm{k}}\right)$

Once the approximations are found, typically as first order derivatives of the nonlinear model functions from the previous time step, standard Kalman Filter equations are applied.

Continuing now as in a standard Kalman Filter, for $\mathrm{k}=0$ we have

$\widehat{x}_{0}=\mathrm{E}\left[\mathrm{x}_{0}\right]$ 
$\mathrm{P}_{0}=\mathrm{E}\left[\left(\mathrm{x}_{0}-\mathrm{E}\left[\mathrm{x}_{0}\right]\right)\left(\mathrm{x}_{0}-\mathrm{E}\left[\mathrm{x}_{0}\right]\right)^{\mathrm{T}}\right]$

and for $\mathrm{k}=1,2 \ldots$

$\widehat{x}_{\mathrm{k}}{ }^{\mathrm{f}-\mathrm{f}}=\mathrm{F}_{\mathrm{k}, \mathrm{k}-\mathrm{x}} \widehat{x}_{\mathrm{k}-1}^{\mathrm{f}-}$

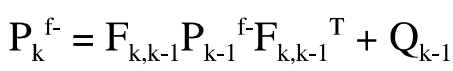

$\mathrm{G}_{\mathrm{k}}^{\mathrm{f}}=\mathrm{P}_{\mathrm{k}-1}{ }^{\mathrm{f}}{ }^{-} \mathrm{H}_{\mathrm{k}}{ }^{\mathrm{T}}\left[\mathrm{H}_{\mathrm{k}} \mathrm{P}_{\mathrm{k}} \mathrm{H}_{\mathrm{k}}{ }^{\mathrm{T}}+\mathrm{R}_{\mathrm{k}}\right]^{-1}$

$\widehat{x}_{\mathrm{k}}{ }^{\mathrm{f}}=\widehat{x}_{\mathrm{k}}{ }^{\mathrm{f}-\mathrm{f}}+\mathrm{G}_{\mathrm{k}}{ }_{\mathrm{k}}^{\mathrm{f}}\left(\mathrm{y}_{\mathrm{k}}{ }^{\mathrm{f}}-\mathrm{H}_{\mathrm{k}} \widehat{x}_{\mathrm{k}}{ }^{\mathrm{f}-}\right)$

These are the state estimation, error covariance propagation, Kalman gain estimate and state update [8].

\section{A.1 EKF Prediction-based Controller Actions}

The controller uses the state predictions from the Kalman Filter to drive logic for optimization of system resources. This creates an adaptable, robust controller able to anticipate system loads and make efficient accommodations. 


\section{A.2 EKF Observation and Model Summary}

For detailed background on the internal state of the Extended Kalman Filter during operation (prediction, update phases), please see reference [5].

Testing operation of the EKF module [2] used in this project is covered below. Various known input signals were tracked by the Extended Kalman filter, and the output is shown below. Note that in all cases there is a time lag before the EKF is sufficiently updated and starts changing trajectory to approach.

A key feature of Kalman Filters is that as recurrent estimators history has no determination on the next prediction of the EKF, only the state estimate from the prior time step and the current measurement are needed.
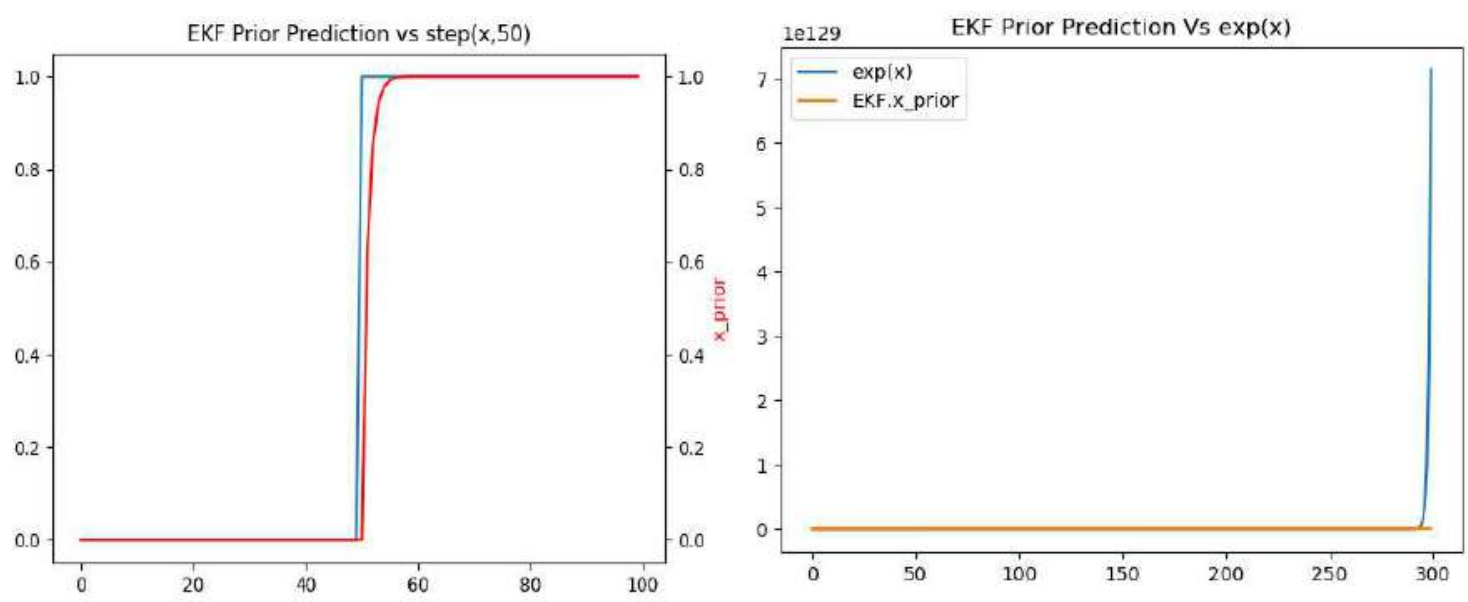

Figure A.2.0: EKF tracking for $\mathrm{z}_{\mathrm{k}}=\left\{\begin{array}{ll}0 & \text { if } x<50 \\ 1 & \text { if } x \geq 50\end{array}\right.$ and $\mathrm{z}_{\mathrm{k}}=\exp (\mathrm{k})$ 

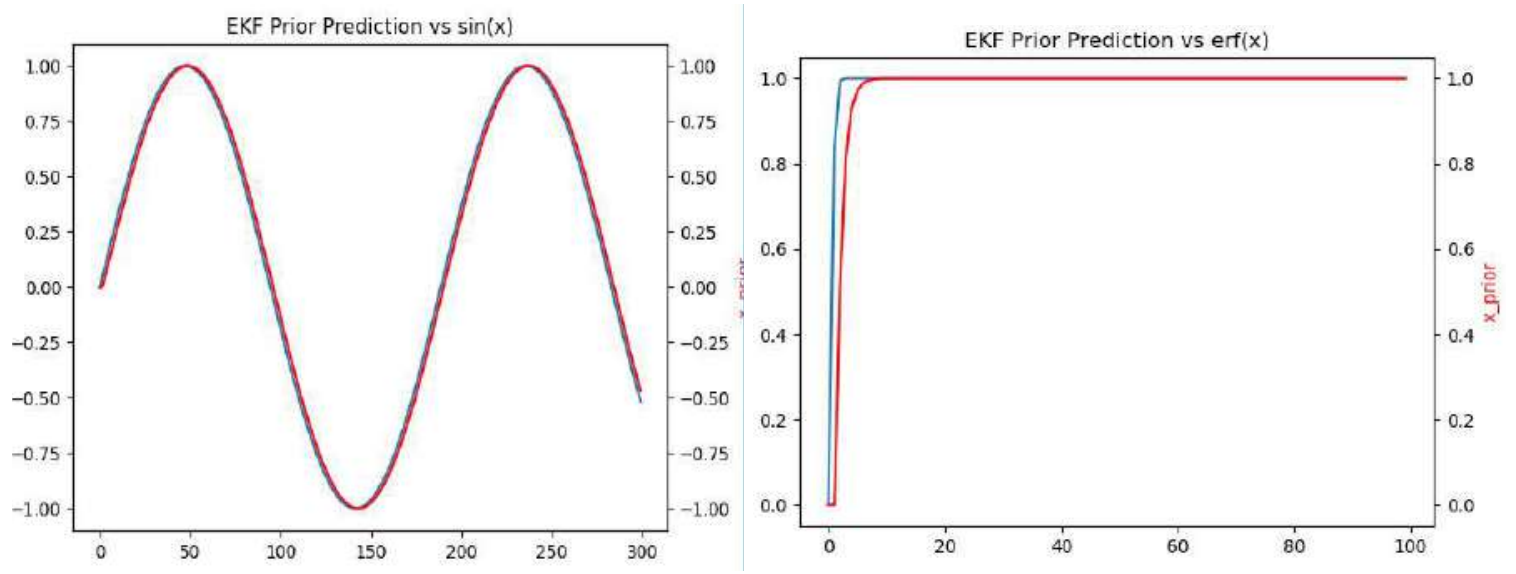

Figure A.2.1: EKF testing for $\mathrm{z}_{\mathrm{k}}=\sin (\mathrm{k})$ and $\mathrm{z}_{\mathrm{k}}=\operatorname{erf}(\mathrm{k})$

In all the unit tests the default values for $\mathrm{Q}, \mathrm{R}, \mathrm{F}$ and $\mathrm{H}_{\mathrm{k}}$ matrix, in the case of the Extended Kalman Filter Python library, are all the identity matrix. In the case of the state transition matrix this leads to good predictions for signals with gradients near identity, but lots of prediction errors for signals with gradients that diverge from identity. A good example of this is Figure 8.3.0.1 where the signal has an effective slope of 1.01 (which is only slightly above 1) times identity. The tracking of a tuned EKF is consistently better than the default EKF, and the default EKF's errors accumulate over time for signals of that type, degrading performance significantly if the filter is not tuned correctly. 


\section{Appendix B Appendix B: Long Short Term Memory (LSTM) Implementation}

The LSTM unit's initialization, training and testing steps are outlined below in detail.

They are basically standard procedures adopted from

\section{B.1 LSTM Initialization}

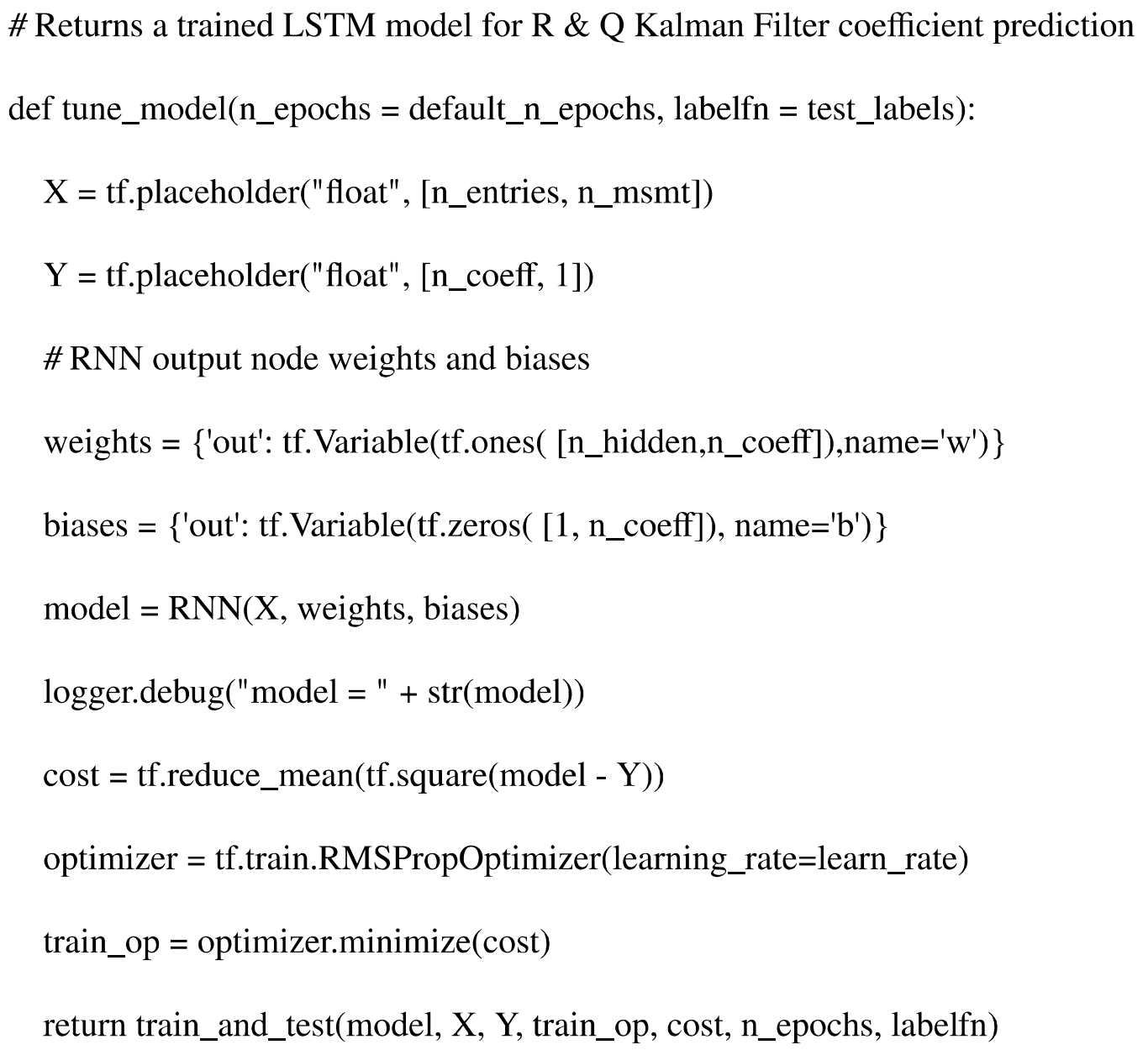




\section{B.2 Training \& Testing}

\# Train and Test the LSTM model using a custom labeling function

def train_and_test(model, X, Y, train_op, cost, n_epochs, labelfn=test_labels):

test_data $=[]$

\# Training

for epoch in range $\left(0, \mathrm{n} \_\right.$epochs $)$:

batch_data = labelfn $($ model, $\mathrm{X})$

train_data $=$ batch_data $[0:$ int $($ len $($ batch_data $) * 0.75)]$

test_data $=$ test_data + batch_data $[\operatorname{int}(\operatorname{len}($ batch_data $) * 0.75):]$

for (i, (batch_x, batch_y)) in zip(range(len(train_data)), train_data):

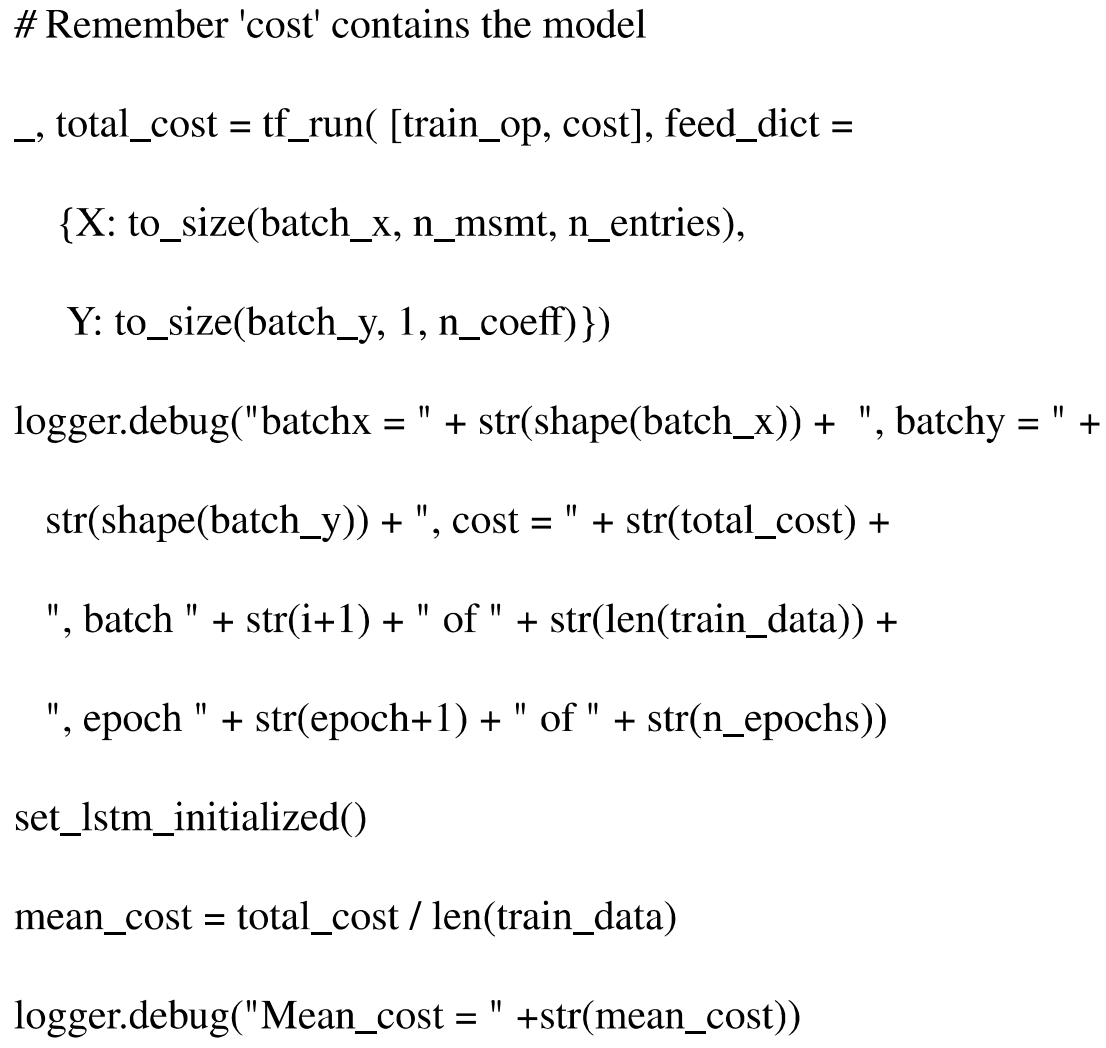




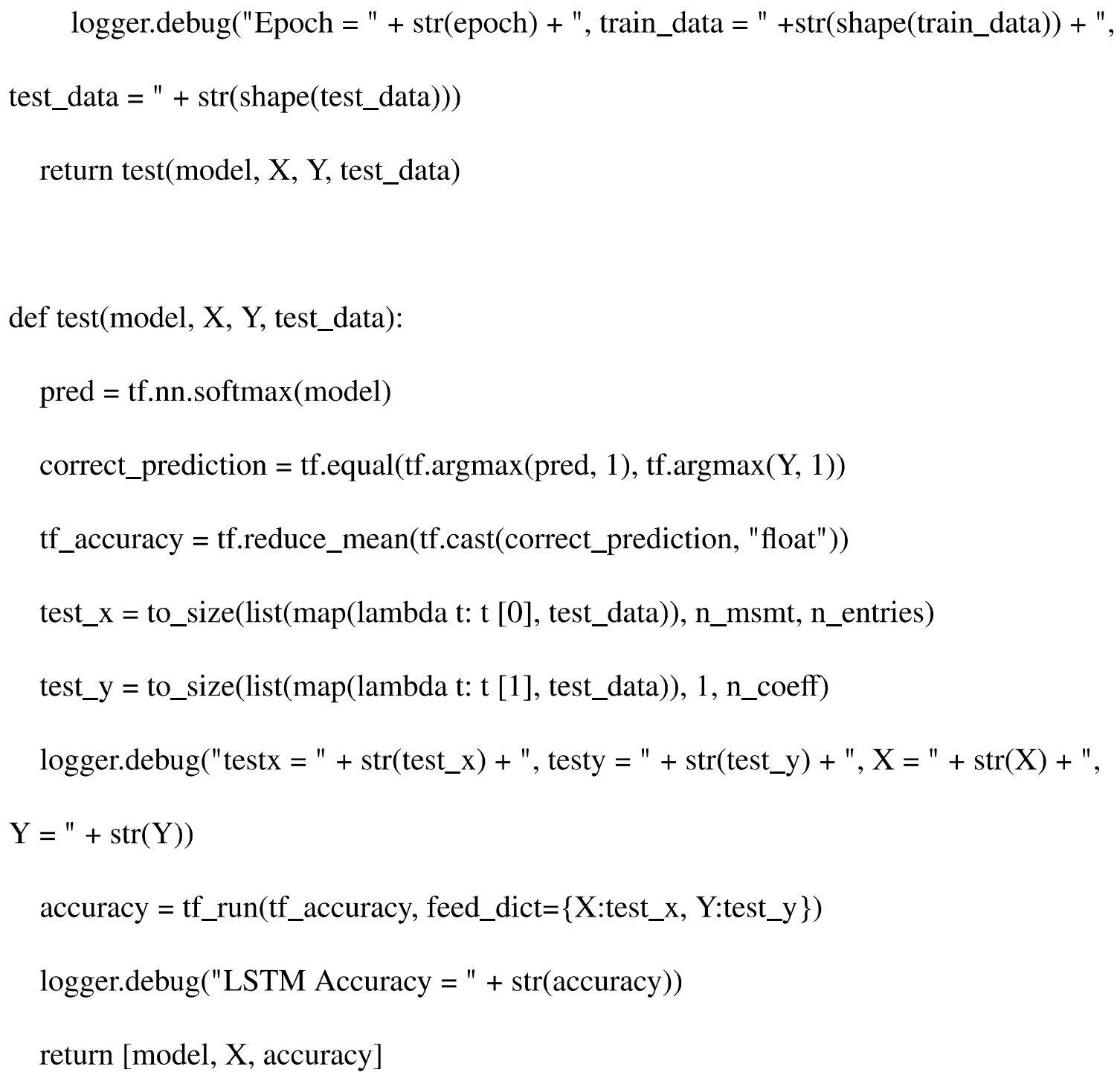

\section{Appendix C Generative Models For LQN Graphs}

Learning deep generative models for graphs [85] is a promising approach to automating creation of application system LQN models. This approach involves learning generative models over graph data, capturing both structural and attribute information about those 
graphs. The ability to generate graph samples of arbitrary graphs in many domains such as synthetic and real molecular graphs is useful in generating LQN models.

This approach uses graph neural networks to express probabilistic dependencies between the graph model nodes and edges, and can learn distributions over any arbitrary graph. Like other algorithms being utilized, independence from graph size and invariance to isomorphism are important qualities. Similar work has been done using generative graphs as intermediate representations in reasoning tasks [87]. That approach makes stronger assumptions about the number of nodes per sentence, and intermediate probabilities between edges, that make it less generalized than this deep generative model approach [85] which makes no such assumptions.

\section{C.1 Sequential Graph Generation Process For LQN Models}

The main steps of the process involve creation of a node, and then connection of this node to one or more existing graph nodes by creating one or more edges, one at a time. The steps involved in this process are

1. From sampling, decide whether to add a node of a certain node type, or to terminate

2. Check if new edges are needed on this added node 
3. Select an existing graph node and add an edge from it to the new node

4. Go to step 2) and repeat until no new edges are added

5. Go to step 1) and repeat until termination

The decisions to add a node, add an edge and select a node are provided by three

functions $f_{\text {addnode }}, f_{\text {addedge }}$ and $f_{\text {nodes }}$. These functions are also called modules since they usually represent neural networks. The graph generation process can then be described as a sequence of calls to these functions. Once defined that way, other sequence learning models like LSTMs and language models can be used to model it. Graph nets are used here because the information propagation steps are more sensitive to the underlying structure of that sequence.

\section{C.2 Training Graph Generation Models For LQN Graphs}

Structure building decisions produced by the above modules are governed by the following equations (Section 4.1 in [87]):

$$
\begin{aligned}
& \mathrm{h}^{(\mathrm{T})}{ }_{\mathrm{V}}=\operatorname{prop}^{(\mathrm{T})}\left(\mathrm{h}_{\mathrm{V}}, \mathrm{G}\right) \\
& \mathrm{h}_{\mathrm{G}}=\mathrm{R}\left(\mathrm{h}^{(\mathrm{T})}{ }_{\mathrm{V}}, \mathrm{G}\right) \\
& f_{\text {addnode }}(\mathrm{G})=\operatorname{softmax}\left(f_{\text {an }}\left(\mathrm{h}_{\mathrm{G}}\right)\right) \\
& f_{\text {addedge }}(\mathrm{G}, \mathrm{v})=\sigma\left(f_{\text {ae }}\left(\mathrm{h}_{\mathrm{G}}, \mathrm{h}^{(\mathrm{T})}{ }_{\mathrm{V}}\right)\right) \\
& \mathrm{s}_{\mathrm{U}}=\mathrm{f}_{\mathrm{S}}\left(\mathrm{h}^{(\mathrm{T})}{ }_{\mathrm{U}}, \mathrm{h}^{(\mathrm{T})}{ }_{\mathrm{v}}\right), \forall \mathrm{u} \in \mathrm{V} \\
& f_{\text {nodes }}(\mathrm{G}, \mathrm{v})=\operatorname{softmax}(\mathrm{s})
\end{aligned}
$$


Where $\mathrm{G}=(\mathrm{V}, \mathrm{E})$ is the training graph, $\mathrm{h}_{\mathrm{V}}$ is the set node embeddings, prop () represents a single step propagation, $\mathrm{h}_{\mathrm{V}}{ }^{(\mathrm{T})}$ is the node embedding after propagation step $\mathrm{T}$ (eq (5) to (10) in [87]).

For use in LQN model creation, the main issue is training data. To train the graph net, an iterative approach is useful. Given the application to be modelled, and known LQN model parameters like client request arrival rates, numbers of application services and instances, resources like databases and processors, request times and task demands. A sufficiently large dataset of parameters from the target application is collected and encoded into a graph embedding. This embedding is to be used in the generation process as a Conditional Generative Model (Section 4.1 in [87]).

$$
h_{\mathrm{V}}=\mathrm{f}_{\text {init }}\left(\mathrm{R}_{\text {init }}\left(\mathrm{h}_{\mathrm{V}}, \mathrm{G}\right), \mathrm{x}_{\mathrm{V}}\right)
$$

The above operation, node embedding initialization, is a popular way of doing the conditional generative model operation, with $\mathrm{x}_{\mathrm{V}}$ as the node conditioning information.

\footnotetext{
Also, sample LQN graphs need to be created for training the generative model. This training dataset can be created using a set of known LQN models covering known design patterns, focussing on the target application's domain. These models would represent key components of the system (LQN tasks and entries). Popular target system architectures are then drawn up programmatically combining these components to create a training data set. Normal training is done with the conditional generative model approach using the collected application data periodically in $\mathrm{f}_{\text {init }}$.
} 
N.B: Deep generative graph models for LQN has been partially implemented as part of this thesis. However, it is considered non-essential to the overall design of the controller, and hence due to time and scope constraints only the partial implementation and proposal are provided at this point. 


\section{References}

[1] Project page on Github with source code, readme \& system requirements: https://github.com/mikdangana//stm_ekf/, last seen: 8-Nov-2019

[2] Extended Kalman Filter python library: https://filterpy.readthedocs.io/en/latest/kalman/ExtendedKalmanFilter.html, last seen: 8-Nov-2019

[3] Burr Settles, "Synthesis Lectures on Artificial Intelligence and Machine Learning" University of Wisconsin-Madison, in Computer Sciences Technical Report volume 6 issue 1, 30-June-2012

[4] R. van der Merwe. "Sigma-Point Kalman Filters for Probabilistic Inference in Dynamic State-Space Models.” PhD Thesis, OGI School of Science \& Engineering, Oregon Health \& Science University, 2004

[5] D. A. Terejanu, "Extended Kalman Filter Tutorial", Department of Computer Science and Engineering, University of Buffalo, 2009

[6] K. Greef, "LSTM: A Search Space Odyssey", IEEE Transactions on Neural Networks And Learning Systems, Volume 28 Issue 10, October 2017

[7] Goodfellow, Bengio, Courville, “Deep Learning”, MIT Press, 2015, pg 184

[8] Simon Haykin, "Kalman Filtering And Neural Networks", Wiley \& Sons, Communications Research Lab, McMaster University, Hamilton ON CA, 2001

[9] Cornel Barna, Hamzeh Khazaei, Marios Fokaefs and Marin Litoiu, "Delivering Elastic Containerized Cloud Applications To Enable Devops", York University, 
ON CA, Conference: "Proceedings of the 12th International Symposium On Software Engineering and Adaptive Self-Management Systems", IEEE Press Volume 65-75

[10] Raul G Krishan, Uri Shalit, David Sontag, "Structured Inference Networks for Nonlinear State Space Models", Courant Institute of Mathematical Sciences, New York University, Thirty First AAAI Conference on Artificial Intelligence Feb 2017

[11] Leslie N Smith, "Cyclical Learning Rates for Training Neural Networks", IEEE Winter Conference on Applications of Computer Vision”, US Naval Research Laboratory, Optical Sciences Division, Washington USA, 2017

[12] R. O. Duda, P. E. Hart and D. G. Stork, "Pattern Classification”, 2nd Edition, John Wiley \& Sons, 2000

[13] Jim Warner, Top Command Manual Page, Ubuntu Organization

[14] Klaus Greff; Rupesh Kumar Srivastava; Jan Koutník; Bas R. Steunebrink; Jürgen Schmidhuber (2015). "LSTM: A Search Space Odyssey". IEEE Transactions on Neural Networks and Learning Systems. 28 (10).

[15] Greg Franks, Peter Maly, Murray Woodside, Dorina C. Petriu, and Alex Hubbard, "Layered Queueing Network Solver and Simulator User Manual". Real-time and Distributed Systems Lab, Carleton University, Ottawa http://www.sce.carleton.ca/rads/lqns/LQNSUserMan.pdf, last seen: 8-Nov-2019 
[16] Tao Zheng, C. Murray Woodside, Marin Litoiu, "Performance Model

Estimation and Tracking Using Optimal Filters", IEEE Transactions on Software

Engineering, Volume 34, Issue 3, May-June 2008.

[17] Huseyin Coskun, Felix Achilles, Robert DiPietro, Nassir Navab1, Federico

Tombari, "Long Short-Term Memory Kalman Filters: Recurrent Neural

Estimators for Pose Regularization", arXiv:1708.01885v1 [cs.CV], ICCV, 6 Aug 2017

[18] A. Krull, F. Michel, E. Brachmann, S. Gumhold, S. Ihrke, and C. Rother, “6-DOF Model Based Tracking Via Object Coordinate Regression”, In Proc. Asian Conference on Computer Vision (ACCV), Nov 2014

[19] David Martens, Bart Baesens, Tony Gestel, Jan Vanthienen, "Comprehensible Credit Scoring Models Using Rule Extraction From Support Vector Machines”, European Journal Of Operational Research, Volume 183, Issue 3, 16 December 2007

[20] S. Salti and L. Di Stefano, "Online support vector regression of the transition model for the Kalman filter", Image and Vision Computing, 2012

[21] Y. Du, Y. Wong, Y. Liu, F. Han, Y. Gui, Z. Wang, M. Kankanhalli, and W. Geng, "Marker-less 3d human motion capture with monocular image sequence and height-maps” In European Conference on Computer Vision, pages 20-36. Springer, 2016

[22] Hosseinyalamdary S, “Deep Kalman Filter: Simultaneous Multi-Sensor Integration and Modelling; A GNSS/IMU Case Study", Department of Earth 
Observation Science (EOS), Faculty of Geo-information Science and Earth Observation (ITC), University of Twente, Enschede 7514AE, The Netherlands, 24 April 2018

[23] Tolga Ergen, Suleyman Serdar Kozat, "Efficient Online Learning Algorithms Based on LSTM Neural Networks", IEEE Transactions on Neural Networks and Learning Systems, Volume 29 Issue 8, August 2018 D. F. Specht, “A general regression neural network,” IEEE Trans. Neural Netw., vol. 2, no. 6, pp. 568-576, Nov. 1991.

[25] N. D. Vanli, M. O. Sayin, I. Delibalta, and S. S. Kozat, "Sequential nonlinear learning for distributed multiagent systems via extreme learning machines," IEEE Trans. Neural Netw. Learn. Syst., vol. 28, no. 3, pp. 546-558, Mar. 2017.

[26] J. Schmidhuber, "Deep learning in neural networks: An overview," Neural Netw., vol. 61, pp. 85-117, Available: http://www.sciencedirect.com/science/article/pii/S0893608014002135, Jan 2015

[27] M. Hermans and B. Schrauwen, "Training and analysing deep recurrent neural networks," in Proc. Adv. Neural Inf. Process. Syst., 2013, pp. 190-198.

[28] J. Mazumdar and R. G. Harley, "Recurrent neural networks trained with backpropagation through time algorithm to estimate nonlinear load harmonic currents,” IEEE Trans. Ind. Electron., vol. 55, no. 9, pp. 3484-3491, Sep. 2008

[29] J. A. Pérez-Ortiz, F. A. Gers, D. Eck, and J. Schmidhuber, "Kalman filters improve LSTM network performance in problems unsolvable by traditional recurrent nets," Neural Netw., vol. 16, no. 2, pp. 241-250, Mar. 2003. 
[30] F. A. Gers, J. A. Péerez-Ortiz, D. Eck, and J. Schmidhuber, "DEKF-LSTM,” in Proc. ESANN, pp. 369-376. 2002

[31] Y. N. Dauphin, R. Pascanu, C. Gulcehre, K. Cho, S. Ganguli, and Y. Bengio, "Identifying and attacking the saddle point problem in high dimensional non-convex optimization,” in Proc. 27th Int. Conf. Neural Inf. Process. Syst. (NIPS), Cambridge, MA, USA, pp. 2933-2941. Available: http://dl.acm.org/citation.cfm?id=2969033.2969154, 2014

[32] Y. Bengio, P. Simard, and P. Frasconi, "Learning long-term dependencies with gradient descent is difficult," IEEE Trans. Neural Netw., vol. 5, no. 2, pp. 157-166, Mar. 1994.

D. Iter, J. Kuck, P. Zhuang, "Target Tracking with Kalman Filtering, KNN and LSTMs", Stanford University, Dec. 2016

[34] Simo S“"arkk“a, Aki Vehtari, and Jouko Lampinen, "Rao-blackwellized particle filter for multiple target tracking", Information Fusion, 8(1):2-15, 2007

[35] Ju Hong Yoon, Chang-Ryeol Lee, Ming-Hsuan Yang, and Kuk-Jin Yoon, “Online multi-object tracking via structural constraint event aggregation", In Proceedings of the IEEE Conference on Computer Vision and Pattern Recognition, pages 1392-1400, 2016

[36] Shaofei Wang and Charless Fowlkes, "Learning optimal parameters for multi-target tracking”, In British Machine Vision Conference, 2015. 
[37] C. Ma, A. Wang, G. Chen, C. Xu, "Hand joints-based gesture recognition for noisy dataset using nested interval unscented Kalman filter with LSTM network", Springer-Verlag GmbH Germany, Springer Nature 11 May 2018

[38] Chen, F.C., Appendino, S., Battezzato, A., "Constraint study for hand exoskeleton”, Human Hand Kinematics And Dynamics. J.Robot. 2013, 3 (2013)

[39] A. Sallab, M. Abdou, E. Perot, S. Yogamani, "Deep Reinforcement Learning Framework for Autonomous Driving”, Society for Imaging Science and Technology, 2017

[40] R. Zhao, R. Yan, J. Wang, K. Mao, "Learning to Monitor Machine Health with Convolutional Bi-Directional LSTM Networks”, Sensors MDPI, 30 Jan 2017

[41] Ke, W.; Wu, L. "Mobile Location with NLOS Identification and Mitigation Based on Modified Kalman Filtering”, Sensors 2011, 11, 1641-1656.

[42] Taborri J., Scalona E., Palermo E., Rossi S., Cappa P., "Validation of Inter-Subject Training for Hidden Markov Models Applied to Gait Phase Detection in Children with Cerebral Palsy", Sensors 2015, 15, 24514-24529.

[43] C. Jiang, S. Chen, Y. Chen, B. Zhang, Z. Feng, H. Zhou, Y. Bo, “A MEMS IMU De-Noising Method Using Long Short Term Memory Recurrent Neural Networks (LSTM-RNN)”, Sensors MDPI, 15 Oct 2018

[44] Eling, C.; Klingbeil, L.; Kuhlmann, H. "Real-time single-frequency GPS/MEMS-IMU attitude determination of lightweight UAVs", Sensors 2015, 15, $26212-26235$. 
[45] Quinchia, A.G.; Falco, G.; Falletti, E.; Dovis, F.; Ferrer, C. "A comparison between different error modeling of MEMS applied to GPS/INS integrated systems”, Sensors 2013, 13, 9549-9588.

[46] R. Zhang, Q. Zou, "Time Series Prediction and Anomaly Detection of Light Curve Using LSTM Neural Network", IOP Conf. Series: Journal of Physics: Conf. Series 1061 (2018)

[47] Han Fei, Dong Hongli, Wang Zidong, "Improved Tobit Kalman filtering for systems with random parameters via conditional expectation,' Signal Processing, vol. 147, pp 35-45, 2018.

[48] H. Zen, Y. Agiomyrgiannakis, N. Egberts, F. Henderson, P. Szczepaniak, "Fast, compact, and High Quality LSTM-RNN Based Statistical Parametric Speech Synthesizers for Mobile Devices", Google arXiv:1606.06061v2 22 Jun 2016

[49] Zen, Heiga. "Statistical parametric speech synthesis: from HMM to LSTM-RNN", 10.13140/RG.2.1.4953.7045, 2015

[50] D. Hsu and S. Sabato, "Loss minimization and parameter estimation with heavy tails," arXiv:1307.1827, 2013.

[51] J.Tukey, "A survey of sampling from contaminated distributions," Contributions to probability and statistics, vol. 2, pp. 448-485, 1960.

[52] G. Henter, S. Ronanki, O. Watts, M. Wester, Z. Wu, and S. King, "Robust TTS duration modeling using DNNs," in Proc. ICASSP, pp. 5130-5134, 2016 
[53] K. Yu, T. Toda, M. G as’’1c, S. Keizer, F. Mairesse, B. Thomson, and S. Young, "Probablistic modelling of F0 in unvoiced regions in HMM based speech synthesis," in Proc. ICASSP, pp. 3773-3776, 2009

[54] V. Reddy, "NEON technology introduction,” ARM Corporation, 2008

[55] P. Malhotra, TV. Vishnu, A. Ramakrishnan, G. Anand, L. Vig, P. Agarwal, G. Shroff, "Multi-Sensor Prognostics using an Unsupervised Health Index based on LSTM Encoder-Decoder", TCS Research, arXiv:1608.06154v1, 22 Aug 2016

[56] E. Ramasso. "Investigating computational geometry for failure prognostics in presence of imprecise health indicator: Results and comparisons on c-mapss datasets", In 2nd European Conference of the Prognostics and Health Management Society., 2014

[57] P. Wang and G. Vachtsevanos. "Fault prognostics using dynamic wavelet neural networks", AI EDAM, 2001.

[58] K. Cho, B. Van Merrienboer, C. Gulcehre, D. Bahdanau, F. Bougares, H. Schwenk, and Y. Bengio. "Learning phrase representations using rnn encoder-decoder for statistical machine translation", arXiv preprint arXiv:1406.1078, 2014.

[59] I. Sutskever, O. Vinyals, and Q. V. Le. "Sequence to sequence learning with neural networks". arXiv:1409.3215 [cs.CL]. 2014.

[60] A. Saxena, K. Goebel, D. Simon, and N. Eklund. "Damage propagation modeling for aircraft engine run-to-failure simulation", In Prognostics and Health 
Management, 2008. PHM 2008. International Conference on, pages 1-9. IEEE, 2008.

[61] A. Agogino and K. Goebel. "Milling Data Set". BEST lab, UC Berkeley, 2007.

[62] Y. Liu, Y. Wang, X. Yang, L. Zhang, "Short-term Travel Time Prediction by Deep Learning: A Comparison of Different LSTM-DNN Models", Tongji University, IEEE 20th International Conference on Intelligent Transportation Systems (ITSC) 2017

[63] D. Billings and J. S Yang, "Application of the ARIMA Models to Urban Roadway Travel Time Prediction - A Case Study," IEEE Int. Conf. on Systems, Man and Cybernetics, pp 2529-2534, ARIMA, 2006

[64] M. S. Ahmed and A. R Cook, "Analysis of freeway traffic time-series data by using Box-Jenkins techniques", no. 722, ARIMA, 1979

[65] B. Pan, U. Demiryurek, and C. Shahabi, "Utilizing Real-World Transportation Data for Accurate Traffic Prediction," IEEE 12th Int. Conf. on Data Mining, pp. 595-604. H+ARIMA, 2012

[66] P. Duan, G. Mao, C. Zhang, and S. Wang, "STARIMA-based traffic prediction with time-varying lags," IEEE 19th Int. Conf. on Intelligent Transportation Systems (ITSC), 2016, pp. 1610-1615. STARIMA

[67] J. Howarth, J. Shawe-Taylor, T. Cheng, and J. Wang, "Local online kernel Ridge regression for forecasting of urban travel times," Transportation Research Part C: Emerging Technologies, vol. 46, pp. 151-178, 2014 
[68] W. Zheng, D.H. Lee, Q. Shi, "Short-term freeway traffic flow prediction: Bayesian combined neural network approach," J. Transportation Eng, vol 132, pp. 114-121, 2006.

[69] C. H. Wu, J. Ho, D. Lee, "Travel-time prediction with support vector regression," IEEE Trans. Intell. Transp. Syst., vol. 5, pp. 276-281, 2004

[70] G. E. Hinton, "Reducing the Dimensionality of Data with Neural Networks," Science, vol. 313, pp. 504-507, 2006

[71] W. Huang, G. Song, H. Hong, K. Xie, "Deep Architecture for Traffic Flow Prediction: Deep Belief Networks With Multitask Learning," IEEE Trans. Intell. Transp. Syst., vol 15, pp. 2191-2201, Oct 2014

[72] J. Li, S. Roy, J. Feng, T. Sim, "Happiness Level Prediction with Sequential Inputs via Multiple Regressions", ICMP 16, 12-16, Tokyo, ACM.978-1-4503-4556-9/16/11, November 2016

[73] A. Dhall, R. Goecke, and T. Gedeon. "Automatic group happiness intensity analysis”, IEEE Transactions on Affective Computing, 6(1):13-26, 2015.

[74] X. Huang, A. Dhall, G. Zhao, R. Goecke, and M. Pietikainen. "Riesz-based volume local binary pattern and a novel group expression model for group happiness intensity analysis", In BMVC, pages 34-1, 2015

[75] W. Mou, O. Celiktutan, and H. Gunes. "Group-level arousal and valence recognition in static images: Face, body and context", In Automatic Face and Gesture Recognition (FG), 2015 11th IEEE International Conference and Workshops on, volume 5, pages 1-6. IEEE, 2015. 
[76] D. Abhinav, G. Roland, J. Jyoti, H. Jesse, and G. Tom. "Video and group-level emotion recognition challenges", In 18th ACM International Conference on Multimodal Interaction, 2016

[77] A. Gias, G. Casale, M. Woodside, "ATOM: Model-Driven Autoscaling for Microservices", IEEE International Conference on Distributed Computing Systems (ICDCS), April 2019

[78] N. Kratzke and P.-C. Quint, "Understanding cloud-native applications after 10 years of cloud computing-a systematic mapping study," Journal of Systems and Software, vol. 126, pp. 1-16, 2017.

[79] T. Chen and R. Bahsoon, "Self-adaptive trade-off decision making for autoscaling cloud-based services," IEEE Transactions on Services Computing, vol. 10 , no. 4 , pp. $618-632,2017$

[80] C. Barna, M. Litoiu, M. Fokaefs, M. Shtern, and J. Wigglesworth, "Runtime performance management for cloud applications with adaptive controllers," in Proceedings of the 9th International Conference on Performance Engineering. ACM, pp. 176-183 2018

[81] M. Woodside, J. E. Neilson, D. C. Petriu, and S. Majumdar, "The stochastic rendezvous network model for performance of synchronous client-server-like distributed software," IEEE Transactions on Computers, vol. 44, no. 1, pp. 20-34, 1995. 
[82] Raj Jain, “Art of Computer Systems Performance Analysis Techniques For Experimental Design Measurements Simulation And Modelling”, Wiley Computer Publishing, ISBN 0471503363, 1991

[83] SPEC Cloud IaaS 2018 Benchmark, https://www.spec.org/cloud_iaas2018/, version 1.0, December 2018

[84] Amazon Web Services "EC2 Virtual Machine Instance Types" https://aws.amazon.com/ec2/instance-types/, last seen: 8-Nov-2019

[85] Yujia Li, Oriol Vinyals, Chris Dyer, Razvan Pascanu, Peter Battaglia, "Deep Generative Models Of Graphs”, arXiv:1803.03324v1 [cs.LG] 8 Mar 2018

[86] Henaff, Mikael, Bruna, Joan, and LeCun, Yann. "Deep Convolutional Networks On Graph-structured Data” arXiv:1506.05163, 2015

[87] Johnson, Daniel D. “Learning Graphical State Transitions”. In International Conference on Representation Learning (ICLR), 2017.

[88] Moore, Bruce., "Principal Component Analysis in Linear Systems: Controllability, Observability \& Model Reduction”, IEEE Transactions On Automatic Control, Vol. Ac-26, No. 1, February 1981.

[89] R. E. Kalman, "Irreducible realizations and the degree of a rational matrix", J. Applied Math., Vol. 13, No. 2, pp. 520-544, 1965.

[90] U. Zulfiqar, M. Imran, A. Ghafoor, “Cross-Gramian based frequency-weighted model order reduction technique”, Electronic Letters, Vol. 52, No. 16, 2016 
[91] Xingfu Zhang, Xiangmin Ren, “Two Dimensional Principal Component Analysis based Independent Component Analysis for face recognition", International Conference on Multimedia Technology, July 2011

[92] M. Turk and A. Pentland, "Eigenfaces for recognition," Journal Of Cognitive Neuroscience, Vol. 3, pp. 71-86. 1991

[93] Richard H Middleton, Graham C. Goodwin, "Adaptive control of time-varying linear systems", IEEE Transactions on Automatic Control, Volume: 33 , Issue: 2 , Feb 1988

[94] "Database of Faces", https://www.cl.cam.ac.uk/research/dtg/attarchive/facedatabase.html, Cambridge University Engineering Department, last seen: 8-Nov-2019

[95] XiaoGuang Feng, P. Milanfar, "Multiscale Principal Components Analysis For Local Image Orientation Estimation", Conference Record of the Thirty-Sixth Asilomar Conference on Signals, Systems and Computers, 2002

[96] S. Borguet, O. Leonard, "Coupling principal component analysis and Kalman filtering algorithms for on-line aircraft engine diagnostics", Control Engineering Practice 17, 2009

[97] Haykin, S, "Kalman filtering and neural networks. Wiley series on adaptive and learning systems for signal processing, communications and control", New York, Wiley, 2001 
[98] Rico-Sulayes, Antonio. "Reducing Vector Space Dimensionality in Automatic Classification for Authorship Attribution". Revista Ingeniería Electrónica, Automática y Comunicaciones. 38 (3): 26-35, 2017

[99] Gross, Donald; Carl M. Harris, "Fundamentals of Queueing Theory" Wiley. ISBN 978-0-471-32812-4, 1998

[100] Jolliffe I.T. “Principal Component Analysis”, Series: Springer Series in Statistics, 2nd ed., Springer, NY, XXIX, 487 p. 28 illus. ISBN $978-0-387-95442-4,2002$

[101] B. J. Odelson, M. R. Rajamani, J. B. Rawlings, "A new autocovariance least-squares method for estimating noise covariances", Automatica, vol. 42, no. 2, pp. 303-308, Feb. 2005.

[102] M. Woodside, T. Zheng, M. Litoiu, "The Use of Optimal Filters to Track Parameters of Performance Models”, Proc. 2nd Int Conf on Quantitative Evaluation of Systems (QEST05)”, Torino, Italy, 2005

[103] Yixin Diao, Xue Lui, Steve Froehlich, Joseph L Hellerstein, Sujay Parekh, and Lui Sha. “On-Line Response Time Optimization of An Apache Web Server,” Int. Workshop on Quality of Service, 2003

[104] D. A. Menasce, M. Bennani, "On the Use of Performance Models to Design Self-Managing Computer Systems," Computer Measurement Group Conference, Dallas, 2003 
[105] Litoiu M., Woodside M., Zheng T., "Hierarchical model based autonomic control of software systems," Proc. of Design and Evolution of Autonomic Software Workshop, St. Louis, USA, 2005

[106] D. A. Menasce, M. Bennani, "On the Use of Performance Models to Design Self-Managing Computer Systems," Proc. 2003 Computer Measurement Group Conference, Dallas, Dec, 2003

[107] Morgan Kaufmann, "Usability Engineering”, San Francisco, ISBN 0-12-518406-9, 1993

[108] Sebastian Ruder, "An overview of gradient descent optimization algorithms", arXiv preprint arXiv:1609.04747, 2016

[109] Justin Bayer, Daan Wierstra, Julian Togelius and J"urgen Schmidhuber, "Evolving memory cell structures for sequence learning". IDSIA, Galleria 2, 6928 Manno-Lugano, Switzerland, 2009

[110] Tensorflow 2.0, www.tensorflow.org, Google Inc, 2019

[111] Yanhong Zhou, Shukai Cao, Dong Wen, Huiyang Zhang, Liqiang Zhao, “The study of face recognition based on hybrid Principal Component Analysis and Independent Component Analysis", International Conference on Electronics, Communication and Control (ICECC), Sept 2011

[112] Nilabja Roy, Abhishek Dubey and Aniruddha Gokhale, "Efficient Autoscaling in the Cloud using Predictive Models for Workload Forecasting", University Of Vanderbilt, Nashville, IEEE 4th International Conference on Cloud Computing, 2011 
[113] B. Urgaonkar, P. Shenoy, A. Chandra, P. Goyal, and T. Wood, “Agile dynamic provisioning of multi-tier internet applications," ACM Trans. Auton. Adapt. Syst., vol. 3 , no. 1 , pp.1-39, 2008

[114] P. Padala, K. Shin, X. Zhu, M. Uysal, Z. Wang, S. Singhal, A. Merchant, and K. Salem, "Adaptive control of virtualized resources in utility computing environments," ACM SIGOPS Operating Systems Review, vol. 41, no. 3, p. 302, 2007.

[115] Zhiheng Huang, Wei Xu, Kai Yu, "Bidirectional LSTM-CRF Models for Sequence Tagging", arXiv:1508.01991,pg. 2, August 2015 